\title{
Conversion of Cyclic Vinyl Sulfones to Transposed Vinyl Phosphonates
}

\author{
Mohammad N. Noshi, Ahmad El-awa, Eduardo Torres and Philip L. Fuchs* \\ Department of Chemistry, Purdue University, West Lafayette,IN, 47907 \\ pfuchs@purdue.edu
}

\section{Supporting Information}

\section{Table of Contents}

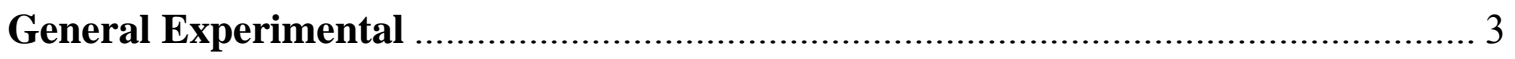

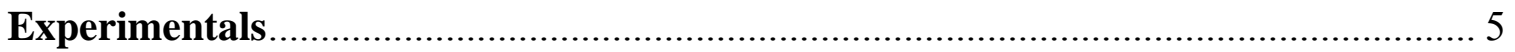

Preparation of cis-hydroxyepoxide 8a:

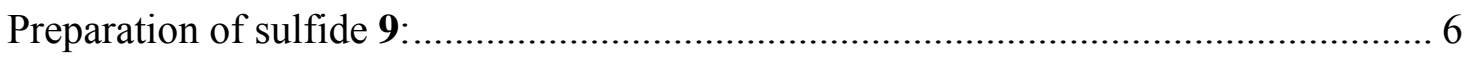

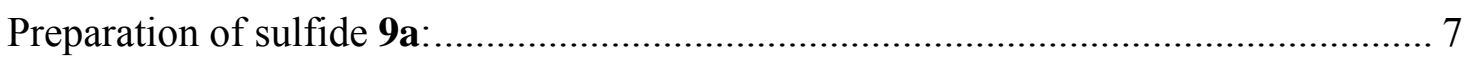

Preparation of sulfide 9a from epoxide 8a (one pot procedure):.................................. 8

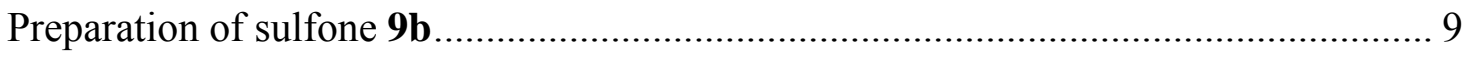

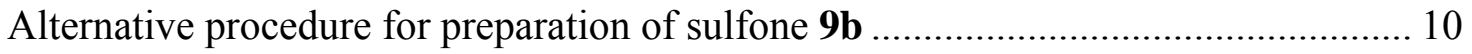

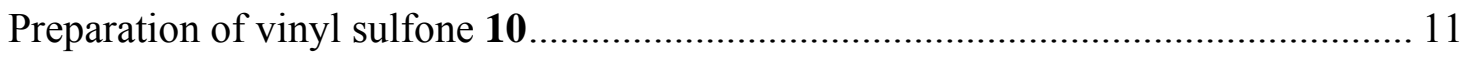

Preparation of aldehyde ester 11 ........................................................................ 12

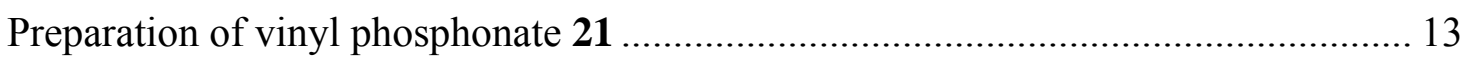

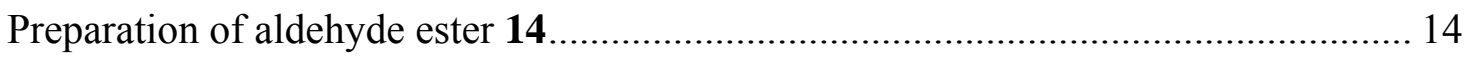

Preparation of phosphonate sulfone adduct 22 ........................................................... 15

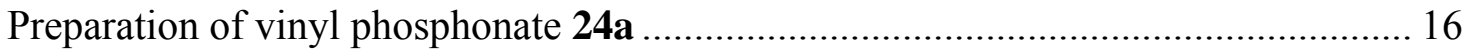

Preparation of vinyl phosphonate 25a ...................................................................... 17 
Preparation of vinyl phosphonate 18a ................................................................. 18

Characterization of dienyl phosphonate 27b ....................................................... 19

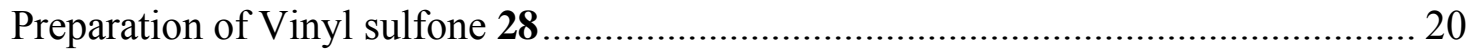

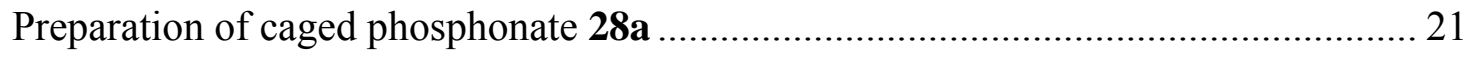

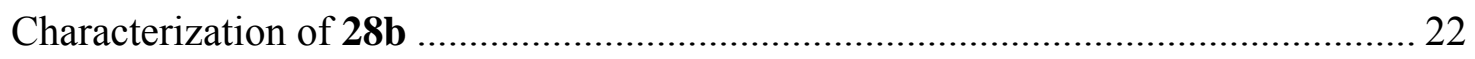

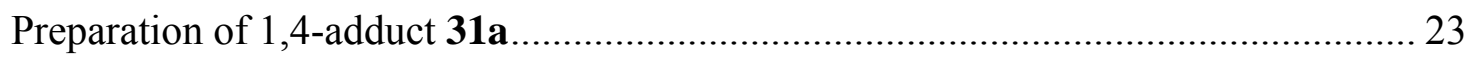

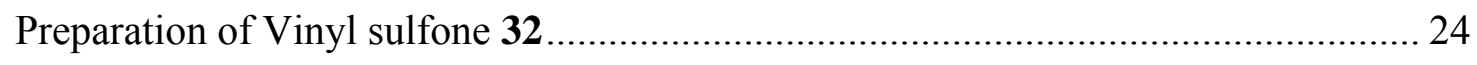

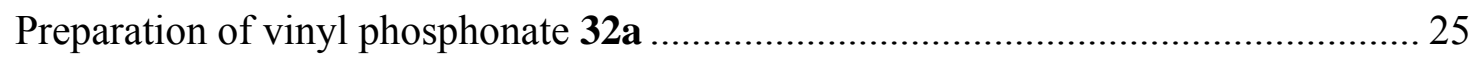

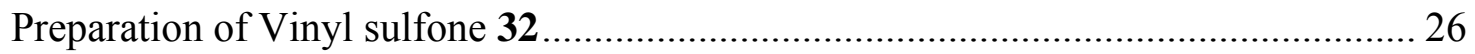

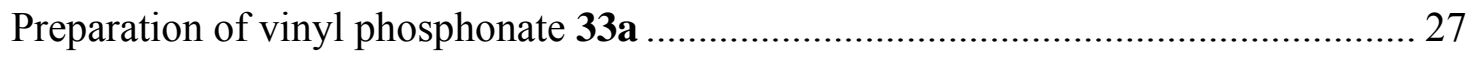

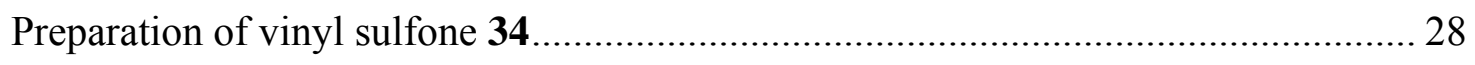

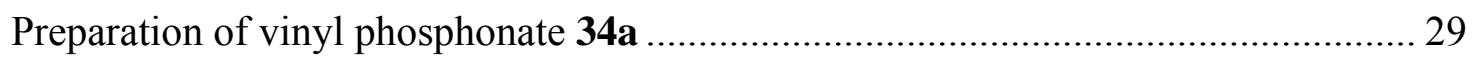

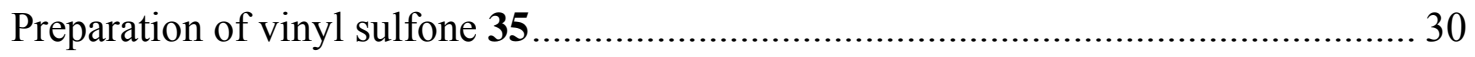

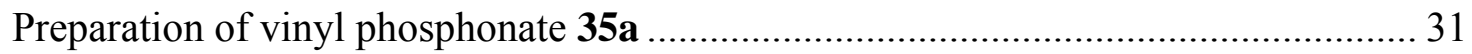

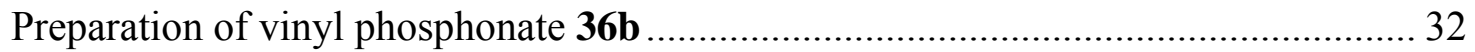

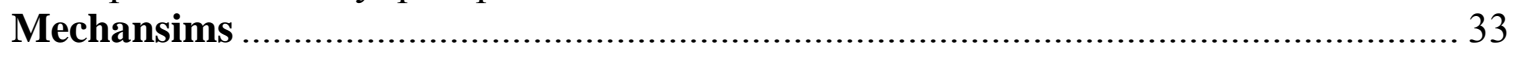

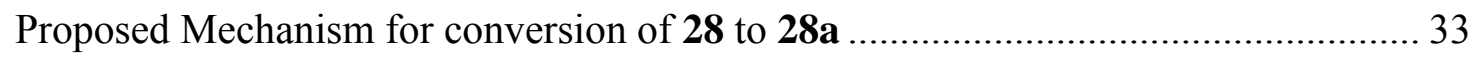

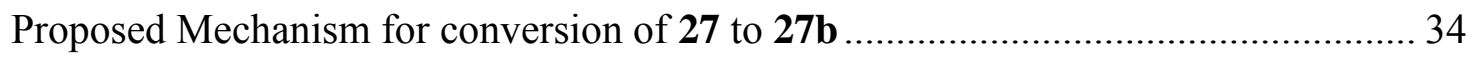




\section{General Experimental}

All reactions were performed in oven-dried $\left(140^{\circ} \mathrm{C}\right)$ or flame dried glassware under a positive pressure of dry $\mathrm{N}_{2}$. All dry solvents were freshly distilled and were withdrawn with oven-dried glass syringes $\left(140^{\circ} \mathrm{C}\right.$, overnight) and the syringes were purged with dry $\mathrm{N}_{2}$ three times just before use. All temperatures refer to bath temperatures unless otherwise indicated. All purchased materials were used without further purification. All yields are based on pure and thoroughly dried starting materials and products. Tetrahydrofuran (THF) and diethyl ether were distilled from benzophenone ketyl. Methylene chloride $\left(\mathrm{CH}_{2} \mathrm{Cl}_{2}\right)$ was distilled from calcium hydride. Methanol was distilled from magnesium turnings/catalytic iodine or purchased spectra-grade. Sodium sulfate $\left(\mathrm{Na}_{2} \mathrm{SO}_{4}\right)$ was anhydrous. All reaction, workup, chromatographic and recrystallization solvents were distilled. Unless otherwise indicated, all reactions were performed under a positive atmosphere of dry $\mathrm{N}_{2}$ or argon and reaction flasks were fitted with rubber septas for the introduction of substrates/reagents via syringe. Reaction progress was monitored by thin layer chromatography (TLC) using silica gel $60 \mathrm{~F}-254$ plates (EM reagents, $0.25 \mathrm{~mm}$ ). The TLC plates were visualized with a UV lamp (254 nm) and/or with TLC staining solutions activated with heat. The most commonly employed TLC staining solutions were: (i) p-anisaldehyde stain (PAA) $(1350 \mathrm{~mL}$ absolute ethanol, $50 \mathrm{~mL}$ concentrated $\mathrm{H}_{2} \mathrm{SO}_{4}, 37 \mathrm{~mL}$ p-anisaldehyde), (ii) $\mathrm{KMnO}_{4}$ stain (1.5 g

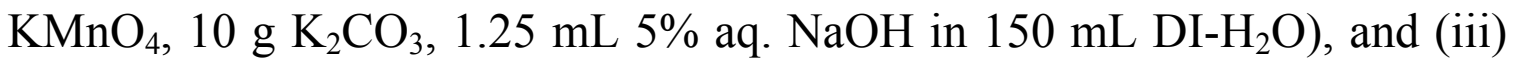
O-vanillin stain $\left(2 \mathrm{~g}\right.$ o-vanillin, $10 \mathrm{~mL} \mathrm{H}_{2} \mathrm{SO}_{4}, 45 \mathrm{~mL} \mathrm{MeOH}, 45 \mathrm{~mL}$ DI$\mathrm{H}_{2} \mathrm{O}$ ). 
${ }^{1} \mathrm{H}$ NMR, ${ }^{13} \mathrm{C}$ NMR and ${ }^{31} \mathrm{P}$ NMR were recorded on Varian Inova $300(300$ $\left.\mathrm{MHz},{ }^{1} \mathrm{H} ; 75 \mathrm{MHz},{ }^{13} \mathrm{C} ; 282.216 \mathrm{MHz},{ }^{31} \mathrm{P}\right)$ machine. NMR spectra were determined in chloroform- $\mathrm{d}_{1}\left(\mathrm{CDCl}_{3}\right)$ and are reported in parts per million (ppm) from the residual chloroform $\left({ }^{1} \mathrm{HNMR}, 7.27 \mathrm{ppm}\right.$ and ${ }^{13} \mathrm{C} \mathrm{NMR}, 77.0$ ppm) standard. Peak multiplicities in ${ }^{1} \mathrm{H}$ NMR, when reported, are abbreviated as $\mathrm{s}$ (singlet), $\mathrm{d}$ (doublet), $\mathrm{t}$ (triplet), q (quartet), $\mathrm{p}$ (pentet), $\mathrm{h}$ (hextet), m (multiplet) and/or ap (apparent) and/or br (broad). ${ }^{31} \mathrm{P}$ NMR was determined using an external $85 \% \mathrm{H}_{3} \mathrm{PO}_{4}$ standard; $\delta 0$ ppm. Coupling constants, $J$, are reported in $\mathrm{Hz}$ (Hertz). ${ }^{1} \mathrm{H}$ NMR values, when reported, are approximated to two decimal places while ${ }^{13} \mathrm{C}$ NMR and $J$ values are approximated to one decimal place. Melting points were obtained on a MEL-TEMP capillary melting point apparatus or MP A100 optimelt automated melting point system and are uncorrected. Mass spectra were run by the Purdue University campus wide mass spectrometry facility. 


\section{$\underline{\text { Experimentals }}$}

Preparation of cis-hydroxyepoxide 8a:

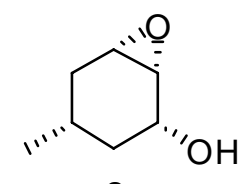

$8 \mathbf{a}$

A flame dried flask was charged with allylic alcohol 8 (1.0 g, $8.9 \mathrm{mmol})$, $\mathrm{NaHCO}_{3}(1.5 \mathrm{~g}, 18 \mathrm{mmol})$, and dry $\mathrm{CH}_{2} \mathrm{Cl}_{2}(25 \mathrm{~mL})$, and the mixture was cooled to $0^{\circ} \mathrm{C}$ under dry $\mathrm{N}_{2}$ atmosphere for 30 minutes. $70 \%$ MCPBA (1.97 $\mathrm{g}$, $8 \mathrm{mmol})$ was dissolved in dry $\mathrm{CH}_{2} \mathrm{Cl}_{2}(20 \mathrm{~mL})$ and the solution was cannulated slowly into the reaction flask. Stirring was continued for $2 \mathrm{~h}$ and the reaction mixture was allowed to reach ambient temperature. After reaction completion, the reaction mixture was cooled again to $0^{\circ} \mathrm{C}$, washed with aq. saturated $\mathrm{Na}_{2} \mathrm{SO}_{3}$ then with aq. saturated $\mathrm{K}_{2} \mathrm{CO}_{3}$ solution. All aq. extracts were combined then extracted with ether $(3 \times 45 \mathrm{~mL})$ and the organic extracts were added to the original $\mathrm{CH}_{2} \mathrm{Cl}_{2}$ layer. The organic layer was washed with brine, dried over anhydrous $\mathrm{Na}_{2} \mathrm{SO}_{4}$ then concentrated via rotary evaporation to afford the crude product as a faint yellow oil (19:1 de). Purification by flash column chromatography (20\% ethyl acetate/hexanes) afforded the desired epoxide as a colorless oil (1.01 g, 89\%). TLC (30\% ethyl acetate/hexanes) $R_{f} 0.16 ;{ }^{1} \mathrm{H}$ NMR $\left(\mathrm{CDCl}_{3}, 300 \mathrm{MHz}\right) \delta 3.90(\mathrm{dt}, J=$ $6.9,10.8 \mathrm{~Hz}, 1 \mathrm{H}), 3.20(\mathrm{q}, J=4.2 \mathrm{~Hz}, 1 \mathrm{H}), 3.18\left(\mathrm{~s}, 1 \mathrm{H}\right.$, exchange $\left.\mathrm{D}_{2} \mathrm{O}\right)$, $3.14(\mathrm{~d}, J=8.4 \mathrm{~Hz}, 1 \mathrm{H}), 1.80(\mathrm{dt}, J=5.6,15.8 \mathrm{~Hz}, 1 \mathrm{H}), 1.52$ (ddd, $J=1.1$, 2.3, $12.4 \mathrm{~Hz}, 1 \mathrm{H}), 1.36$ ( br. m, 1H), 1.24 (dd, $J=11.2,14.6 \mathrm{~Hz}, 1 \mathrm{H}), 1.15$ 
$(\mathrm{q}, J=11.8,1 \mathrm{H}), 0.78(\mathrm{~d}, J=5.6 \mathrm{~Hz}, 3 \mathrm{H}) ;{ }^{13} \mathrm{C} \mathrm{NMR}\left(\mathrm{CDCl}_{3}, 75 \mathrm{MHz}\right) \delta$ 68.9, 56.1, 54.9, 35.8, 31.2, 28.0, 22.1; LRMS (CI) calculated for $\mathrm{C}_{7} \mathrm{H}_{12} \mathrm{O}_{2}$ $(\mathrm{M}+\mathrm{H})$ 129.0916, found 129; HRMS (CI) calculated for $\mathrm{C}_{7} \mathrm{H}_{12} \mathrm{O}_{2}(\mathrm{M}+\mathrm{H}-$ $\left.\mathrm{H}_{2} \mathrm{O}\right) 111.0810$, found 111.0811 .

This procedure was scaled up using allylic alcohol 8 (10 g, $89 \mathrm{mmol})$ and yielded epoxide 8a as a yellow oil $(9.3 \mathrm{~g}, 82 \%){ }^{1}$

\section{Preparation of sulfide 9:}

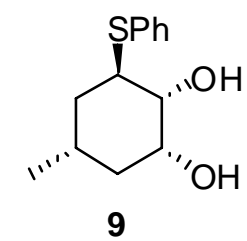

A flame dried flask was charged with $60 \% \mathrm{NaH}$ in oil $(0.58 \mathrm{~g}, 14.5 \mathrm{mmol})$ then with hexanes $(20 \mathrm{~mL})$ and the mixture was cooled to $0^{\circ} \mathrm{C}$ while stirring. Stirring was stopped then, after settling, hexanes was cannulated out and the process was repeated twice. A solution of PhSH (1.54 mL, $15 \mathrm{mmol})$ in THF $(25 \mathrm{~mL})$ was then cannulated slowly to the $\mathrm{NaH}$ suspension and stirring was continued for 30 minutes at $0^{\circ} \mathrm{C}$. This solution was then cannulated slowly to a cold solution of hydroxyepoxide 8a $(1.28 \mathrm{~g}, 10 \mathrm{mmol})$ in THF $(25 \mathrm{~mL})$ and the mixture was allowed to reach ambient temperature while stirring was continued for $3 \mathrm{~h}$. The reaction mixture was diluted with ether $(25 \mathrm{~mL})$ then ice chips were added slowly until a clear solution was produced. Brine (75 $\mathrm{mL}$ ) was added and the mixture was stirred for additional 30 minutes. The organic layer was separated, dried over $\mathrm{Na}_{2} \mathrm{SO}_{4}$ and then concentrated via

\footnotetext{
${ }^{1}$ The decreased yield upon scaling up is a consequence of the remarkable aqueous solubility of epoxide 8a and the relative difficulty to efficiently extract all aqueous phases.
} 
rotary evaporation to give 9 as a crude yellow oil (de 100\%). Purification with flash column chromatography (30\% ethyl acetate/hexanes) afforded sulfide 9 as a clear yellow oil that solidifies to give a yellow crystalline solid (2.19 g, 92\%). Melting range $88.9-90.8^{\circ} \mathrm{C}$. TLC (50\% ethyl acetate/hexanes) $R_{f} 0.40 ;{ }^{1} \mathrm{H} \mathrm{NMR}\left(\mathrm{CDCl}_{3}, 300 \mathrm{MHz}\right) \delta 7.40(\mathrm{~m}, 5 \mathrm{H}), 4.20$ (ddd, $J=3.0,4.2$, $11.1 \mathrm{~Hz}, 1 \mathrm{H}), 3.98$ (t, $J=3.0 \mathrm{~Hz}, 1 \mathrm{H}), 3.68$ (q, $J=3.3,1 \mathrm{H}), 3.32$ ( br. s, $1 \mathrm{H}$, exchange $\left.\mathrm{D}_{2} \mathrm{O}\right), 2.92$ (br. $\mathrm{s}, 1 \mathrm{H}$, exchange $\left.\mathrm{D}_{2} \mathrm{O}\right), 1.92(\mathrm{~m}, 1 \mathrm{H}), 1.82(\mathrm{dd}, J=$ 3.9, $13.5 \mathrm{~Hz}, 1 \mathrm{H}), 1.41$ (q, $J=11.9,1 \mathrm{H}), 1.02(\mathrm{~d}, J=6.2 \mathrm{~Hz}, 3 \mathrm{H}) ;{ }^{13} \mathrm{C} \mathrm{NMR}$ $\left(\mathrm{CDCl}_{3}, 75 \mathrm{MHz}\right) \delta 134.9,131.1,129.1,126.8,71.0,68.1,49.9,36.9,33.0$, 27.1, 21.9; LRMS (EI) calculated for $\mathrm{C}_{13} \mathrm{H}_{18} \mathrm{O}_{2} \mathrm{~S}(\mathrm{M}+)$ 238.1028, found 238; HRMS (EI) calculated for $\mathrm{C}_{13} \mathrm{H}_{18} \mathrm{O}_{2} \mathrm{~S}(\mathrm{M}+)$ 238.1028, found 238.1028.

Preparation of sulfide 9a:

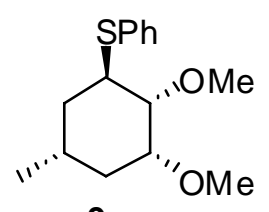

$9 a$

A solution of sulfide $9(1.9 \mathrm{~g}, 8 \mathrm{mmol})$ in THF $(40 \mathrm{~mL})$ was cooled to $0^{\circ} \mathrm{C}$ for 10 minutes then was treated with $\mathrm{MeI}(5.6 \mathrm{~mL}, 80 \mathrm{mmol})$ and stirring was continued for additional 10 minutes. $\mathrm{NaH}(0.72 \mathrm{~g}, 18 \mathrm{mmol})$ was added in portions and the reaction was allowed to reach ambient temperature while stirring was continued for $3 \mathrm{~h}$. After completion, ether $(20 \mathrm{~mL})$, brine $(60$ $\mathrm{mL}$ ) were added and the mixture was stirred for 15 minutes. Ice chips were added slowly until a clear two phase mixture was obtained. The organic layer was separated, dried over $\mathrm{Na}_{2} \mathrm{SO}_{4}$ and then was concentrated via rotary evaporation to afford a crude yellow oil. Purification with flash column chromatography (10\% ethyl acetate/hexanes) afforded the desired sulfide as 
a clear yellow oil (1.94 g, $91 \%)$. TLC (10\% ethyl acetate/hexanes) $R_{f} 0.24$;

${ }^{1} \mathrm{H}$ NMR $\left(\mathrm{CDCl}_{3}, 300 \mathrm{MHz}\right) \delta 7.40(\mathrm{~m}, 5 \mathrm{H}), 3.77(\mathrm{~m}, 1 \mathrm{H}), 3.72(\mathrm{~m}, 1 \mathrm{H})$, 3.65 (q, $J=2.4 \mathrm{~Hz}, 1 \mathrm{H}), 3.44$ (s, 3H), 3.42 (s, 3H), 1.90 (m, 1H), 1.85 (dd, $J$ $=1.5,12.9 \mathrm{~Hz}, 1 \mathrm{H}), 1.70(\mathrm{dt}, J=3.3,8.4 \mathrm{~Hz}, 1 \mathrm{H}), 1.40(\mathrm{q}, J=12.9 \mathrm{~Hz}, 1 \mathrm{H})$, $1.00(\mathrm{~d}, J=6.3 \mathrm{~Hz}, 3 \mathrm{H}) ;{ }^{13} \mathrm{C} \mathrm{NMR}\left(\mathrm{CDCl}_{3}, 75 \mathrm{MHz}\right) \delta 135.1,130.9,128.8$, 127.2, 78.1, 77.3, 57.5, 56, 46, 33.8, 33.3, 26.3, 21.8; LRMS (CI/EI) calculated for $\mathrm{C}_{15} \mathrm{H}_{22} \mathrm{O}_{2} \mathrm{~S}(\mathrm{M}+)$ 266.1341, found 266; HRMS (CI) calculated for $\mathrm{C}_{15} \mathrm{H}_{22} \mathrm{O}_{2} \mathrm{~S}(\mathrm{M}+)$ 266.1341, found 266.1340.

Preparation of sulfide 9a from epoxide 8a (one pot procedure):

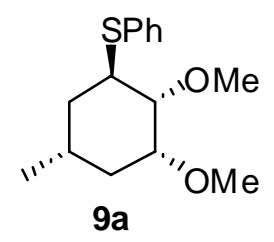

A flame dried flask was charged with $60 \% \mathrm{NaH}$ in oil $(0.58 \mathrm{~g}, 14.5 \mathrm{mmol})$ then with hexanes $(20 \mathrm{~mL})$ and the mixture was cooled to $0^{\circ} \mathrm{C}$ while stirring. Stirring was stopped then, after settling, hexanes was cannulated out and the process was repeated twice. A solution of PhSH (1.54 mL, $15 \mathrm{mmol})$ in THF $(25 \mathrm{~mL})$ was then cannulated slowly to the $\mathrm{NaH}$ suspension and stirring was continued for 30 minutes at $0^{\circ} \mathrm{C}$. This solution was then cannulated slowly to a cold solution of hydroxyepoxide 8a $(1.28 \mathrm{~g}, 10 \mathrm{mmol})$ in THF $(25 \mathrm{~mL})$ and the mixture was allowed to reach ambient temperature while stirring was continued for $3 \mathrm{~h}$. The reaction mixture was cooled again to $0^{\circ} \mathrm{C}$ for $30 \mathrm{~min}$. then $\mathrm{MeI}(7 \mathrm{~mL}, 100 \mathrm{mmol})$ was added and stirring was continued for 30 min. $\mathrm{NaH}(0.44 \mathrm{~g}, 11 \mathrm{mmol})$ was added portionwise under positive $\mathrm{N}_{2}$ 
pressure then the reaction mixture was stirred for additional 30 minutes. MeI (7 ml, $100 \mathrm{mmol}$ ) was added and stirring was continued for $2 \mathrm{~h}$. After completion, ether $(20 \mathrm{~mL})$, brine $(60 \mathrm{~mL})$ were added and the mixture was stirred for 15 minutes. Ice chips were added slowly until a clear two phase mixture was obtained. The organic layer was separated, dried over $\mathrm{Na}_{2} \mathrm{SO}_{4}$ and then was concentrated via rotary evaporation to afford a crude yellow oil. Purification with flash column chromatography $(10 \%$ ethyl acetate/hexanes) afforded the desired sulfide as a clear yellow oil (1.94 g, 81 $\%)$.

Preparation of sulfone $\mathbf{9 b}$ :

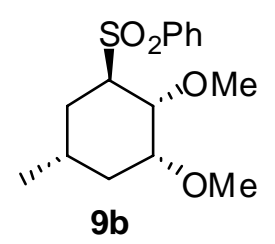

A flame dried flask was charged with sulfide 9a (1.6 g, 6 mmol), dry $\mathrm{CH}_{2} \mathrm{Cl}_{2}$ $(30 \mathrm{~mL})$, and the mixture was cooled to $0^{\circ} \mathrm{C}$ under dry $\mathrm{N}_{2}$ atmosphere for 30 min. $70 \%$ MCPBA $(3.25 \mathrm{~g}, 13.2 \mathrm{mmol})$ was dissolved in dry $\mathrm{CH}_{2} \mathrm{Cl}_{2}(30$ $\mathrm{mL}$ ) and the solution was cannulated slowly into the reaction flask. Stirring was continued for $2 \mathrm{~h}$ and the reaction mixture was allowed to reach ambient temperature. After reaction completion, the reaction mixture was cooled again to $0^{\circ} \mathrm{C}$, washed with aq. saturated $\mathrm{Na}_{2} \mathrm{SO}_{3}$ then with aq. saturated $\mathrm{K}_{2} \mathrm{CO}_{3}$ solution. The organic layer was washed with brine, dried over anhydrous $\mathrm{Na}_{2} \mathrm{SO}_{4}$ then concentrated via rotary evaporation to afford the crude product as a white crystalline solid. Purification by flash column chromatography (20\% ethyl acetate/hexanes) afforded the desired sulfone as a white crystalline solid (1.77 $\mathrm{g}, 99 \%)$. Melting range $81.7-82.7^{\circ} \mathrm{C}$. TLC 
(ethyl acetate/hexanes) $R_{f} 0.17 ;{ }^{1} \mathrm{HNMR}\left(\mathrm{CDCl}_{3}, 300 \mathrm{MHz}\right) \delta 7.80(\mathrm{~m}, 5 \mathrm{H})$, $3.92(\mathrm{t}, J=2.7 \mathrm{~Hz}, 1 \mathrm{H}), 3.82(\mathrm{dt}, J=3.9,11.1 \mathrm{~Hz}, 1 \mathrm{H}), 3.46(\mathrm{dt}, J=3.0,9.0$ $\mathrm{Hz}, 1 \mathrm{H}), 3.40$ (s, 3H), 3.28 (s, 3H), 2.16 ( br. m, 1H), 1.84 (dt, $J=3.6,15.0$ $\mathrm{Hz}, 1 \mathrm{H}), 1.78$ (dt, $J=3.6,12.6 \mathrm{~Hz}, 1 \mathrm{H}), 1.52$ (m, 1H), 1.42 (dt, ap. p, $J=$ 11.1, 23.7 Hz, 1H), $0.94(\mathrm{~d}, J=6.9 \mathrm{~Hz}, 3 \mathrm{H}) ;{ }^{13} \mathrm{CNMR}\left(\mathrm{CDCl}_{3}, 75 \mathrm{MHz}\right) \delta$ $139.1,133.8,129.2,128.1,77.9,74.0,62.5,58.1,56.2,32.3,28.1,26.2$, 22.0; LRMS (CI) calculated for $\mathrm{C}_{15} \mathrm{H}_{22} \mathrm{O}_{4} \mathrm{~S}(\mathrm{M}+)$ 299, found 299; HRMS (EI) calculated for $\mathrm{C}_{15} \mathrm{H}_{22} \mathrm{O}_{4} \mathrm{~S}(\mathrm{M}+)$ 299.1317, found 299.1319.

Alternative procedure for preparation of sulfone $\mathbf{9 b}$ :

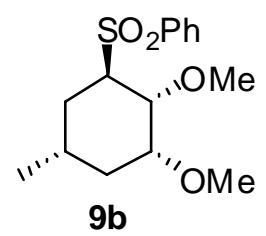

A flame dried flask was charged with sulfide 9a (6 g, $22.5 \mathrm{mmol})$, dry toluene $(120 \mathrm{~mL})$, and the mixture was cooled to $0^{\circ} \mathrm{C}$ under dry $\mathrm{N}_{2}$ atmosphere for 30 minutes. $1 \mathrm{M}$ aq. $\mathrm{Na}_{2} \mathrm{WO}_{4}(0.45 \mathrm{~mL}, 0.45 \mathrm{mmol}), 1 \mathrm{M}$ aq. $\mathrm{PhP}(\mathrm{O})(\mathrm{OH})_{2}(0.45 \mathrm{~mL}, 0.45 \mathrm{mmol})$ and $0.5 \mathrm{M}$ oct $3(\mathrm{Me}) \mathrm{NHSO}_{4}$ in toluene $(0.9 \mathrm{~mL}, 0.45 \mathrm{mmol})$ were added and the mixture was stirred at $0^{\circ} \mathrm{C}$ for 30 minutes. $30 \%$ aq. $\mathrm{H}_{2} \mathrm{O}_{2}(5 \mathrm{~mL}, 45 \mathrm{mmol})$ was cannulated slowly into the reaction flask. The reaction mixture was allowed to reach ambient temperature and stirring was continued for $3 \mathrm{~h}$. After reaction completion, the reaction mixture was cooled again to $0^{\circ} \mathrm{C}$, washed with aq. saturated $\mathrm{Na}_{2} \mathrm{SO}_{3}(100 \mathrm{~mL})$ then with brine $(100 \mathrm{~mL})$. The organic layer was separated, dried over anhydrous $\mathrm{Na}_{2} \mathrm{SO}_{4}$ then concentrated via rotary evaporation to afford the crude product as a white crystalline solid. 
Purification by flash column chromatography (20\% ethyl acetate/hexanes) afforded the desired sulfone as a white crystalline solid $(6.66 \mathrm{~g}, 99 \%){ }^{2}$

Preparation of vinyl sulfone 10:

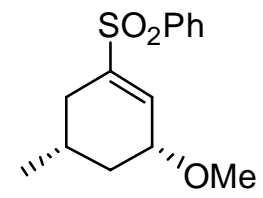

10

A solution of sulfone $9 \mathbf{b}(1.49 \mathrm{~g}, 6 \mathrm{mmol})$ in THF $(60 \mathrm{~mL})$ was cooled to $0^{\circ} \mathrm{C}$ for 15 minutes then $\mathrm{NaH}(0.28 \mathrm{~g}, 7 \mathrm{mmol})$ was added portionwise under positive dry $\mathrm{N}_{2}$ atmosphere. The mixture was allowed to reach ambient temperature while stirring was continued for $3 \mathrm{~h}$. After completion, the reaction mixture was diluted with ether $(30 \mathrm{~mL})$, cooled to $0^{\circ} \mathrm{C}$, and ice chips were added slowly until a clear solution was obtained. The organic layer was washed with brine $(90 \mathrm{~mL})$, dried over $\mathrm{Na}_{2} \mathrm{SO}_{4}$, and then was concentrated via rotary evaporation to afford a crude yellow oil. Purification with flash column chromatography (10\% ethyl acetate/hexanes) afforded 10 as a colorless oil $(1.36 \mathrm{~g}, 85 \%)$ that solidified overnight at $-20^{\circ} \mathrm{C}$. Melting range is $67.5-68.3^{\circ} \mathrm{C}$. TLC ( $30 \%$ ethyl acetate/hexanes) $R_{f} \quad 0.40 ;{ }^{1} \mathrm{H}$ NMR $\left(\mathrm{CDCl}_{3}, 300 \mathrm{MHz}\right) \delta 7.80(\mathrm{~m}, 2 \mathrm{H}), 7.56(\mathrm{~m}, 3 \mathrm{H}), 6.96(\mathrm{~s}, 1 \mathrm{H}), 3.98(\mathrm{~m}$, 1H), $3.40(\mathrm{~s}, 3 \mathrm{H}), 2.32(\mathrm{dd}, J=2.1,4.5,16.8 \mathrm{~Hz}, 1 \mathrm{H}), 2.02(\mathrm{~m}, 1 \mathrm{H}), 1.78$ (br. m, 1H), 1.60 (m, 1H), 1.42 (ap. s, $1 \mathrm{H}), 1.06$ (dt, $J=10.2,12.3 \mathrm{~Hz}, 1 \mathrm{H})$; $0.98(\mathrm{~d}, J=6.6 \mathrm{~Hz}, 3 \mathrm{H}) ;{ }^{13} \mathrm{C} \mathrm{NMR}\left(\mathrm{CDCl}_{3}, 75 \mathrm{MHz}\right) \delta 141.8,139.0,137.0$, 133.8, 129.0, 128.1, 76.2, 56.2, 36.1, 31.7, 28.1, 21.8; LRMS (EI) calculated

\footnotetext{
${ }^{2}$ Usually no chromatography is needed when Noyori's oxidation is employed. However, chromatography was used here to get rid of the phase transfer catalyst $\left(\operatorname{oct}_{3}(\mathrm{Me}) \mathrm{NHSO}_{4}\right)$ and confirm the yield.
} 
for $\mathrm{C}_{14} \mathrm{H}_{18} \mathrm{O}_{3} \mathrm{~S}(\mathrm{M}+)$ 266, found 266; HRMS (M+) calculated for $\mathrm{C}_{14} \mathrm{H}_{18} \mathrm{O}_{3} \mathrm{~S}$ 266.0977, found 266.0977.

This procedure was scaled up using sulfone 9b (10 g, $33.4 \mathrm{mmol})$ and yielded vinyl sulfone 10 as a white crystalline solid (7.5 g, 85\%).

Preparation of aldehyde ester 11:

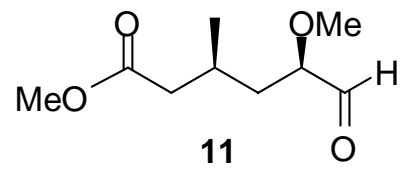

A solution of vinyl sulfone $10(0.53 \mathrm{~g}, 2 \mathrm{mmol})$ in anhydrous $\mathrm{MeOH}$ was treated with trimethyl orthoformate $(2.2 \mathrm{~mL}, 20 \mathrm{mmol})$ and the mixture was stirred at room temperature for 30 minutes. Sodium bicarbonate $(0.84 \mathrm{~g}, 10$ mmol) was added then the mixture was cooled to $-78^{\circ} \mathrm{C}$ under positive $\mathrm{O}_{2}$ pressure. The mixture was slowly ozonized at $-78^{\circ} \mathrm{C}$ for 15 minutes then the ozonation was stopped and the reaction was purged with dry $\mathrm{N}_{2}$ for 30 minutes. Dimethylsulfide $(0.3 \mathrm{~mL}, 4 \mathrm{mmol})$ was added and the mixture was allowed to reach ambient temperature while stirring took place under dry $\mathrm{N}_{2}$ atmosphere for $4 \mathrm{~h}$. The mixture was concentrated via rotary evaporation to afford 11 as a colorless oil (0.346 g, 92\%). ${ }^{3}$ TLC (10\% ethyl acetate/hexanes) $R_{f} 0.24 ;{ }^{1} \mathrm{H} \mathrm{NMR}\left(\mathrm{CDCl}_{3}, 300 \mathrm{MHz}\right), \delta 9.60(\mathrm{~d}, J=1.8 \mathrm{~Hz}$, 1H), 3.62 (s, 3H), 3.60 (ddd, $J=2.1,5.1,7.8 \mathrm{~Hz}, 1 \mathrm{H}), 3.42$ (s, 3H), 2.38 (m, $1 \mathrm{H}), 2.16(\mathrm{~m}, 2 \mathrm{H}), 1.58(\mathrm{~m}, 2 \mathrm{H}), 0.96(\mathrm{~d}, J=6.4 \mathrm{~Hz}, 3 \mathrm{H}) ;{ }^{13} \mathrm{C} \mathrm{NMR}\left(\mathrm{CDCl}_{3}\right.$, $75 \mathrm{MHz}) \delta 203.2,172.8,83.9,58.1,51.7,41.2,36.1,26.9,20.4$; LRMS (CI) calculated for $\mathrm{C}_{9} \mathrm{H}_{16} \mathrm{O}_{4}(\mathrm{M}+)$ 188.22, found 189 .

\footnotetext{
${ }^{3}$ Attempts to purify aldehyde $\mathbf{1 1}$ by column chromatography resulted in decomposition/isomerization.
} 
Preparation of vinyl phosphonate 21:

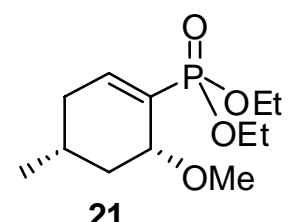

Following general procedure (A) or (B) and starting from vinyl sulfone 10 (266 mg, $1 \mathrm{mmol}$ ), 21 was obtained as a colorless oil in 93\% and 87\% respectively. TLC (80\% ethyl acetate/hexanes) $R_{f} 0.31 ;{ }^{1} \mathrm{H} \mathrm{NMR}\left(\mathrm{CDCl}_{3}\right.$, $300 \mathrm{MHz}) \delta 6.78(\mathrm{dd}, J=5.8,22.2 \mathrm{~Hz}, 1 \mathrm{H}), 4.10$ (m, 4H), $3.42(\mathrm{~s}, 3 \mathrm{H}), 2.20$ (br. m, 2H), 2.04 (ap. br. s, 1H), 1.78 (br. m, 1H), 1.38 (t, $J=6.8 \mathrm{~Hz}, 6 \mathrm{H}$ ), $1.22(\mathrm{q}, J=11.0 \mathrm{~Hz}, 1 \mathrm{H}), 1.01(\mathrm{~d}, J=6.1 \mathrm{~Hz}, 3 \mathrm{H}) ;{ }^{13} \mathrm{C} \mathrm{NMR}\left(\mathrm{CDCl}_{3}, 75\right.$ MHz) $\delta 140.5(\mathrm{~d}, J=5.2 \mathrm{~Hz}), 76.1(\mathrm{~d}, J=5.8 \mathrm{~Hz}), 61.8(\mathrm{dd}, J=5.3,9.2$ $\mathrm{Hz}), 56.8,36.4(\mathrm{~d}, J=10.5 \mathrm{~Hz}), 34.6(\mathrm{~d}, J=18.5 \mathrm{~Hz}), 27.4,21.7,16.4(\mathrm{t}, J=$ $3.2 \mathrm{~Hz}) ;{ }^{31} \mathrm{P} \mathrm{NMR}\left(\mathrm{CDCl}_{3}, 282.216 \mathrm{MHz}\right) \delta 19.5$; LRMS (EI) calculated for $\mathrm{C}_{12} \mathrm{H}_{23} \mathrm{O}_{4} \mathrm{P}(\mathrm{M}+\mathrm{H})$ 263, found 263; HRMS (EI) calculated for $\mathrm{C}_{12} \mathrm{H}_{23} \mathrm{O}_{4} \mathrm{P}$ $(\mathrm{M}+\mathrm{H})$ 263.1412, found 263.1421. 
Preparation of aldehyde ester 14:

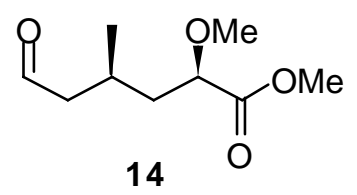

A solution of vinyl phosphonate $21(1.05 \mathrm{~g}, 4 \mathrm{mmol})$ in dry $\mathrm{CH}_{2} \mathrm{Cl}_{2} / \mathrm{MeOH}$ (4:1) was treated with trimethyl orthoformate $(4.38 \mathrm{~mL}, 40 \mathrm{mmol})$ and the mixture was stirred at room temperature for 30 minutes. Sodium bicarbonate $(1.68 \mathrm{~g}, 20 \mathrm{mmol})$ was added then the mixture was cooled to $-78^{\circ} \mathrm{C}$ under positive $\mathrm{O}_{2}$ pressure. The mixture was slowly ozonized at $-78^{\circ} \mathrm{C}$ for 30 minutes then the ozonation was stopped and the reaction was purged with dry $\mathrm{N}_{2}$ for 30 minutes. Triphenylphosphine (1.32 g, $\left.5 \mathrm{mmol}\right)$ was added and the mixture was allowed to reach ambient temperature while stirring took place under dry $\mathrm{N}_{2}$ atmosphere for $2 \mathrm{~h}$. The mixture was concentrated via rotary evaporation ${ }^{4}$ then was purified by flash column chromatography (10\% ethyl acetate/hexanes) to afford 14 as a colorless oil $(0.67 \mathrm{~g}, 89 \%)$. TLC (30\% ethyl acetate/hexanes) $R_{f} \quad 0.40 ;{ }^{1} \mathrm{H}$ NMR $\left(\mathrm{CDCl}_{3}, 300 \mathrm{MHz}\right), \delta$ $9.62(\mathrm{~d}, J=1.8 \mathrm{~Hz}, 1 \mathrm{H}), 3.68(\mathrm{~s}, 3 \mathrm{H}), 3.62(\mathrm{dt}, J=1.8,5.3 \mathrm{~Hz}, 1 \mathrm{H}), 3.42(\mathrm{~s}$, $3 \mathrm{H}), 2.39(\mathrm{~m}, 1 \mathrm{H}), 2.22(\mathrm{~m}, 2 \mathrm{H}), 1.62(\mathrm{~m}, 2 \mathrm{H}), 1.01(\mathrm{~d}, J=6.5 \mathrm{~Hz}, 3 \mathrm{H}) ;{ }^{13} \mathrm{C}$ NMR $\left(\mathrm{CDCl}_{3}, 75 \mathrm{MHz}\right) \delta 203.8,172.9,84.1,58.1,51.8,41.0,36.1,26.6$, 20.8; LRMS (CI) calculated for $\mathrm{C}_{9} \mathrm{H}_{16} \mathrm{O}_{4}(\mathrm{M}+\mathrm{H})$ 189, found 189; HRMS (EI) calculated for $\mathrm{C}_{9} \mathrm{H}_{16} \mathrm{O}_{4}(\mathrm{M}+\mathrm{H})$ 189.1127, found 189.1127 .

\footnotetext{
${ }^{4}$ Breaking the vacuum must be performed under argon since aldehyde $\mathbf{1 4}$ is extremely sensitive to $\mathrm{O}_{2}$ and oxidizes immediately to the carboxylic acid.
} 
Preparation of phosphonate sulfone adduct 22:

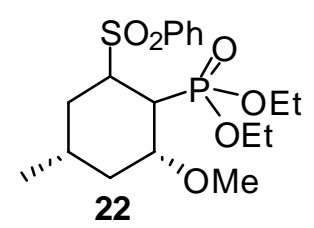

Following general procedure (A) or (D) and starting from vinyl sulfone 10 (266 mg, $1 \mathrm{mmol}$ ), adduct 22 was obtained in 7\% and $97 \%$ yield respectively. TLC (80\% ethyl acetate/hexanes) $R_{f} 0.44 ;{ }^{1} \mathrm{H}$ NMR $\left(\mathrm{CDCl}_{3}\right.$, $300 \mathrm{MHz}) \delta 7.83(\mathrm{dt}, J=1.5,6.9 \mathrm{~Hz}, 2 \mathrm{H}), 7.63(\mathrm{tt}, J=1.2,7.5 \mathrm{~Hz}, 1 \mathrm{H}), 7.58$ (t, $J=7.8 \mathrm{~Hz}, 2 \mathrm{H}), 4.00(\mathrm{~m}, 5 \mathrm{H}), 3.80(\mathrm{dd}, J=5.7,14.7 \mathrm{~Hz}, 1 \mathrm{H}), 3.40(\mathrm{~s}$, $3 \mathrm{H}$ ), 3.10 (dd, $J=6.0,23.7 \mathrm{~Hz}, 1 \mathrm{H}$ ), 2.18 (br. m, 1H), 2.00 (d, $J=15.0 \mathrm{~Hz}$, $1 \mathrm{H}), 1.85$ (dt, $J=4.2,12.3 \mathrm{~Hz}, 1 \mathrm{H}), 1.70$ (q, $J=12.3 \mathrm{~Hz}, 1 \mathrm{H}), 1.60(\mathrm{~m}, 1 \mathrm{H})$, $1.22(\mathrm{dt}, J=6.9,12.9 \mathrm{~Hz}, 6 \mathrm{H}), 0.93(\mathrm{~d}, J=6.6 \mathrm{~Hz}, 3 \mathrm{H}) ;{ }^{13} \mathrm{C} \mathrm{NMR}\left(\mathrm{CDCl}_{3}\right.$, $75 \mathrm{MHz}) \delta 137.8,133.8,129.4,128.4,75.8(\mathrm{~d}, J=6.6 \mathrm{~Hz}), 62.5(\mathrm{~d}, J=6.1$ Hz), 61.4, 61.2 (d, $J=6.9 \mathrm{~Hz}), 56.7,35.6(\mathrm{~d}, J=16.0 \mathrm{~Hz}), 33.7,29.4,26.3$, 22.1, $16.4(\mathrm{dd}, J=5.8,16.5 \mathrm{~Hz}) ;{ }^{31} \mathrm{P}$ NMR $\left(\mathrm{CDCl}_{3}, 282.216 \mathrm{MHz}\right) \delta 26.0$; LRMS (CI) calculated for $\mathrm{C}_{18} \mathrm{H}_{29} \mathrm{SO}_{6} \mathrm{P}(\mathrm{M}+\mathrm{H})$ 405, found, 405; HRMS (EI) calculated for $\mathrm{C}_{18} \mathrm{H}_{29} \mathrm{SO}_{6} \mathrm{P}(\mathrm{M}+\mathrm{H})$ 405.1501, found 405.1495. 
Preparation of vinyl phosphonate 24a:

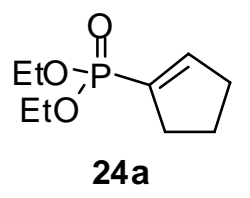

Following general procedure (A) and starting from vinyl sulfone 24 (208 mg, $1 \mathrm{mmol}$ ), vinyl phosphonate $\mathbf{2 4 a}$ was received as a crude colorless oil. Purification by flash column chromatography (50\% ethylacetate/hexanes) afforded 24a as a colorless oil (193 mg, 95\%). TLC (40\% ethyl acetate/hexanes) $R_{f} 0.15 ;{ }^{1} \mathrm{H} \mathrm{NMR}\left(\mathrm{CDCl}_{3}, 300 \mathrm{MHz}\right) \delta 6.58(\mathrm{dp}, J=2.1$, $11.4 \mathrm{~Hz}, 1 \mathrm{H}), 4.00(\mathrm{dq}, J=7.5,14.1 \mathrm{~Hz}, 4 \mathrm{H}), 2.45(\mathrm{~m}, 4 \mathrm{H}), 1.90(\mathrm{~m}, 2 \mathrm{H})$, $1.26(\mathrm{t}, J=7.2 \mathrm{~Hz}, 6 \mathrm{H}) ;{ }^{13} \mathrm{C} \mathrm{NMR}\left(\mathrm{CDCl}_{3}, 75 \mathrm{MHz}\right) \delta 146.8(\mathrm{~J}=14.3 \mathrm{~Hz})$, $132.4(\mathrm{~d}, J=188.2 \mathrm{~Hz}), 61.5(\mathrm{~d}, J=5.4 \mathrm{~Hz}), 34.1$ (d, $J=21.4 \mathrm{~Hz}), 33.0$ (d, $J$ $=13.8 \mathrm{~Hz}), 23.6(\mathrm{~d}, J=12.3 \mathrm{~Hz}), 16.3(\mathrm{~d}, J=6.2 \mathrm{~Hz}) ;{ }^{31} \mathrm{P} \mathrm{NMR}\left(\mathrm{CDCl}_{3}\right.$, 282.216 MHz) $\delta$ 17.5; LRMS (EI) calculated for $\mathrm{C}_{9} \mathrm{H}_{17} \mathrm{O}_{3} \mathrm{P}(\mathrm{M}+\mathrm{H})$ 205, found 205; HRMS (EI) calculated for $\mathrm{C}_{9} \mathrm{H}_{17} \mathrm{O}_{3} \mathrm{P}(\mathrm{M}+$ ) 204.0915, found 204.0916 . 
Preparation of vinyl phosphonate 25a:

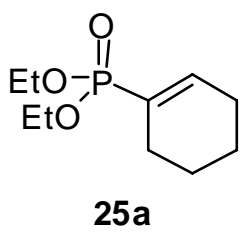

Following general procedure (A) and starting from vinyl sulfone 25 (222 mg, $1 \mathrm{mmol}$ ), vinyl phosphonate 25a was received as a crude colorless oil. Purification by flash column chromatography (40\% ethylacetate/hexanes) afforded 25a as a colorless oil (196 mg, 90\%). TLC (40\% ethyl acetate/hexanes) $R_{f} 0.22 ;{ }^{1} \mathrm{H} \mathrm{NMR}\left(\mathrm{CDCl}_{3}, 300 \mathrm{MHz}\right) \delta 6.75(\mathrm{dp}, J=1.8$, $22.5 \mathrm{~Hz}, 1 \mathrm{H}), 4.00(\mathrm{~m}, 4 \mathrm{H}), 2.18(\mathrm{~m}, 4 \mathrm{H}), 1.60(\mathrm{~m}, 4 \mathrm{H}), 1.26(\mathrm{t}, J=6.9 \mathrm{~Hz}$, $6 \mathrm{H}) ;{ }^{13} \mathrm{C} \mathrm{NMR}\left(\mathrm{CDCl}_{3}, 75 \mathrm{MHz}\right) \delta 143.3(\mathrm{~d}, J=9.1 \mathrm{~Hz}), 127.4(\mathrm{~d}, J=179.7$ Hz), $61.4(\mathrm{~d}, J=5.2 \mathrm{~Hz}), 25.9$ (d, $J=18.1 \mathrm{~Hz}), 24.2(\mathrm{~d}, J=8.9 \mathrm{~Hz}), 21.8$ (d, $J=10.1 \mathrm{~Hz}), 21.3,16.3(\mathrm{~d}, J=6.2 \mathrm{~Hz}) ;{ }^{31} \mathrm{P} \mathrm{NMR}\left(\mathrm{CDCl}_{3}, 282.216 \mathrm{MHz}\right) \delta$ 20.7; LRMS (EI) calculated for $\mathrm{C}_{10} \mathrm{H}_{19} \mathrm{O}_{3} \mathrm{P}(\mathrm{M}+)$ 218, found 218; HRMS (EI) calculated for $\mathrm{C}_{10} \mathrm{H}_{19} \mathrm{O}_{3} \mathrm{P}(\mathrm{M}+)$ 218.1072, found 218.1072. 
Preparation of vinyl phosphonate 18a:

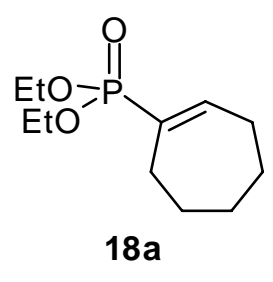

Following general procedure (A) and starting from vinyl sulfone 18 (236 mg, $1 \mathrm{mmol}$ ), vinyl phosphonate 18a was received as a crude colorless oil. Purification by flash column chromatography ( $40 \%$ ethylacetate/hexanes) afforded 18a as a colorless oil (218 mg, 94\%). TLC (40\% ethyl acetate/hexanes) $R_{f} 0.20 ;{ }^{1} \mathrm{H}$ NMR $\left(\mathrm{CDCl}_{3}, 300 \mathrm{MHz}\right) \delta 6.90(\mathrm{dt}, J=6.3$, $23.7 \mathrm{~Hz}, 1 \mathrm{H}), 4.00(\mathrm{~m}, 4 \mathrm{H}), 2.25(\mathrm{~m}, 4 \mathrm{H}), 1.76(\mathrm{~m}, 2 \mathrm{H}), 1.45(\mathrm{~m}, 4 \mathrm{H}), 1.24$ $(\mathrm{t}, J=7.7 \mathrm{~Hz}, 6 \mathrm{H}) ;{ }^{13} \mathrm{C} \mathrm{NMR}\left(\mathrm{CDCl}_{3}, 75 \mathrm{MHz}\right) \delta 148.9(\mathrm{~d}, J=9.4 \mathrm{~Hz})$, $133.0(\mathrm{~d}, J=187.5 \mathrm{~Hz}), 61.5(\mathrm{~d}, J=3.4 \mathrm{~Hz}), 32.4,28.7(\mathrm{~d}, J=20.5 \mathrm{~Hz})$, $28.3(\mathrm{~d}, J=13.6 \mathrm{~Hz}), 26.7(\mathrm{~d}, J=6.8 \mathrm{~Hz}), 25.8,16.3(\mathrm{~d}, J=6.8 \mathrm{~Hz}) ;{ }^{31} \mathrm{P}$ NMR $\left(\mathrm{CDCl}_{3}, 282.216 \mathrm{MHz}\right) \delta 22.6$; LRMS (EI) calculated for $\mathrm{C}_{11} \mathrm{H}_{21} \mathrm{O}_{3} \mathrm{P}$ $(\mathrm{M}+)$ 232, found 232; HRMS (EI) calculated for $\mathrm{C}_{11} \mathrm{H}_{21} \mathrm{O}_{3} \mathrm{P}(\mathrm{M}+)$ 232.1228, found 232.1224 . 
Characterization of dienyl phosphonate 27b:

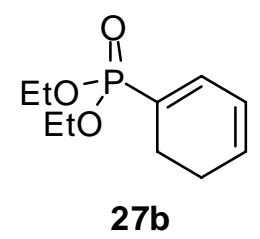

Following procedure (A) and starting from vinyl sulfone 27 (238 mg, 1 mmol), dienyl sulfone 27b was obtained as a crude colorless oil. Purification by flash column chromatography ( $80 \%$ ethyl acetate/hexane) afforded $27 \mathbf{b}$ as a colorless oil (181 mg, 84\%). TLC (80\% ethyl acetate/hexanes) $R_{f} 0.20$. ${ }^{1} \mathrm{H}$ NMR $\left(\mathrm{CDCl}_{3}, 300 \mathrm{MHz}\right) \delta 6.79$ (ddd, $\left.J=2.0,4.7,20.2 \mathrm{~Hz}\right), 6.08$ (m, 2H), $4.08(\mathrm{~m}, 4 \mathrm{H}), 2.28(\mathrm{~m}, 4 \mathrm{H}), 1.34(\mathrm{t}, J=7.3 \mathrm{~Hz}, 6 \mathrm{H}) ;{ }^{13} \mathrm{C} \mathrm{NMR}\left(\mathrm{CDCl}_{3}\right.$, $75 \mathrm{MHz}) \delta 136.8(\mathrm{~d}, J=9.8 \mathrm{~Hz}), 132.4(\mathrm{~d}, J=4.0 \mathrm{~Hz}), 124.1(\mathrm{~d}, J=189.4$ Hz), $123.7(\mathrm{~d}, J=18.7 \mathrm{~Hz}), 61.7(\mathrm{~d}, J=4.5 \mathrm{~Hz}), 22.2(\mathrm{~d}, J=9.4 \mathrm{~Hz}), 21.2$ $(\mathrm{d}, J=9.9 \mathrm{~Hz}), 16.4(\mathrm{~d}, J=6.2 \mathrm{~Hz}) ;{ }^{31} \mathrm{P}$ NMR $\left(\mathrm{CDCl}_{3}, 282.216 \mathrm{MHz}\right) \delta$ 20.1; LRMS (CI) calculated for $\mathrm{C}_{10} \mathrm{H}_{17} \mathrm{O}_{3} \mathrm{P}(\mathrm{M}+)$ 216, found $217(\mathrm{M}+\mathrm{H})$. 
Preparation of Vinyl sulfone 28:

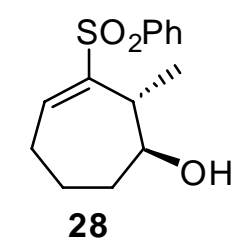

A solution of epoxide $31(5 \mathrm{~g}, 20 \mathrm{mmol})$ in dry $\mathrm{CH}_{2} \mathrm{Cl}_{2}(50 \mathrm{~mL})$ was cooled to $-78^{\circ} \mathrm{C}$ for 30 minutes then $25 \% \mathrm{AlMe}_{3}$ in hexanes $(30 \mathrm{~mL}, 60 \mathrm{mmol})$ was added dropwise. The dry ice bath was removed and the reaction was left stirring for $24 \mathrm{~h}$. Reaction was checked for completion using TLC (50\% ethyl acetate/hexanes) then the reaction mixture was cooled again to $-78^{\circ} \mathrm{C}$ for 30 minutes. Careful and dropwise addition of aq. $10 \% \mathrm{NaOH}$ (until $\mathrm{CH}_{4}$ gas evolution stopped) was followed by adding aq. 10\% $\mathrm{NaOH}(50 \mathrm{~mL})$ then the mixture was allowed to stir at ambient temperature for 30 minutes. Organic phase was separated, washed with brine $(50 \mathrm{~mL})$, dried over $\mathrm{Na}_{2} \mathrm{SO}_{4}$ then was concentrated via rotary evaporation to afford a crude yellowish oil (de 10:1). Purification by flash column chromatography (20\% ethyl acetate/hexanes) afforded 28 as a colorless oil (4.5 g, 85\%). Crystallization from ether gave $\mathbf{2 8}$ as white prismic crystals (3.2 g, 60\%). Melting range $74.8-75.4^{\circ} \mathrm{C}$. TLC (50\% ethyl acetate/hexanes) $R_{f} 0.26 .{ }^{1} \mathrm{H}$ NMR $\left(\mathrm{CDCl}_{3}, 300 \mathrm{MHz}\right) \delta 7.88(\mathrm{~d}, J=8.2 \mathrm{~Hz}, 2 \mathrm{H}), 7.58(\mathrm{~m}, 3 \mathrm{H}), 7.37$ (ddd, $J=1.1,4.1,9.4 \mathrm{~Hz}, 1 \mathrm{H}$ ), 3.82 (br. m, 1H), 2.91 (ap. p, $J=7.2 \mathrm{~Hz}, 1 \mathrm{H}$ ), 2.48 (m, 1H), 2.27 (tt, $J=4.5,13.0 \mathrm{~Hz}, 1 \mathrm{H}), 1.98$ (m, 1H), 1.87 (dt, $J=3.1$, $12.6 \mathrm{~Hz}, 1 \mathrm{H}), 1.78(\mathrm{~m}, 1 \mathrm{H}), 1.68(\mathrm{dt}, J=2.0,12.2 \mathrm{~Hz}, 1 \mathrm{H}), 1.54(\mathrm{~m}, 1 \mathrm{H})$, $1.44(\mathrm{~d}, J=2.7 \mathrm{~Hz}, 1 \mathrm{H}), 0.92(\mathrm{~d}, J=7.0 \mathrm{~Hz}, 3 \mathrm{H}) ;{ }^{13} \mathrm{C}$ NMR $\left(\mathrm{CDCl}_{3}, 75\right.$ MHz) $\delta 144.1,142.4,138.8,133.2,129.2,128.4,69.4,39.9,31.4,27.3$, 
18.2, 15.6; LRMS (EI) calculated for $\mathrm{C}_{14} \mathrm{H}_{18} \mathrm{O}_{3} \mathrm{~S}(\mathrm{M}+)$ 266, found 266; HRMS (EI) calculated for $\mathrm{C}_{14} \mathrm{H}_{18} \mathrm{O}_{3} \mathrm{~S}(\mathrm{M}+)$ 266.0977, found 266.0978.

Preparation of caged phosphonate 28a:

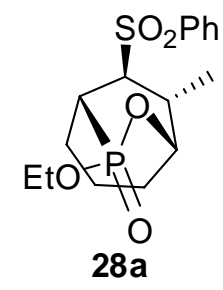

Following general procedure (B) and starting from vinyl sulfone 28 (266 $\mathrm{mg}, 1 \mathrm{mmol}$ ), 28a was received as a crude yellow oil. Purification by flash column chromatography (80\% ethyl acetate/hexanes) afforded 28a as white needle crystals $(0.311 \mathrm{~g}, 87 \%)$. Melting range $166.5-168^{\circ} \mathrm{C}$. TLC (80\% ethyl acetate/hexanes) $R_{f} 0.17 ;{ }^{1} \mathrm{H}$ NMR $\left(\mathrm{CDCl}_{3}, 300 \mathrm{MHz}\right) \delta 7.89(\mathrm{~d}, J=7.3 \mathrm{~Hz}$, 2H), 7.69 (tt, $J=0.9,6.3 \mathrm{~Hz}, 1 \mathrm{H}), 7.60$ (tt, $J=1.4,8.3 \mathrm{~Hz}, 2 \mathrm{H}), 4.40$ (ddt, $J$ $=2.1,4.2,22.4 \mathrm{~Hz}, 1 \mathrm{H}), 4.15(\mathrm{~m}, 2 \mathrm{H}), 3.26(\mathrm{dd}, J=11.0,24.9 \mathrm{~Hz}, 1 \mathrm{H}), 2.98$ (br. m, 1H), 2.86 (dt, $J=4.6,20.3 \mathrm{~Hz}, 1 \mathrm{H}), 2.35$ (m, 1H), 2.08 (m, 2H), 1.76 (m, 2H), 1.62 (m, 1H), 1.48 (br. m, 1H), 1.36 (t, $J=7.3 \mathrm{~Hz}, 3 \mathrm{H}), 1.05$ (d, $J=$ $6.5 \mathrm{~Hz}, 3 \mathrm{H}) ;{ }^{13} \mathrm{C} \mathrm{NMR}\left(\mathrm{CDCl}_{3}, 75 \mathrm{MHz}\right) \delta 139.8,133.9,129.4,128.2,90.1$ $(\mathrm{d}, J=5.6 \mathrm{~Hz}), 70.6(\mathrm{~d}, J=4.4 \mathrm{~Hz}), 63.0(\mathrm{~d}, J=7.2 \mathrm{~Hz}), 34.2(\mathrm{~d}, J=15.7$ $\mathrm{Hz}), 30.6,29.6,28.8,26.4,19.4,17.4,16.3(\mathrm{~d}, J=6.3 \mathrm{~Hz}) ;{ }^{31} \mathrm{P}$ NMR $\left(\mathrm{CDCl}_{3}, 282.216 \mathrm{MHz}\right) \delta 24.1$; LRMS (EI) calculated for $\mathrm{C}_{16} \mathrm{H}_{23} \mathrm{O}_{5} \mathrm{PS}(\mathrm{M}+)$ 358, found 358; HRMS (EI) calculated for $\mathrm{C}_{16} \mathrm{H}_{23} \mathrm{O}_{5} \mathrm{PS}(\mathrm{M}+)$ 358.1004, found 358.1018 . 
Characterization of $\mathbf{2 8 b}$ :

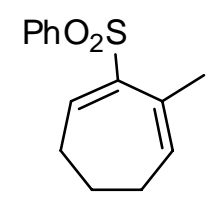

$28 b$

A solution of diisopropylamine $(0.15 \mathrm{ml}, 1.05 \mathrm{mmol})$ in THF $(3 \mathrm{~mL})$ was cooled to $-78^{\circ} \mathrm{C}$ for 10 minutes then was treated with $\mathrm{n}$-BuLi $(0.4 \mathrm{~mL}, 1$ mmol) dropwisely. The mixture was allowed to warm up to ambient temperature and was stirred for 30 minutes then was cooled to $-78^{\circ} \mathrm{C}$ for 10 minutes (solution A). To a cold solution stirred at $-78^{\circ} \mathrm{C}$ of vinylsulfone $\mathbf{2 8}$ (266 mg, $1 \mathrm{mmol})$ in THF $(2 \mathrm{~mL})$ was added solution A dropwise via cannula and the mixture was allowed to reach ambient temperature for 30 minutes then was cooled again to $-78^{\circ} \mathrm{C}$ for 15 minutes (solution $\mathrm{B}$ ). In a separate flask, diethylphosphite $(0.146 \mathrm{~mL}, 1.1 \mathrm{mmol})$ in THF $(3 \mathrm{~mL})$ was treated with NaHMDS $(0.5 \mathrm{~mL}, 1 \mathrm{mmol})$ dropwise at ambient temperature and the mixture was stirred for 15 minutes then was cooled to $-78^{\circ} \mathrm{C}$ for 15 minutes (solution C). Solution $\mathrm{C}$ was cannulated dropwise to solution B at $78^{\circ} \mathrm{C}$ and the mixture was allowed to stir at $-78^{\circ} \mathrm{C}$ for 30 minutes. The dryice bath was removed and the mixture was allowed to warm up to ambient temperature while stirring for $3 \mathrm{~h}$. The reaction was diluted with ether (4 $\mathrm{mL})$ then saturated aq. $\mathrm{NH}_{4} \mathrm{Cl}$ was added at $0^{\circ} \mathrm{C}$ followed by brine $(10 \mathrm{~mL})$. The organic layer was separated, dried over $\mathrm{Na}_{2} \mathrm{SO}_{4}$ and then concentrated via rotary evaporation to give a crude yellowish oil. Purification by flash column chromatography (10\% ethyl acetate/hexane) afforded $\mathbf{2 8 b}$ as a white crystalline solid. Melting range $74.2-76.3^{\circ} \mathrm{C}$. TLC $(10 \%$ ethyl acetate/hexanes) $R_{f}$ 0.32. ${ }^{1} \mathrm{H} \mathrm{NMR}\left(\mathrm{CDCl}_{3}, 300 \mathrm{MHz}\right) \delta 7.87(\mathrm{~m}, 2 \mathrm{H}), 7.55$ 
(m, 3H), $6.02(\mathrm{dt}, J=1.3,10.4 \mathrm{~Hz}, 1 \mathrm{H}), 5.53(\mathrm{ddd}, J=2.4,6.3,10.2 \mathrm{~Hz}$, 1H), $2.32(\mathrm{~m}, 1 \mathrm{H}), 1.95(\mathrm{~m}, 2 \mathrm{H}), 1.72(\mathrm{~m}, 1 \mathrm{H}), 1.58(\mathrm{~m}, 1 \mathrm{H}), 1.57(\mathrm{~s}, 3 \mathrm{H})$, $1.50(\mathrm{~m}, 1 \mathrm{H}) ;{ }^{13} \mathrm{C} \mathrm{NMR}\left(\mathrm{CDCl}_{3}, 75 \mathrm{MHz}\right) \delta 140.7,133.1,128.9,127.8$, 126.5, 123.9, 44.1, 30.6, 29.2, 20.1, 16.9, 12.9; LRMS (CI) calculated for $\mathrm{C}_{14} \mathrm{H}_{16} \mathrm{O}_{2} \mathrm{~S}(\mathrm{M}+)$ 248, found $249(\mathrm{M}+\mathrm{H})$; HRMS (EI) calculated for $\mathrm{C}_{14} \mathrm{H}_{16} \mathrm{O}_{2} \mathrm{~S}(\mathrm{M}+)$ 248.0871, found 248.0870 (M+).

Preparation of 1,4-adduct 31a:

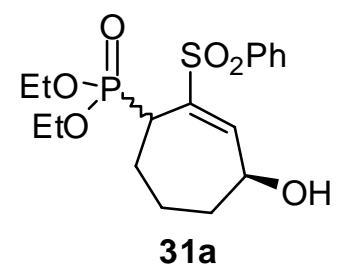

To a solution of epoxide $31(0.25 \mathrm{~g}, 1 \mathrm{mmol})$, diethyl phosphate $(0.146 \mathrm{~mL}$, $1.1 \mathrm{mmol})$ in THF $(2 \mathrm{~mL})$ was added KHMDS (100 uL, $0.088 \mathrm{mmol})$ dropwise at ambient temperature and the mixture was stirred for 30 minutes. Reaction completion was checked by TLC ( $60 \%$ ethyl acetate/hexanes) then brine $(2 \mathrm{~mL})$ was added and the mixture was stirred for additional 15 minutes. Organic phase was separated, dried over $\mathrm{Na}_{2} \mathrm{SO}_{4}$ and was concentrated via rotary evaporation to afford a crude yellow oil. Purification by flash column chromatography (60\% ethyl acetate/hexanes) afforded 31a as a colorless oil $(0.3 \mathrm{~g}, 85 \%)$. TLC (60\% ethyl acetate/hexanes) $R_{f} 0.27{ }^{1} \mathrm{H}$ NMR $\left(\mathrm{CDCl}_{3}, 300 \mathrm{MHz}\right) \delta 7.76(\mathrm{~d}, J=0.9,6.0,2 \mathrm{H}), 7.50(\mathrm{~m}, 4 \mathrm{H}), 6.18$ (br. d, $J=10.7 \mathrm{~Hz}, 1 \mathrm{H}$; exchange $\left.\mathrm{D}_{2} \mathrm{O}\right), 4.36$ (br. s, $\left.1 \mathrm{H}\right), 4.06(\mathrm{~m}, 2 \mathrm{H}), 4.02(\mathrm{~m}$, 1H), $3.84(\mathrm{~m}, 1 \mathrm{H}), 3.28(\mathrm{dt}, J=4.2,31.5 \mathrm{~Hz}, 1 \mathrm{H}), 2.34(\mathrm{q}, J=13.7 \mathrm{~Hz}, 1 \mathrm{H})$, $2.02(\mathrm{~m}, 2 \mathrm{H}), 1.60$ (br. d, $J=15.0 \mathrm{~Hz}, 1 \mathrm{H}), 1.36$ (tt, $J=2.5,15.0 \mathrm{~Hz}, 1 \mathrm{H})$, 
$1.25(\mathrm{t}, J=7.0 \mathrm{~Hz}, 3 \mathrm{H}), 1.16(\mathrm{t}, J=7.0 \mathrm{~Hz}, 3 \mathrm{H}), 1.02(\mathrm{tt}, J=2.5,15.0 \mathrm{~Hz}$, $1 \mathrm{H}) ;{ }^{13} \mathrm{C} \mathrm{NMR}\left(\mathrm{CDCl}_{3}, 75 \mathrm{MHz}\right) \delta 146.5(\mathrm{~d}, J=8.5 \mathrm{~Hz}), 141.6(\mathrm{~d}, J=4.3$ Hz), 139.8, 133.1, 128.8, 127.9, 65.4, 63.8 (d, $J=6.2 \mathrm{~Hz}), 62.4$ (d, $J=6.2$ $\mathrm{Hz}), 37.9,36.0,34.1,28.1,20.4,16.2 ;{ }^{31} \mathrm{P} \mathrm{NMR}\left(\mathrm{CDCl}_{3}, 282.216 \mathrm{MHz}\right) \delta$ 26.2; LRMS (CI) calculated for $\mathrm{C}_{17} \mathrm{H}_{25} \mathrm{O}_{6} \mathrm{PS}(\mathrm{M}+)$ 388, found $(\mathrm{M}+\mathrm{H}) 389$; HRMS (EI) calculated for $\mathrm{C}_{17} \mathrm{H}_{25} \mathrm{O}_{6} \mathrm{PS}(\mathrm{M}+\mathrm{H})$ 389.1188, found $(\mathrm{M}+\mathrm{H})$ 389.1183.

Preparation of Vinyl sulfone 32:

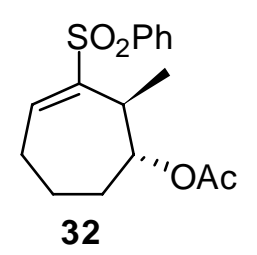

A solution of vinyl sulfone $28(0.266 \mathrm{~g}, 1 \mathrm{mmol})$ in $\mathrm{CH}_{2} \mathrm{Cl}_{2}(10 \mathrm{~mL})$ was cooled to $0^{\circ} \mathrm{C}$ for 30 minutes. Pyridine $(0.324 \mathrm{~mL}, 4 \mathrm{mmol})$ and DMAP (12 $\mathrm{mg}, 0.1 \mathrm{mmol}$ ) were added and the mixture was stirred for additional 10 minutes then acetic anhydride $(0.19 \mathrm{~mL}, 2 \mathrm{mmol})$ was added dropwise via syringe and the reaction was allowed to reach ambient temperature while stirring was continued for $3 \mathrm{~h}$. The reaction was checked for completion using TLC (50\% ethyl acetate/hexanes) then the reaction was washed with $5 \% \mathrm{HCl}$, aq. saturated $\mathrm{K}_{2} \mathrm{CO}_{3}$ and brine. The organic phase was dried over $\mathrm{Na}_{2} \mathrm{SO}_{4}$ then was concentrated via rotary evaporation to afford crude 32 as white needle crystals. Purification by flash column chromatography ( $40 \%$ ethyl acetate/hexanes) afforded 32 white needle crystals (249 mg, 81\%). Melting range $89.2-91.4^{\circ} \mathrm{C}$. TLC (40\% ethyl acetate/hexanes) $R_{f} 0.60 ;{ }^{1} \mathrm{H}$ $\operatorname{NMR}\left(\mathrm{CDCl}_{3}, 300 \mathrm{MHz}\right) \delta 7.83(\mathrm{dt}, J=1.5,6.9 \mathrm{~Hz}, 2 \mathrm{H}), 7.63(\mathrm{tt}, J=1.2$, 
$7.5 \mathrm{~Hz}, 1 \mathrm{H}), 7.58$ (t, $J=7.8 \mathrm{~Hz}, 2 \mathrm{H}), 7.36(\mathrm{dd}, J=3.6,9.3 \mathrm{~Hz}, 1 \mathrm{H}), 4.84$ (dt, $J=2.0,4.6 \mathrm{~Hz}, 1 \mathrm{H}), 3.00(\mathrm{dq}, J=6.8,6.8 \mathrm{~Hz}, 1 \mathrm{H}), 2.50(\mathrm{~m}, 1 \mathrm{H}), 2.28(\mathrm{~m}$, $1 \mathrm{H}), 1.80(\mathrm{~m}, 2 \mathrm{H}), 1.60(\mathrm{tt}, J=3.1,9.4 \mathrm{~Hz}, 2 \mathrm{H}), 1.57(\mathrm{~s}, 3 \mathrm{H}), 1.03(\mathrm{~d}, J=$ $7.5 \mathrm{~Hz}, 3 \mathrm{H}) ;{ }^{13} \mathrm{C} \mathrm{NMR}\left(\mathrm{CDCl}_{3}, 75 \mathrm{MHz}\right) \delta 169.2,143.9,141.8,139.1$, 132.9, 128.8, 128.2, 71.8, 36.3, 28.9, 27.7, 20.4, 19.2, 15.8; LRMS (EI) calculated for $\mathrm{C}_{16} \mathrm{H}_{20} \mathrm{O}_{4} \mathrm{~S}(\mathrm{M}+)$ 308, found 248 (M-HOAc); HRMS (EI) calculated for $\mathrm{C}_{16} \mathrm{H}_{20} \mathrm{O}_{4} \mathrm{~S}(\mathrm{M}+\mathrm{H}) 309.1161$, found 309.1157 .

Preparation of vinyl phosphonate 32a:

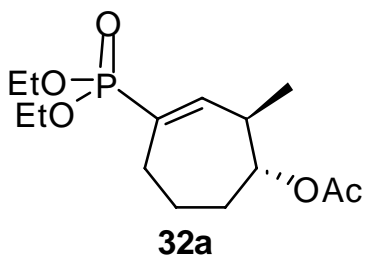

Following general procedure (B) and starting from vinyl sulfone 32 (0.3 g, $0.98 \mathrm{mmol}$ ), vinyl phosphonate 32a was received as a crude colorless oil. Purification by flash column chromatography (60\% ethylacetate/hexanes) afforded 32a as a colorless oil (188 mg, 63\%). TLC (80\% ethyl acetate/hexanes) $R_{f}$ 0.24. ${ }^{1} \mathrm{H} \mathrm{NMR}\left(\mathrm{CDCl}_{3}, 300 \mathrm{MHz}\right) \delta 6.52(\mathrm{dd}, J=1.5$, $23.8 \mathrm{~Hz}, 1 \mathrm{H}), 4.61$ (dt, $J=3.6,9.6 \mathrm{~Hz}, 1 \mathrm{H}), 4.08$ (m, 4H), 2.74 (br. m, 1H), $2.42(\mathrm{dt}, J=3.3,6.8 \mathrm{~Hz}, 1 \mathrm{H}), 2.20(\mathrm{~m}, 1 \mathrm{H}), 2.10(\mathrm{~m}, 1 \mathrm{H}), 2.05(\mathrm{~s}, 3 \mathrm{H}), 1.78$ $(\mathrm{m}, 2 \mathrm{H}), 1.48(\mathrm{~m}, 1 \mathrm{H}), 1.34(\mathrm{dt}, J=2.8,6.9 \mathrm{~Hz}, 6 \mathrm{H}), 1.13(\mathrm{~d}, J=6.8 \mathrm{~Hz}$, $3 \mathrm{H}) ;{ }^{13} \mathrm{C} \mathrm{NMR}\left(\mathrm{CDCl}_{3}, 75 \mathrm{MHz}\right) \delta 170.2,148.5(\mathrm{~d}, J=9.4 \mathrm{~Hz}), 74.8,63.5$ (d, $J=9.4 \mathrm{~Hz}), 39.3$ (d, $J=18.8 \mathrm{~Hz}), 36.2,27.3$ (d, $J=9.4 \mathrm{~Hz}), 24.1$ (d, $J=$ $1.9 \mathrm{~Hz}), 22.1(\mathrm{~d}, J=18.8 \mathrm{~Hz}), 17.6,16.4 ;{ }^{31} \mathrm{P} \mathrm{NMR}\left(\mathrm{CDCl}_{3}, 282.216 \mathrm{MHz}\right)$ 
$\delta$ 20.9; LRMS (CI) calculated for $\mathrm{C}_{14} \mathrm{H}_{25} \mathrm{O}_{5} \mathrm{P}(\mathrm{M}+\mathrm{H})$ 304, found 304; HRMS (EI) calculated for $\mathrm{C}_{14} \mathrm{H}_{25} \mathrm{O}_{5} \mathrm{P}(\mathrm{M}+)$ 304.1440, found 304.1433.

Preparation of Vinyl sulfone 33:

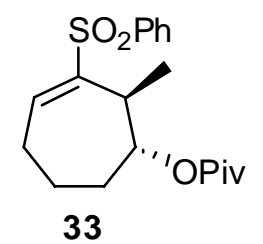

A solution of vinyl sulfone $28(0.273 \mathrm{~g}, 1.02 \mathrm{mmol})$ in 1,2-DCE $(9 \mathrm{~mL})$ was treated with pyridine $(0.33 \mathrm{~mL}, 4.08 \mathrm{mmol})$ and DMAP $(12 \mathrm{mg}, 0.1 \mathrm{mmol})$ and the mixture was stirred for 10 minutes at ambient temperature. Pivaloyl chloride $(0.25 \mathrm{~mL}, 2.04 \mathrm{mmol})$ was added dropwise via syringe and the reaction was refluxed for $6 \mathrm{~h}$. The reaction was checked for completion using TLC (50\% ethyl acetate/hexanes) then the reaction was washed with $5 \%$ $\mathrm{HCl}$, aq. saturated $\mathrm{K}_{2} \mathrm{CO}_{3}$ and brine. The organic phase was dried over $\mathrm{Na}_{2} \mathrm{SO}_{4}$ then was concentrated via rotary evaporation to afford crude 33 as white needle crystals. Purification by flash column chromatography ( $40 \%$ ethyl acetate/hexanes) afforded 33 white needle crystals (294 mg, 82\%). Melting range $93.2-95.3^{\circ} \mathrm{C}$. TLC (40\% ethyl acetate/hexanes) $R_{f} \quad 0.77 ;{ }^{1} \mathrm{H}$ $\operatorname{NMR}\left(\mathrm{CDCl}_{3}, 300 \mathrm{MHz}\right) \delta 7.85(\mathrm{~d}, J=7.8 \mathrm{~Hz}, 2 \mathrm{H}), 7.57(\mathrm{dt}, J=2.1,8.7$ $\mathrm{Hz}, 1 \mathrm{H}), 7.50(\mathrm{dt}, J=1.5,8.1 \mathrm{~Hz}, 2 \mathrm{H}), 7.34(\mathrm{dt}, J=3.0,9.0 \mathrm{~Hz}, 1 \mathrm{H}), 4.87$ (m, $J=2.4 \mathrm{~Hz}, 1 \mathrm{H}), 3.09$ (p; $J=6.6 \mathrm{~Hz}, 1 \mathrm{H}), 2.52$ (ddt, $J=3.9,7.8,12.9$, 1H), $2.25(\mathrm{~m}, 1 \mathrm{H}), 2.02(\mathrm{~m}, 2 \mathrm{H}), 1.88(\mathrm{~m}, 1 \mathrm{H}), 1.6(\mathrm{~m}, 2 \mathrm{H}), 1.06(\mathrm{~d}, J=2.1$ $\mathrm{Hz}, 9 \mathrm{H}), 0.87(\mathrm{dd}, J=2.1,7.2 \mathrm{~Hz}, 3 \mathrm{H}) ;{ }^{13} \mathrm{C} \mathrm{NMR}\left(\mathrm{CDCl}_{3}, 75 \mathrm{MHz}\right) \delta 169.2$, $143.9,141.8,139.1,132.9,128.8,128.2,71.8,36.3,28.9,27.7,20.4,19.2$, 15.8; LRMS (EI) calculated for $\mathrm{C}_{16} \mathrm{H}_{20} \mathrm{O}_{4} \mathrm{~S}(\mathrm{M}+)$ 308, found 248 (M-HOAc); HRMS (EI) calculated for $\mathrm{C}_{16} \mathrm{H}_{20} \mathrm{O}_{4} \mathrm{~S}(\mathrm{M}+\mathrm{H})$ 309.1161, found 309.1157 . 
Preparation of vinyl phosphonate 33a:

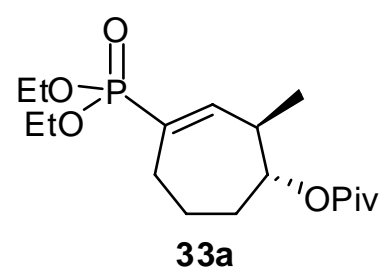

KHMDS (203 $\mu \mathrm{L}, 0.19 \mathrm{mmol}, 0.9 \mathrm{M}$ in THF) was dropwise added to a clear colorless stirred solution of $33(45 \mathrm{mg}, 0.13 \mathrm{mmol})$ in THF $(0.5 \mathrm{~mL})$ at $-6{ }^{\circ} \mathrm{C}$ to produce a deep yellow solution. After 5 minutes, the ice bath was removed and the reaction was run at room temperature for $12 \mathrm{~h},{ }^{5}$ then quenched with wet ether and transferred to a separatory funnel. Saturated $\mathrm{NaHCO}_{3}$ was added and the products were extracted with ether $(2 \times 20$ $\mathrm{mL}$ ). The combined ethereal layers were dried with sodium sulfate, and concentrated by rotary evaporation to give a light yellow oil that was purified by column chromatography (Hexanes: EtOAc $=1: 1 \rightarrow 1: 2$ ) to give 34 $\mathrm{mg}(77 \%)$ of 33a as a colorless oil. ${ }^{1} \mathrm{H} \mathrm{NMR}\left(\mathrm{CDCl}_{3}, 300 \mathrm{MHz}\right) \delta 6.53(\mathrm{dd}$, $J=4.0,19.6 \mathrm{~Hz}, 1 \mathrm{H}), 4.55(\mathrm{td}, J=2.4,7.2 \mathrm{~Hz}, 1 \mathrm{H}), 4.04(\mathrm{~m}, 4 \mathrm{H}), 2.76(\mathrm{~m}$, 1H), $2.20(\mathrm{~m}, 1 \mathrm{H}), 2.07(\mathrm{~m}, 1 \mathrm{H}), 1.74(\mathrm{~m}, 2 \mathrm{H}), 1.47(\mathrm{~m}, 1 \mathrm{H}), 1.31(\mathrm{q}, J=6.0$ $\mathrm{Hz}, 6 \mathrm{H}), 1.18(\mathrm{~s}, 9 \mathrm{H}), 1.11(\mathrm{~d}, J=5.6 \mathrm{~Hz}, 3 \mathrm{H}) ;{ }^{13} \mathrm{C} \mathrm{NMR}\left(\mathrm{CDCl}_{3}, 75 \mathrm{MHz}\right)$ $\delta 177.5,149.1(\mathrm{~d}, J=10.0 \mathrm{~Hz}), 133.5(\mathrm{~d}, J=170.0 \mathrm{~Hz}), 73.4,61.7(\mathrm{~d}, J=$ $5.0 \mathrm{~Hz}), 61.6(\mathrm{~d}, J=5.0 \mathrm{~Hz}), 39.3(\mathrm{~d}, J=20.0 \mathrm{~Hz}), 39.0,35.9,27.3(\mathrm{~d}, J=$ $9.0 \mathrm{~Hz}), 27.1,24.0(\mathrm{~d}, J=7.0 \mathrm{~Hz}), 17.5,16.3 ;{ }^{31} \mathrm{P} \mathrm{NMR}\left(\mathrm{CDCl}_{3}, 282.216\right.$ MHz) $\delta 20.9$; HRMS (EI) calculated for $\mathrm{C}_{17} \mathrm{H}_{31} \mathrm{O}_{5} \mathrm{P}(\mathrm{M}+)$ 346.1909, found 346.1907 .

\footnotetext{
${ }^{5}$ After about 30 min at room temperature, the reaction became a heavy white suspension.
} 
Preparation of vinyl sulfone 34:

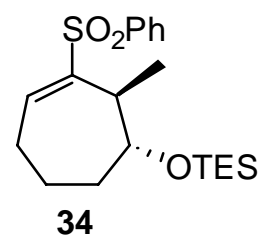

A solution of vinyl sulfone $28(0.68 \mathrm{~g}, 2.55 \mathrm{mmol})$ in 1,2-DCE (1,2dichloroethane, $6.5 \mathrm{~mL})$ was treated with TESCl $(0.46 \mathrm{~g}, 3 \mathrm{mmol})$ and imidazole $(0.317 \mathrm{~g}, 4.6 \mathrm{mmol})$ then the mixture was refluxed for $6 \mathrm{~h}$. The reaction mixture was then diluted with 1,2-DCE $(6 \mathrm{~mL})$, washed with $5 \%$ $\mathrm{HCl}$, brine and then dried over $\mathrm{Na}_{2} \mathrm{SO}_{4}$. Concentration via rotary evaporation afforded 34 as a crude white solid. Purification by flash column chromatography (30\% ethyl acetate/hexanes) afforded pure 34 as a white crystalline solid $(0.93 \mathrm{~g}, 96 \%)$. Melting range $73.5-74.0^{\circ} \mathrm{C}$. TLC (30\% ethyl acetate/hexanes) $R_{f} 0.56 ;{ }^{1} \mathrm{H}$ NMR $\left(\mathrm{CDCl}_{3}, 300 \mathrm{MHz}\right) \delta 7.82(\mathrm{~m}, 2 \mathrm{H}), 7.53$ (tt, $J=1.2,6.0 \mathrm{~Hz}, 1 \mathrm{H}), 7.45(\mathrm{tt}, J=0.9,6.0 \mathrm{~Hz}, 2 \mathrm{H}), 7.28$ (dd, $J=2.1,6.3$ $\mathrm{Hz}, 1 \mathrm{H}), 3.74(\mathrm{t}, J=3.6 \mathrm{~Hz}, 1 \mathrm{H}), 2.77(\mathrm{~m}, 1 \mathrm{H}), 2.45(\mathrm{~m}, 1 \mathrm{H}), 2.17(\mathrm{tt}, J=$ 2.4, $9.9 \mathrm{~Hz}, 1 \mathrm{H}), 1.76(\mathrm{~m}, 2 \mathrm{H}), 1.45(\mathrm{~m}, 2 \mathrm{H}), 1.03(\mathrm{~d}, J=5.1 \mathrm{~Hz}, 3 \mathrm{H}), 0.74$ $(\mathrm{t}, J=6.0 \mathrm{~Hz}, 9 \mathrm{H}), 0.26(\mathrm{q}, J=6.0 \mathrm{~Hz}, 6 \mathrm{H}) ;{ }^{13} \mathrm{C} \mathrm{NMR}\left(\mathrm{CDCl}_{3}, 75 \mathrm{MHz}\right) \delta$ $144.3,141.8,139.8,132.6,128.7,128.1,70.5,40.7,32.3,28.1,18.4,16.5$, 6.7, 4.3; LRMS (CI) calculated for $\mathrm{C}_{20} \mathrm{H}_{32} \mathrm{O}_{3} \mathrm{SSi}(\mathrm{M}+\mathrm{H})$ 381, found 381; HRMS (EI) calculated for $\mathrm{C}_{20} \mathrm{H}_{32} \mathrm{O}_{3} \mathrm{SSi}(\mathrm{M}+\mathrm{H})$ 381.1920, found 381.1921 . 
Preparation of vinyl phosphonate 34a:

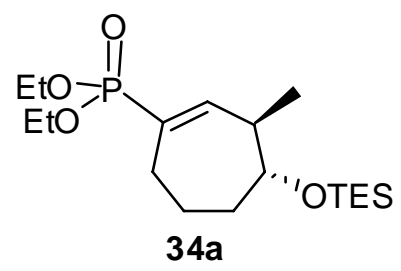

$\mathrm{KOBu}^{\mathrm{t}}(186 \mu \mathrm{L}, 0.19 \mathrm{mmol}, 1 \mathrm{M}$ in THF) was dropwise added to a clear colorless stirred solution of $34(51 \mathrm{mg}, 0.132 \mathrm{mmol})$ in THF $(1.3 \mathrm{~mL})$ at -15 ${ }^{\circ} \mathrm{C}$ to produce a light yellow solution. After 5 minutes, the ice bath was removed and the reaction was run at room temperature for $12 \mathrm{~h},{ }^{6}$ then quenched with wet ether and transferred to a separatory funnel. Saturated $\mathrm{NaHCO}_{3}$ was added and the products were extracted with ether $(2 \times 20 \mathrm{~mL})$. The combined ethereal layers were dried with sodium sulfate, and concentrated by rotary evaporation to give a light yellow oil that was purified by column chromatography (Hexanes: EtOAc $=3: 2 \rightarrow 1: 1$ ) to give 38 $\mathrm{mg}(76 \%)$ of $34 \mathrm{a}$ as a colorless oil. ${ }^{1} \mathrm{H}$ NMR $\left(\mathrm{CDCl}_{3}, 300 \mathrm{MHz}\right) \delta 6.53(\mathrm{ddd}$, $J=1.6,5.2,24.0 \mathrm{~Hz}, 1 \mathrm{H}), 4.05(\mathrm{~m}, 4 \mathrm{H}), 3.33(\mathrm{td}, J=3.2,9.2 \mathrm{~Hz}, 1 \mathrm{H}), 2.57$ (m, 1H), $2.34(\mathrm{~m}, 1 \mathrm{H}), 2.18(\mathrm{~m}, 1 \mathrm{H}), 2.07(\mathrm{~m}, 1 \mathrm{H}), 1.79-1.66(\mathrm{~m}, 2 \mathrm{H}), 1.34$ (m, 1H), 1.40 (obscured multiplet, 1H), 1.31 (q, $J=6.8 \mathrm{~Hz}, 6 \mathrm{H}), 1.14$ (d, $J=$ $7.2 \mathrm{~Hz}, 3 \mathrm{H}), 0.94(\mathrm{t}, J=8.0 \mathrm{~Hz}, 9 \mathrm{H}), 0.58$ (q, $J=8.0 \mathrm{~Hz}, 6 \mathrm{H}) ;{ }^{13} \mathrm{C} \mathrm{NMR}$ $\left(\mathrm{CDCl}_{3}, 75 \mathrm{MHz}\right) \delta 150.8(\mathrm{~d}, J=10.7 \mathrm{~Hz}), 132.7(\mathrm{~d}, J=140.0 \mathrm{~Hz}), 72.3$, $61.5(\mathrm{~d}, J=5.4 \mathrm{~Hz}), 42.2(\mathrm{~d}, J=20.0 \mathrm{~Hz}), 40.6,27.4(\mathrm{~d}, J=10.0 \mathrm{~Hz}), 24.6$ $(\mathrm{d}, J=10.0 \mathrm{~Hz}), 18.2(\mathrm{~d}, J=18.8 \mathrm{~Hz}), 16.4,6.9,4.9 ;{ }^{31} \mathrm{P} \mathrm{NMR}\left(\mathrm{CDCl}_{3}\right.$, $282.216 \mathrm{MHz}) \delta 22.5$; HRMS (EI) calculated for $\mathrm{C}_{18} \mathrm{H}_{37} \mathrm{O}_{4} \mathrm{PSi}(\mathrm{M}+)$ 376.2199 , found 376.2195 .

\footnotetext{
${ }^{6}$ After about $30 \mathrm{~min}$ at room temperature, the reaction became a heavy white suspension.
} 
Preparation of vinyl sulfone 35:

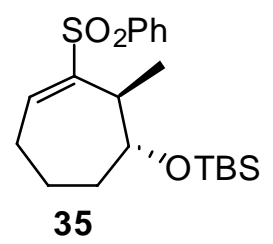

A solution of vinyl sulfone $28(0.68 \mathrm{~g}, 2.55 \mathrm{mmol})$ in 1,2-DCE (1,2dichloroethane, $6.5 \mathrm{~mL})$ was treated with $\mathrm{TBSCl}(0.46 \mathrm{~g}, 3 \mathrm{mmol})$ and imidazole $(0.317 \mathrm{~g}, 4.6 \mathrm{mmol})$ then the mixture was refluxed for $6 \mathrm{~h}$. The reaction mixture was then diluted with 1,2-DCE $(6 \mathrm{~mL})$, washed with 5\% $\mathrm{HCl}$, brine and then dried over $\mathrm{Na}_{2} \mathrm{SO}_{4}$. Concentration via rotary evaporation afforded 35 as a crude white solid. Purification by flash column chromatography (30\% ethyl acetate/hexanes) afforded pure 35 as a white crystalline solid $(0.89 \mathrm{~g}, 92 \%)$. Melting range $75.5-76.0^{\circ} \mathrm{C}$. TLC (30\% ethyl acetate/hexanes) $R_{f} 0.56 ;{ }^{1} \mathrm{H} \mathrm{NMR}\left(\mathrm{CDCl}_{3}, 300 \mathrm{MHz}\right) \delta 7.86(\mathrm{dt}, J=1.5$, $6.9 \mathrm{~Hz}, 2 \mathrm{H}), 7.56(\mathrm{tt}, J=1.2,7.5 \mathrm{~Hz}, 1 \mathrm{H}), 7.50(\mathrm{t}, J=7.8 \mathrm{~Hz}, 2 \mathrm{H}), 7.36(\mathrm{dd}$, $J=3.9,8.8 \mathrm{~Hz}, 1 \mathrm{H}), 3.80(\mathrm{~m}, 1 \mathrm{H}), 2.87$ (dq, $J=7.4,7.5 \mathrm{~Hz}, 1 \mathrm{H}), 2.51$ (m, 1H), $2.20(\mathrm{~m}, 1 \mathrm{H}), 1.82(\mathrm{~m}, 3 \mathrm{H}), 1.52(\mathrm{tq}, J=1.7,5.0 \mathrm{~Hz}, 1 \mathrm{H}), 0.98(\mathrm{~d}, J=$ $6.5 \mathrm{~Hz}, 3 \mathrm{H}), 0.74(\mathrm{~s}, 9 \mathrm{H}),-0.13(\mathrm{~s}, 3 \mathrm{H}),-0.20(\mathrm{~s}, 3 \mathrm{H}) ;{ }^{13} \mathrm{C} \mathrm{NMR}\left(\mathrm{CDCl}_{3}, 75\right.$ $\mathrm{MHz}) \delta 144.5,141.9,140.1,132.6,128.8,128.2,71.1,40.2,32.1,28.1$, 25.9, 18.9, 18.2, 16.4, -5.2, -4.7; LRMS (CI) calculated for $\mathrm{C}_{20} \mathrm{H}_{32} \mathrm{O}_{3} \mathrm{SSi}$ $(\mathrm{M}+\mathrm{H})$ 381, found 381; HRMS (EI) calculated for $\mathrm{C}_{20} \mathrm{H}_{32} \mathrm{O}_{3} \mathrm{SSi}(\mathrm{M}+\mathrm{H})$ 381.1920, found 381.1921 . 
Preparation of vinyl phosphonate 35a:

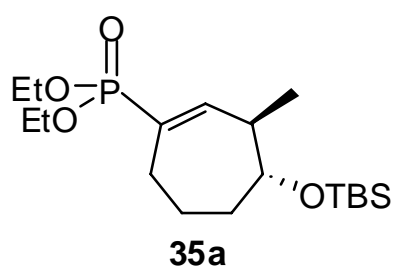

Following general procedure (A) and starting from vinyl sulfone 35 (300 mg, $0.8 \mathrm{mmol}$ ), vinyl phosphonate 35a was received as a crude colorless oil. Purification by flash column chromatography (50\% ethylacetate/hexanes) afforded 35a as a colorless oil (0.256 g, 85\%). TLC (60\% ethyl acetate/hexanes) $R_{f}$ 0.37. ${ }^{1} \mathrm{H} \mathrm{NMR}\left(\mathrm{CDCl}_{3}, 300 \mathrm{MHz}\right) \delta 6.48$ (ddd, $J=5.4$, 17.4, $24.0 \mathrm{~Hz}, 1 \mathrm{H}), 3.99(\mathrm{~m}, 4 \mathrm{H}), 3.26$ (dt, $J=2.6,8.4 \mathrm{~Hz}, 1 \mathrm{H}), 2.53$ (m, 1H), $2.28(\mathrm{~m}, 1 \mathrm{H}), 2.15(\mathrm{~m}, 1 \mathrm{H}), 2.01(\mathrm{~m}, 1 \mathrm{H}), 1.68(\mathrm{~m}, 2 \mathrm{H}), 1.34(\mathrm{~m}, 1 \mathrm{H})$, $1.25(\mathrm{dt}, J=4.8,6.9 \mathrm{~Hz}, 6 \mathrm{H}), 1.06(\mathrm{~d}, J=6.9 \mathrm{~Hz}, 3 \mathrm{H}), 0.80(\mathrm{~s}, 9 \mathrm{H}), 0.00$ (s, $6 \mathrm{H}) ;{ }^{13} \mathrm{C} \mathrm{NMR}\left(\mathrm{CDCl}_{3}, 75 \mathrm{MHz}\right) \delta 150.8(\mathrm{~d}, J=10.7 \mathrm{~Hz}), 132.5(\mathrm{~d}, J=$ $171.4 \mathrm{~Hz}), 72.2,62.4$ (d, $J=5.4 \mathrm{~Hz}), 44.1$ (d, $J=25.0 \mathrm{~Hz}), 40.2,27.2$ (d, $J=$ $12.5 \mathrm{~Hz}), 24.5$ (d, $J=6.3 \mathrm{~Hz}), 18.1$ (d, $J=18.8 \mathrm{~Hz}), 16.2 ;{ }^{31} \mathrm{P} \mathrm{NMR}\left(\mathrm{CDCl}_{3}\right.$, $282.216 \mathrm{MHz}) \delta 21.9$; LRMS (CI) calculated for $\mathrm{C}_{18} \mathrm{H}_{37} \mathrm{O}_{4} \mathrm{PSi}(\mathrm{M}+\mathrm{H}) 377$, found 377; HRMS (EI) calculated for $\mathrm{C}_{18} \mathrm{H}_{37} \mathrm{O}_{4} \mathrm{PSi}(\mathrm{M}+$ ) 376.2199, found 376.2195 . 
Preparation of vinyl phosphonate 36a:

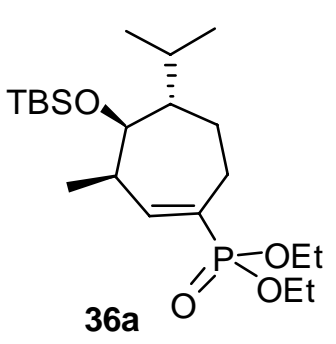

Following general procedure (B) and starting from vinyl sulfone 36 (80 mg, 0.19 mmol), vinyl phosphonate 36a was received as a crude colorless oil. Purification by flash column chromatography (20\% ethylacetate/hexanes) afforded 36a as a colorless oil (62 mg, 78\%). TLC (50\% ethyl acetate/hexanes) $R_{f}$ 0.43. ${ }^{1} \mathrm{H}$ NMR $\left(\mathrm{CDCl}_{3}, 300 \mathrm{MHz}\right) \delta 6.34(\mathrm{~d}, J=25.1$ Hz, 1H), 4.03 (obscured multiplet, 4H), 3.77 (ap. s, 1H), 2.70 (br. m, 1H), $2.28(\mathrm{~m}, 2 \mathrm{H}), 1.80(\mathrm{~m}, 2 \mathrm{H}), 1.51(\mathrm{~m}, 2 \mathrm{H}), 1.30(\mathrm{t}, J=6.9 \mathrm{~Hz}, 6 \mathrm{H}), 1.18(\mathrm{~d}, J$ $=7.4 \mathrm{~Hz}, 3 \mathrm{H}), 0.94(\mathrm{~d}, J=6.7 \mathrm{~Hz}, 3 \mathrm{H}), 0.90(\mathrm{~d}, J=6.7 \mathrm{~Hz}, 3 \mathrm{H}), 0.86(\mathrm{~s}$, 9H), $0.31(\mathrm{~d}, J=1.7 \mathrm{~Hz}, 6 \mathrm{H}) ;{ }^{13} \mathrm{C} \mathrm{NMR}\left(\mathrm{CDCl}_{3}, 75 \mathrm{MHz}\right) \delta 150.1(\mathrm{~d}, J=$ $9.2 \mathrm{~Hz}), 130.4(\mathrm{~d}, J=175.0 \mathrm{~Hz}), 75.9,61.4(\mathrm{dd}, J=6.3,3.8 \mathrm{~Hz}), 50.9,36.4$ (d, $J=20.4 \mathrm{~Hz}), 27.8,25.8,24.2(\mathrm{~d}, J=9.0 \mathrm{~Hz}), 23.1(\mathrm{~d}, J=7.9 \mathrm{~Hz}), 21.2$, 20.2, 19.2, 18.1, 16.4 (d, $J=7.3 \mathrm{~Hz}),-4.3,-4.7 ;{ }^{31} \mathrm{P}$ NMR $\left(\mathrm{CDCl}_{3}, 282.216\right.$ $\mathrm{MHz}) \delta 22.4$; LRMS (CI) calculated for $\mathrm{C}_{21} \mathrm{H}_{43} \mathrm{O}_{4} \mathrm{PSi}(\mathrm{M}+)$ 418, found 419.2 $(\mathrm{M}+\mathrm{H})$. 


\section{Mechansims}

Proposed Mechanism for conversion of $\mathbf{2 8}$ to $\mathbf{2 8 a}$ :

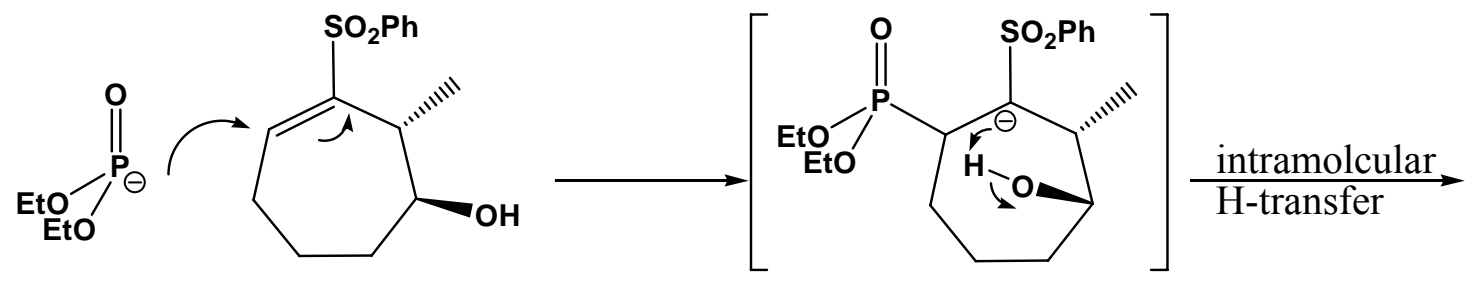

28

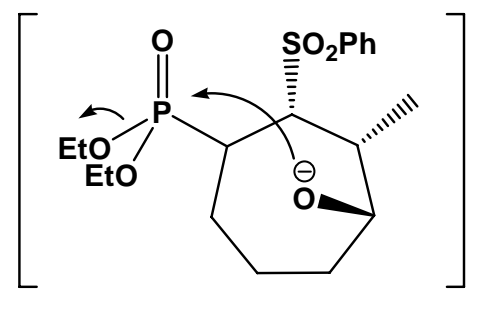

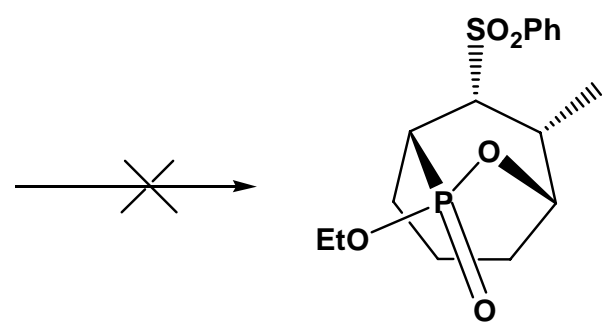

Not Observed

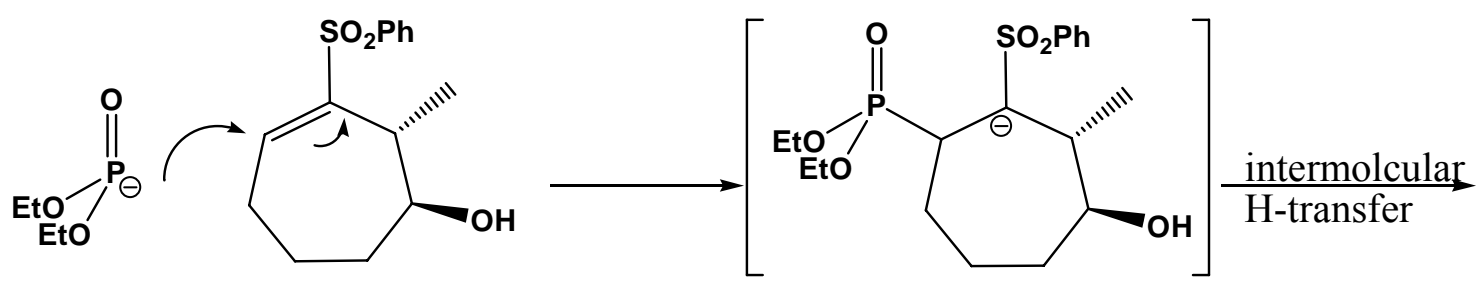

28

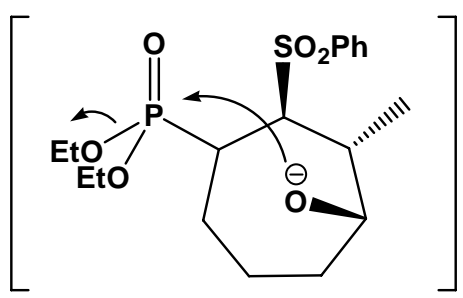

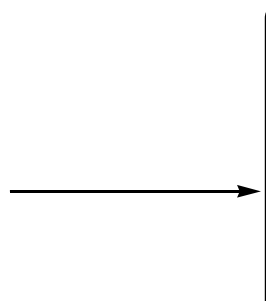

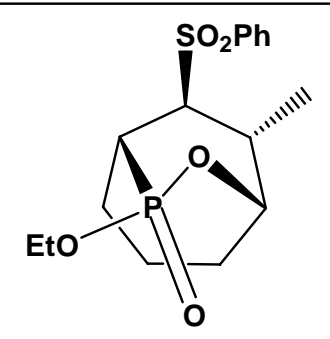

28a Observed 
Proposed Mechanism for conversion of $\mathbf{2 7}$ to $\mathbf{2 7} \mathbf{b}$ :<smiles>CCO[R5](=O)(OCC)C1C(CP(=O)(OCC)OCC)CCCC1P(=O)(OCC)OCC</smiles><smiles>CCOP(=O)(OCC)C1CCC(CC[18O])C=C1S(=O)(=O)Oc1ccccc1</smiles>

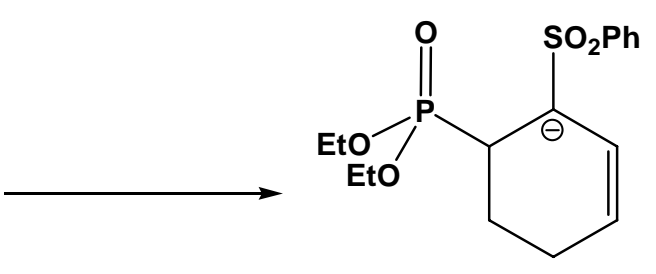

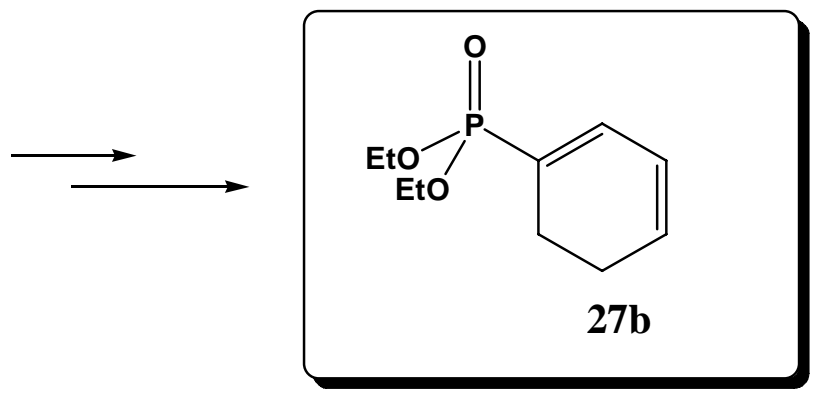




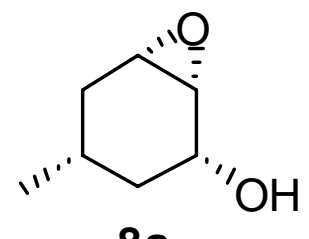

$8 a$

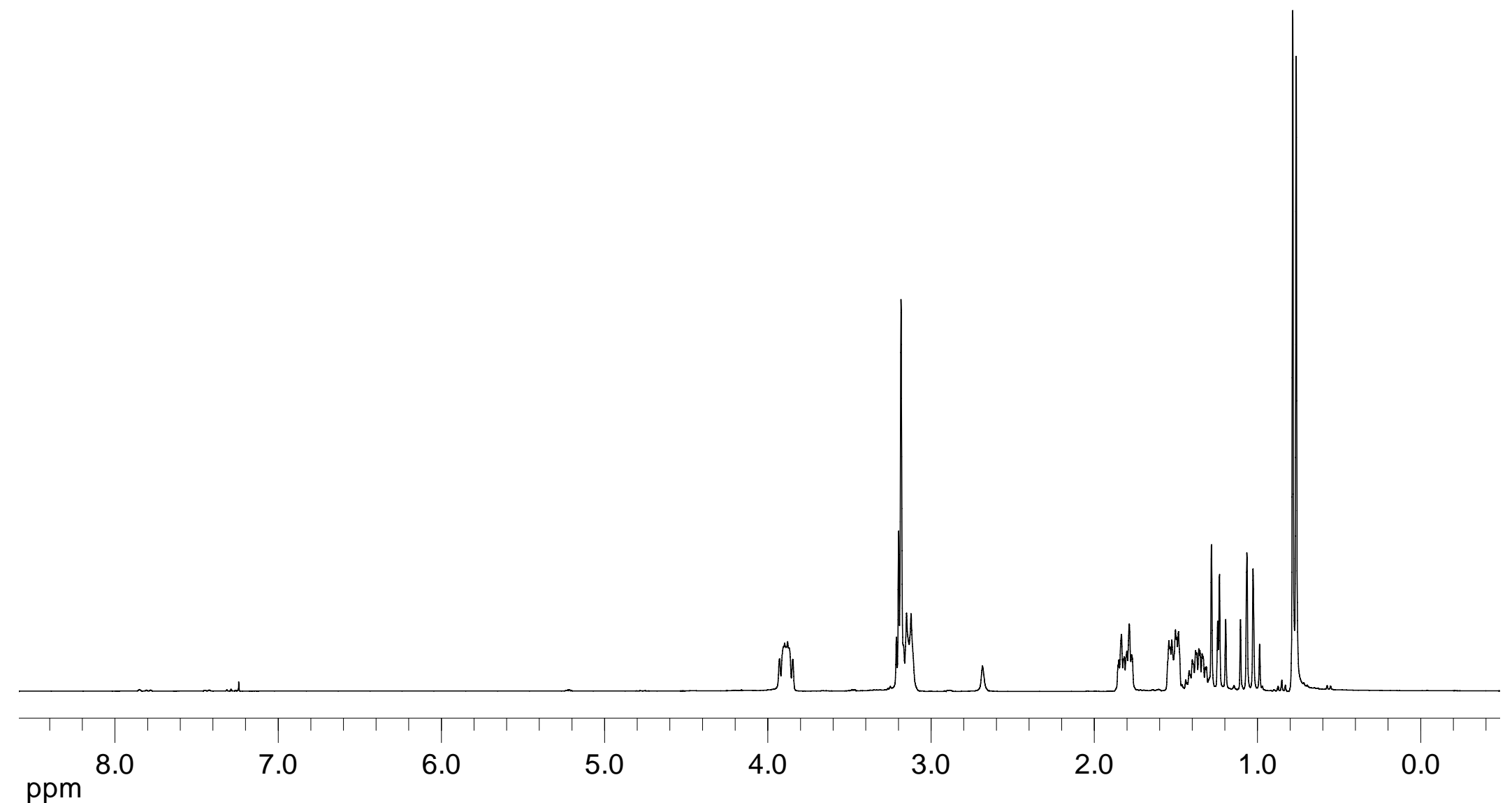



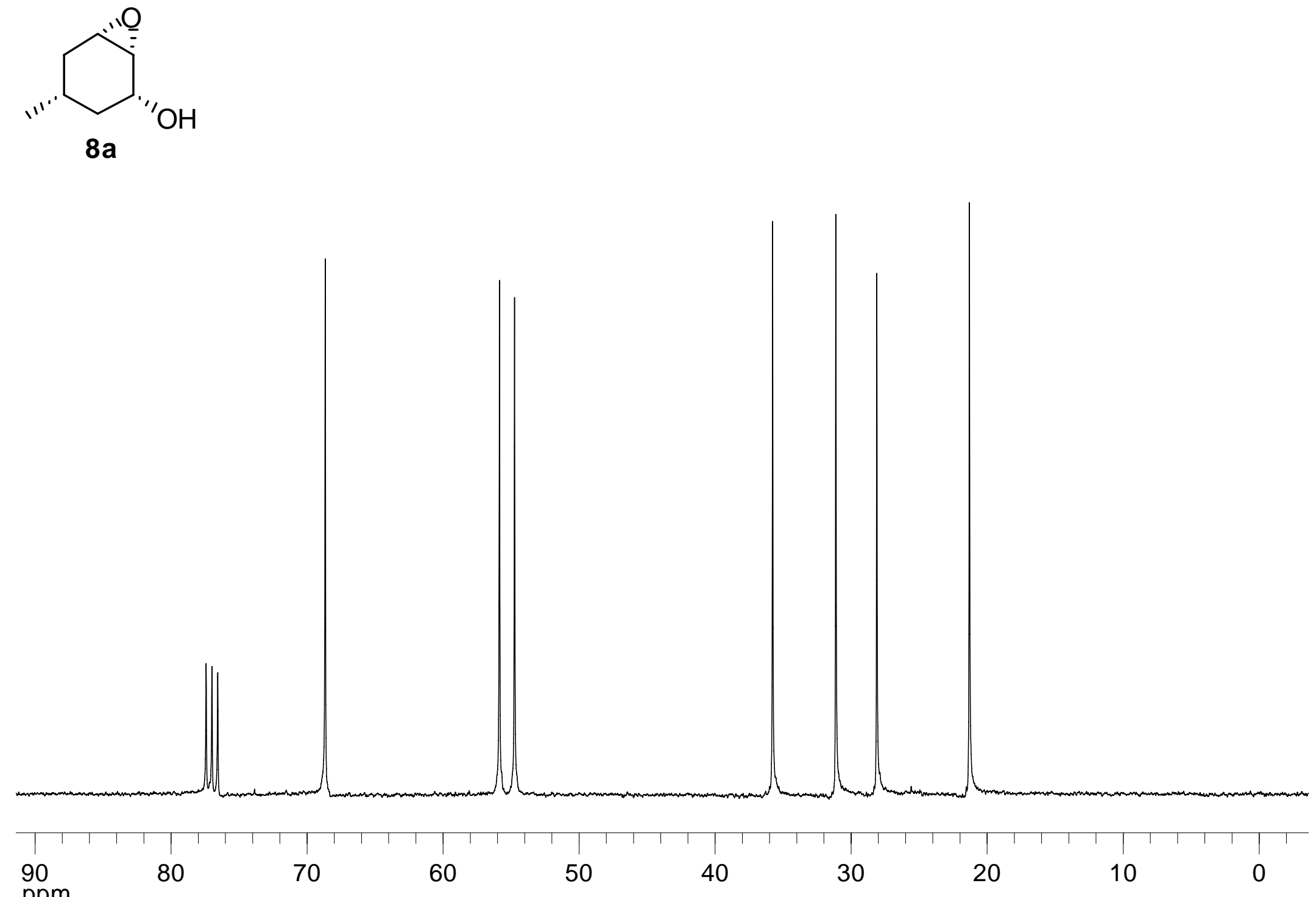

ppm 

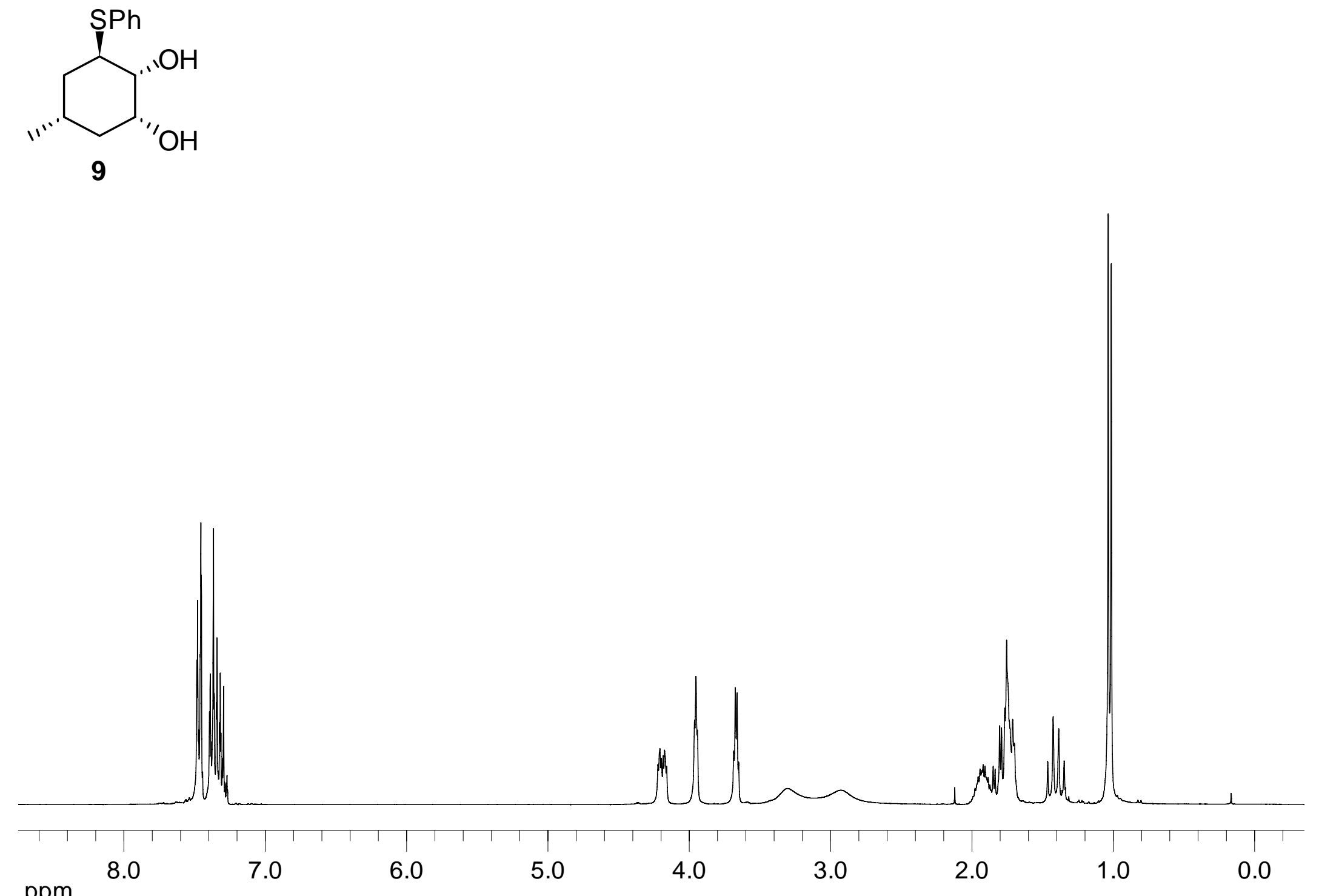

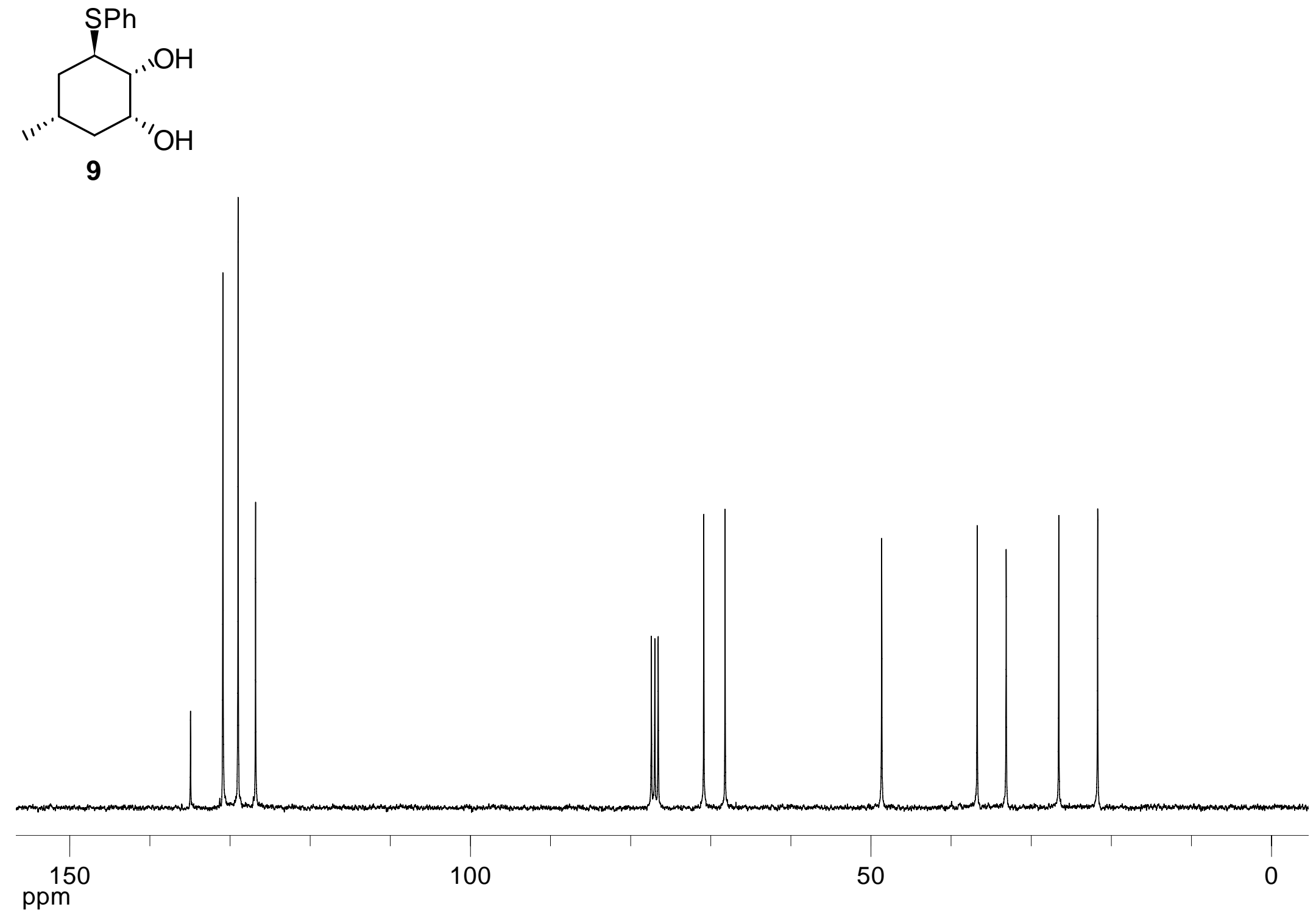

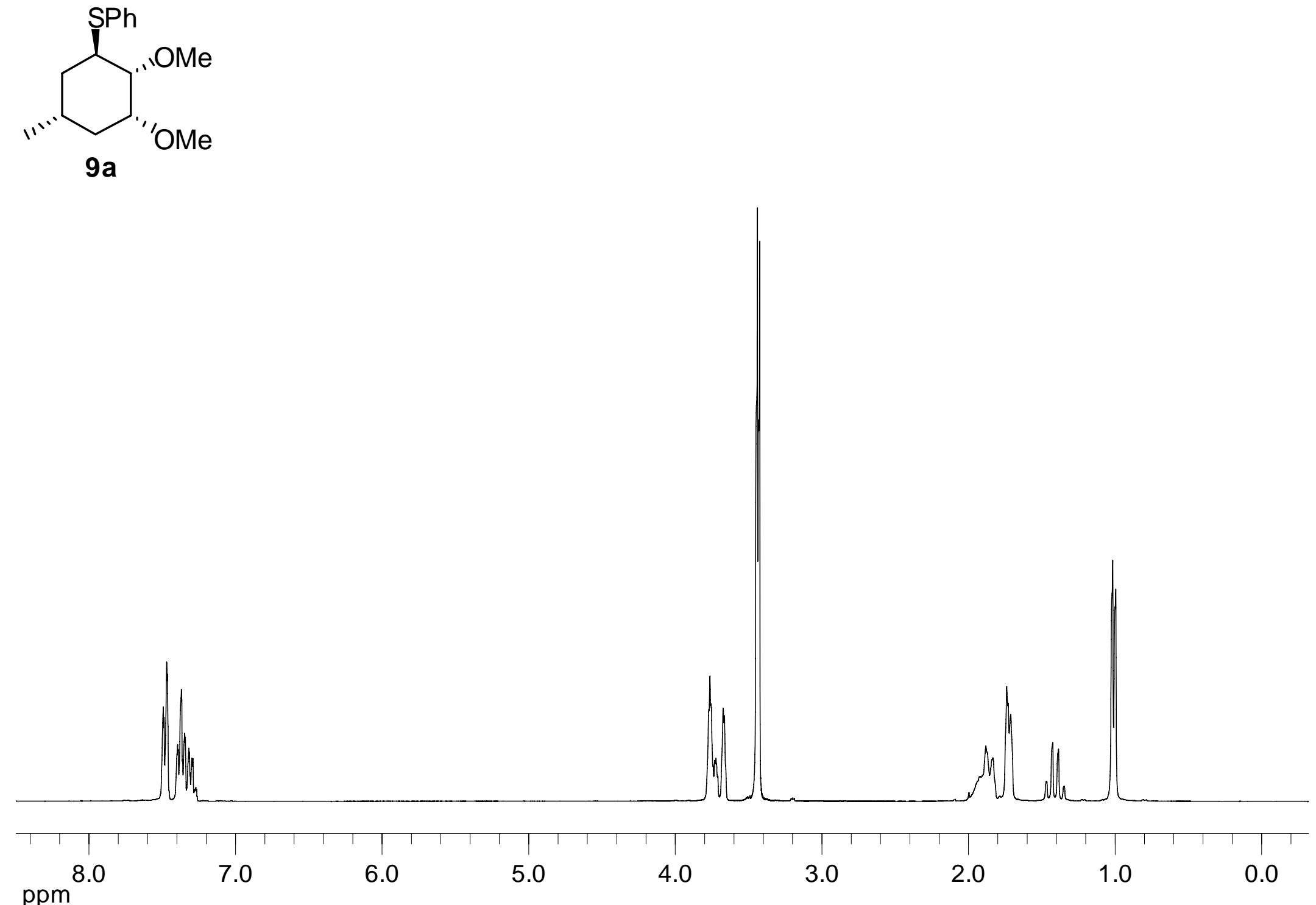

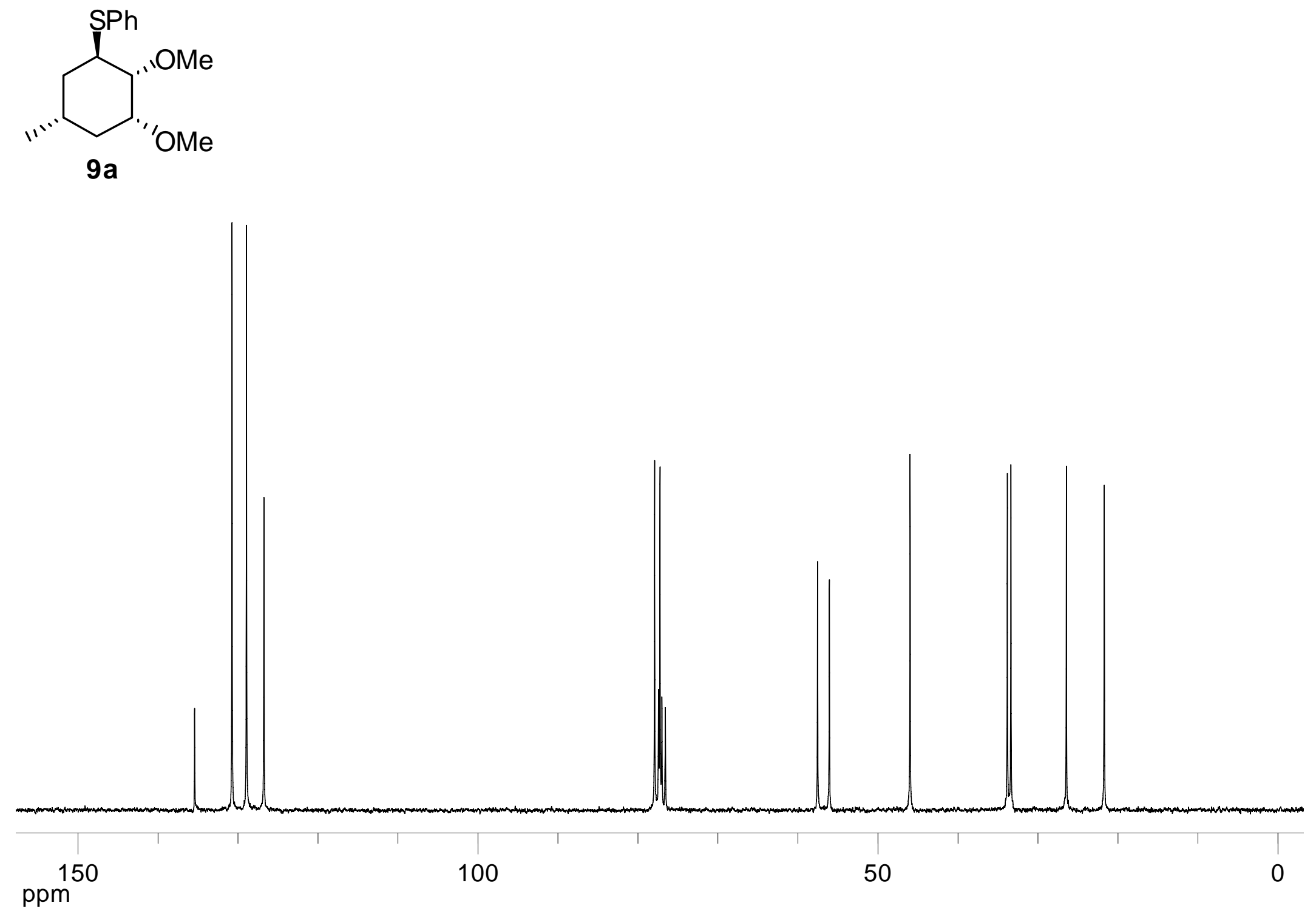

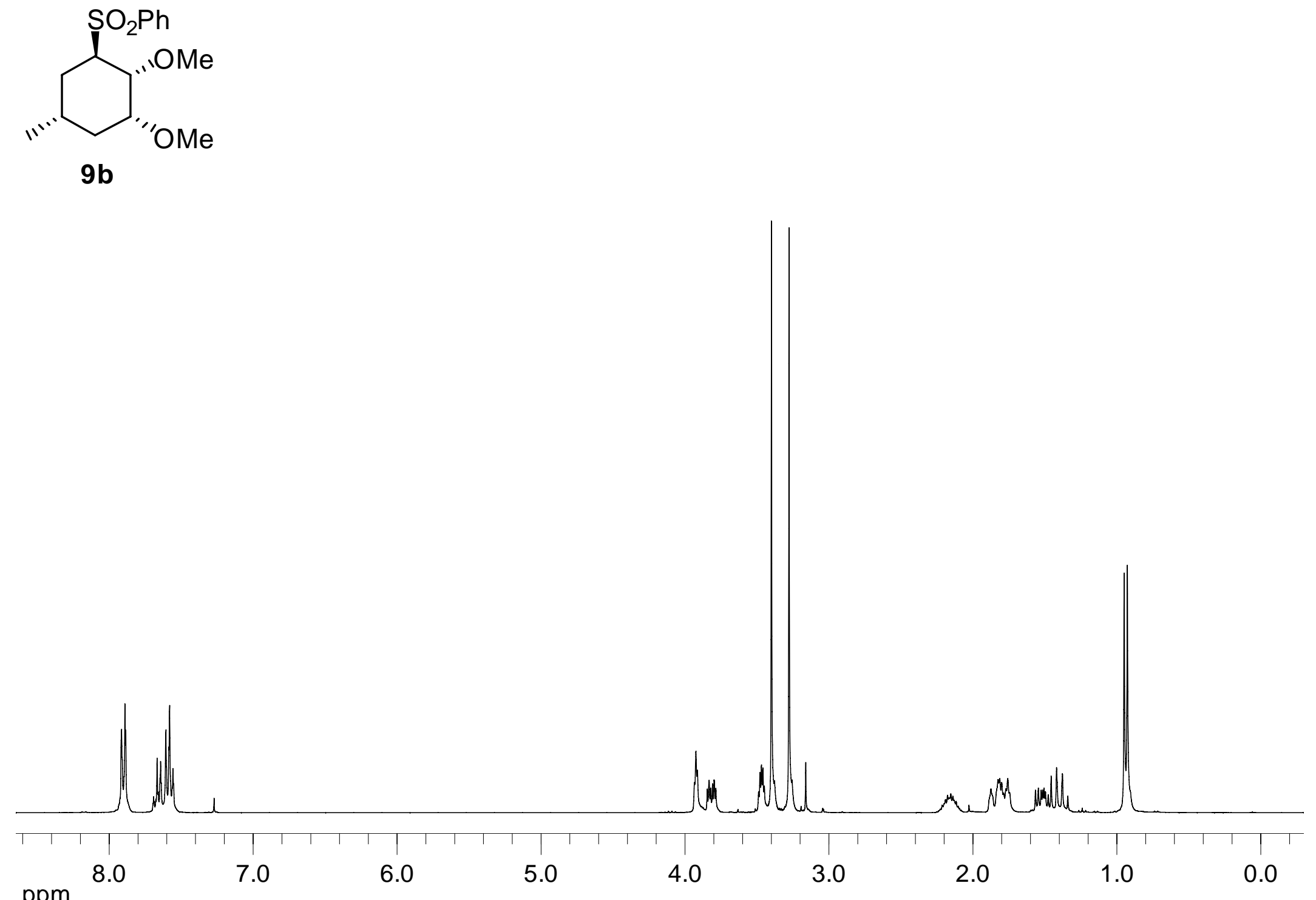


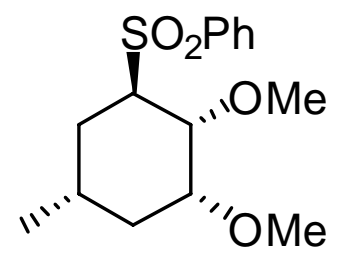

9b

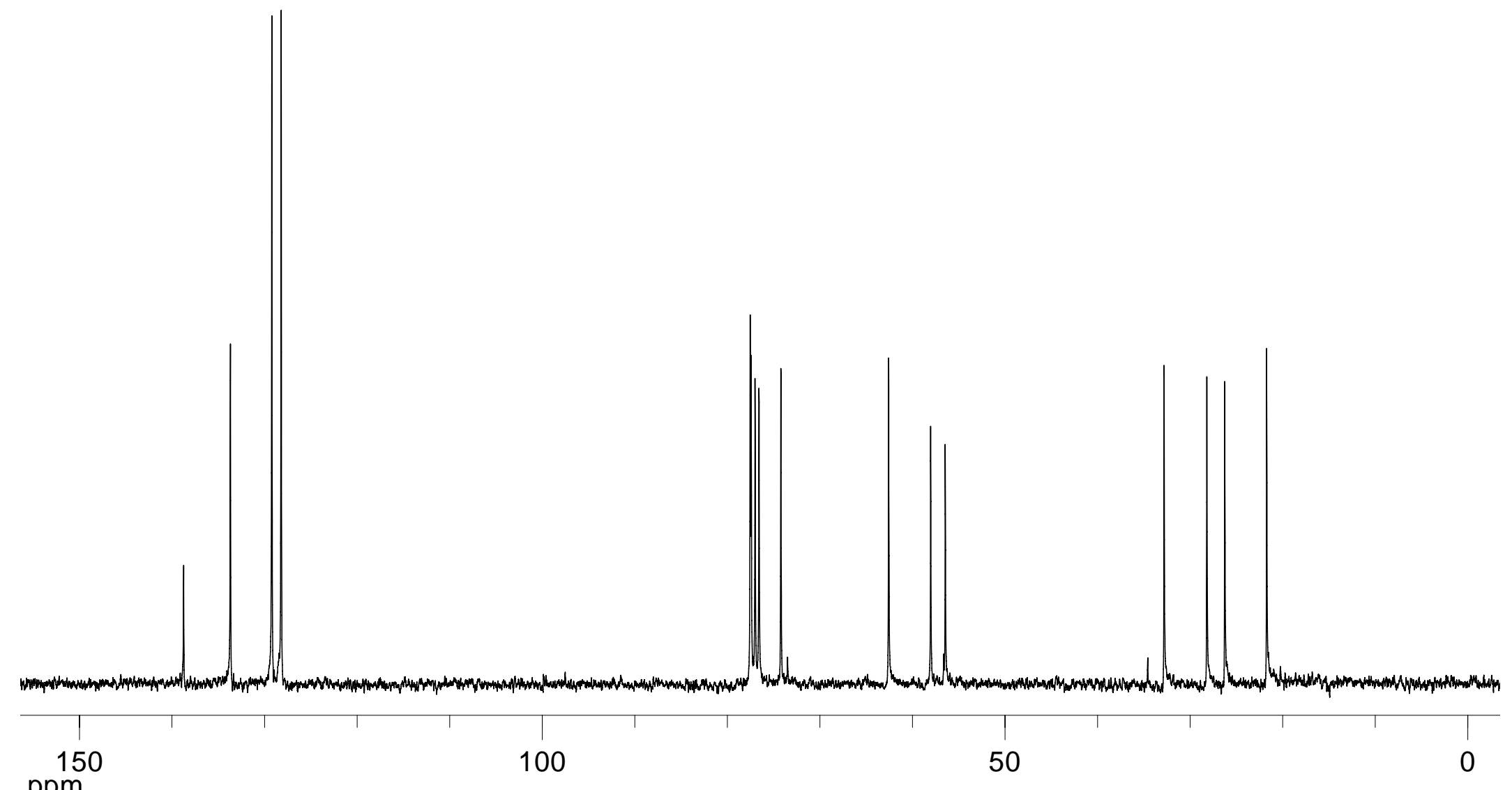



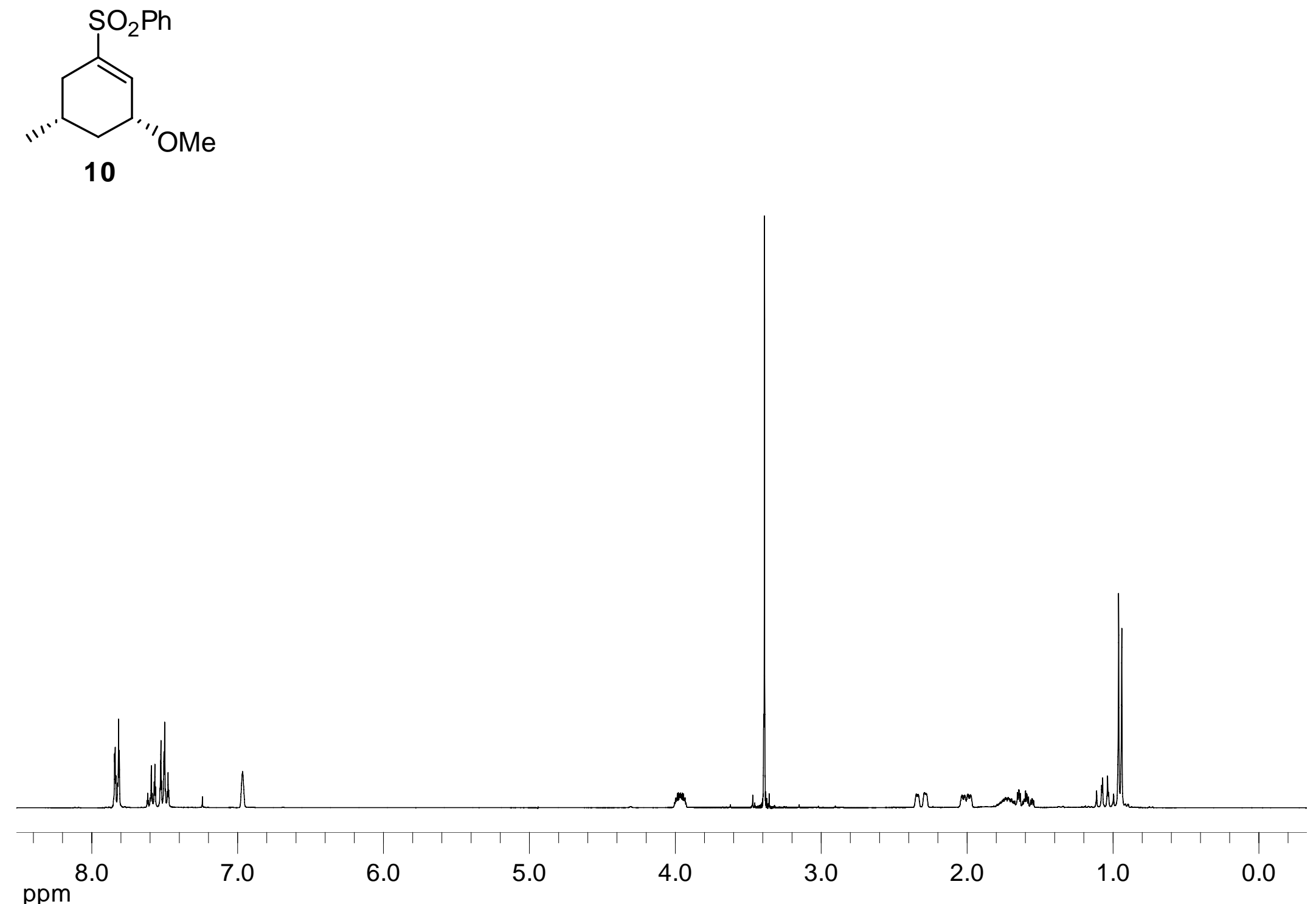

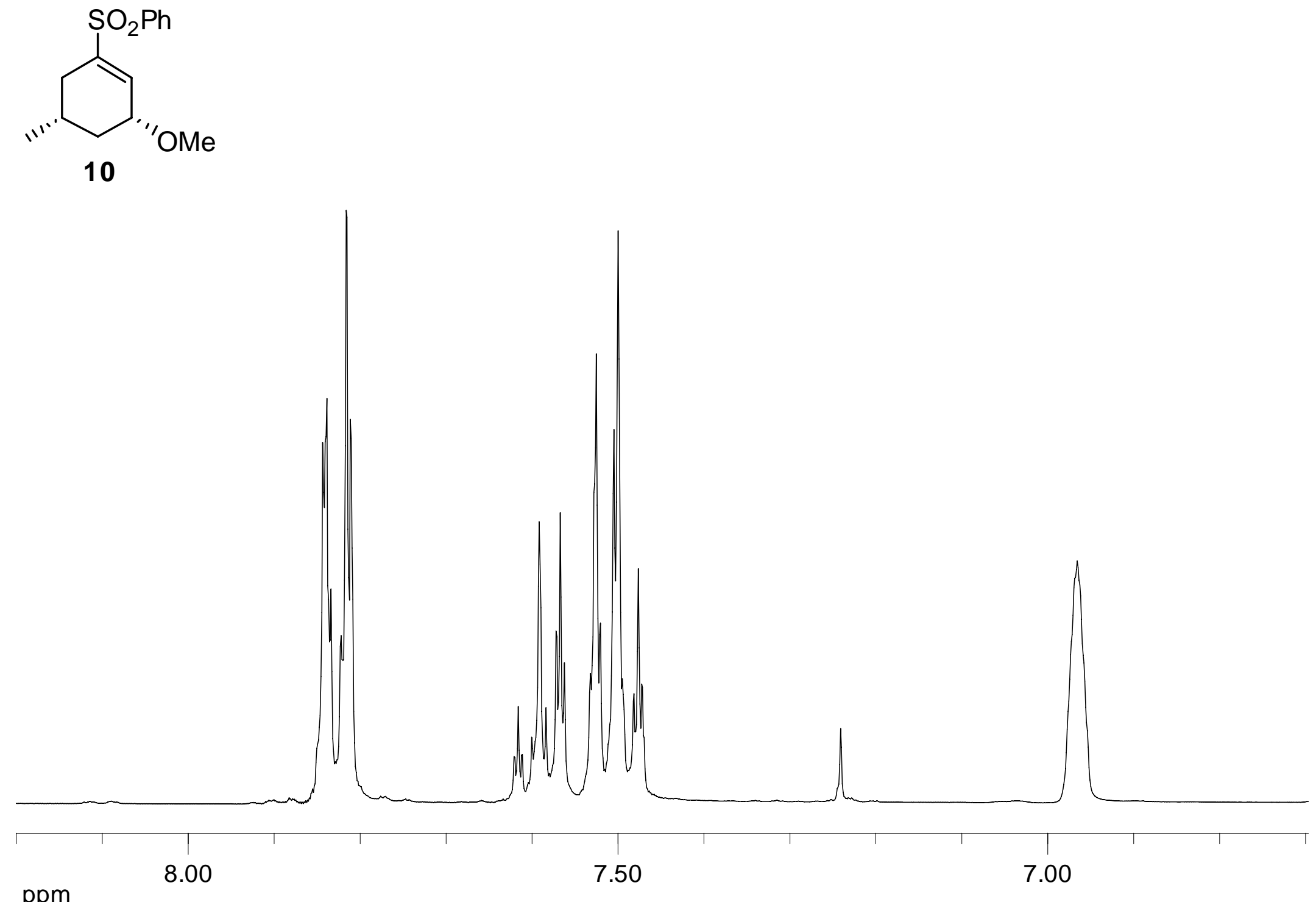

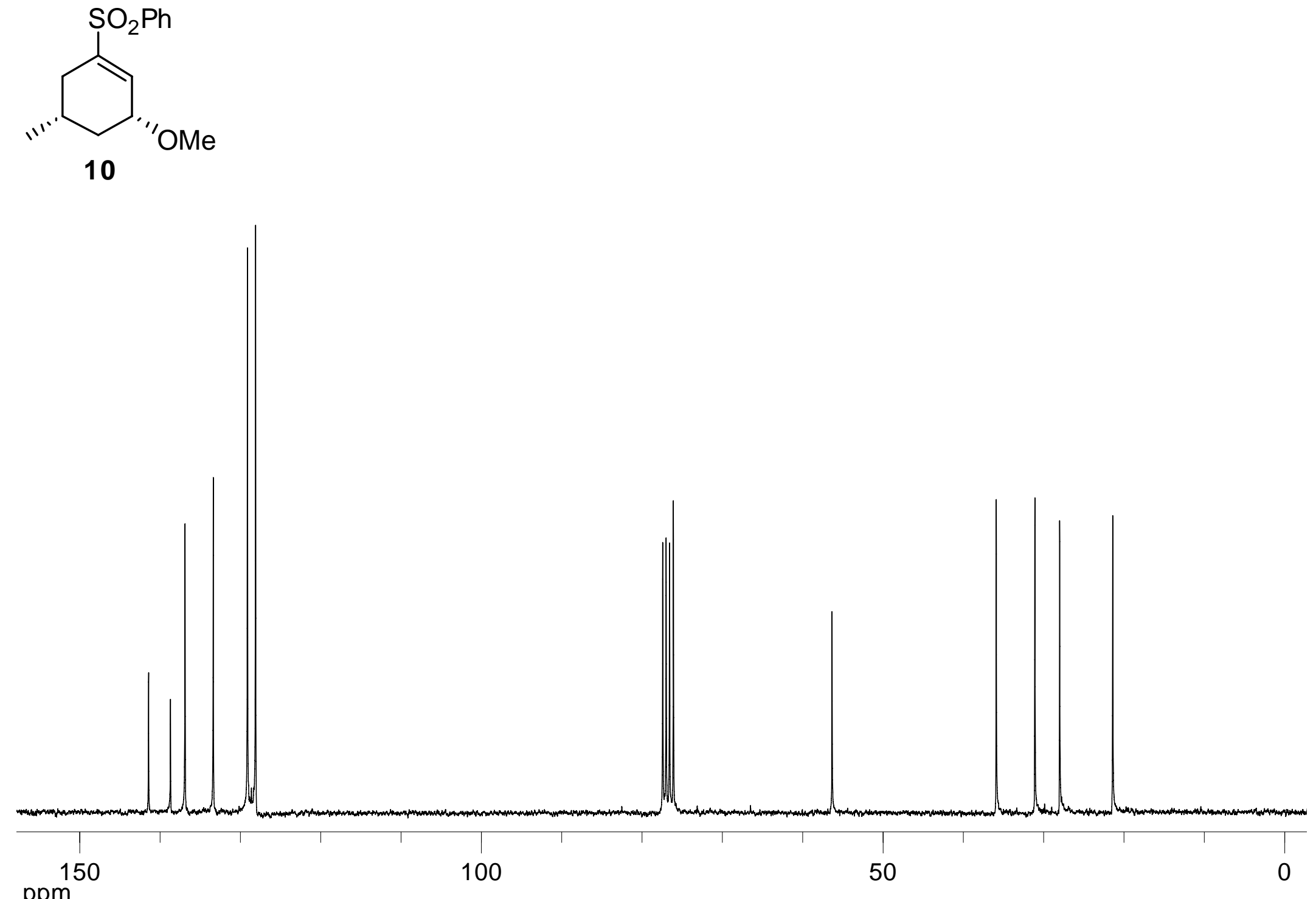


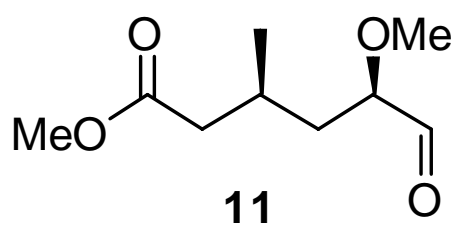

crude ${ }^{1} \mathrm{H}$ NMR containing residual DMSO

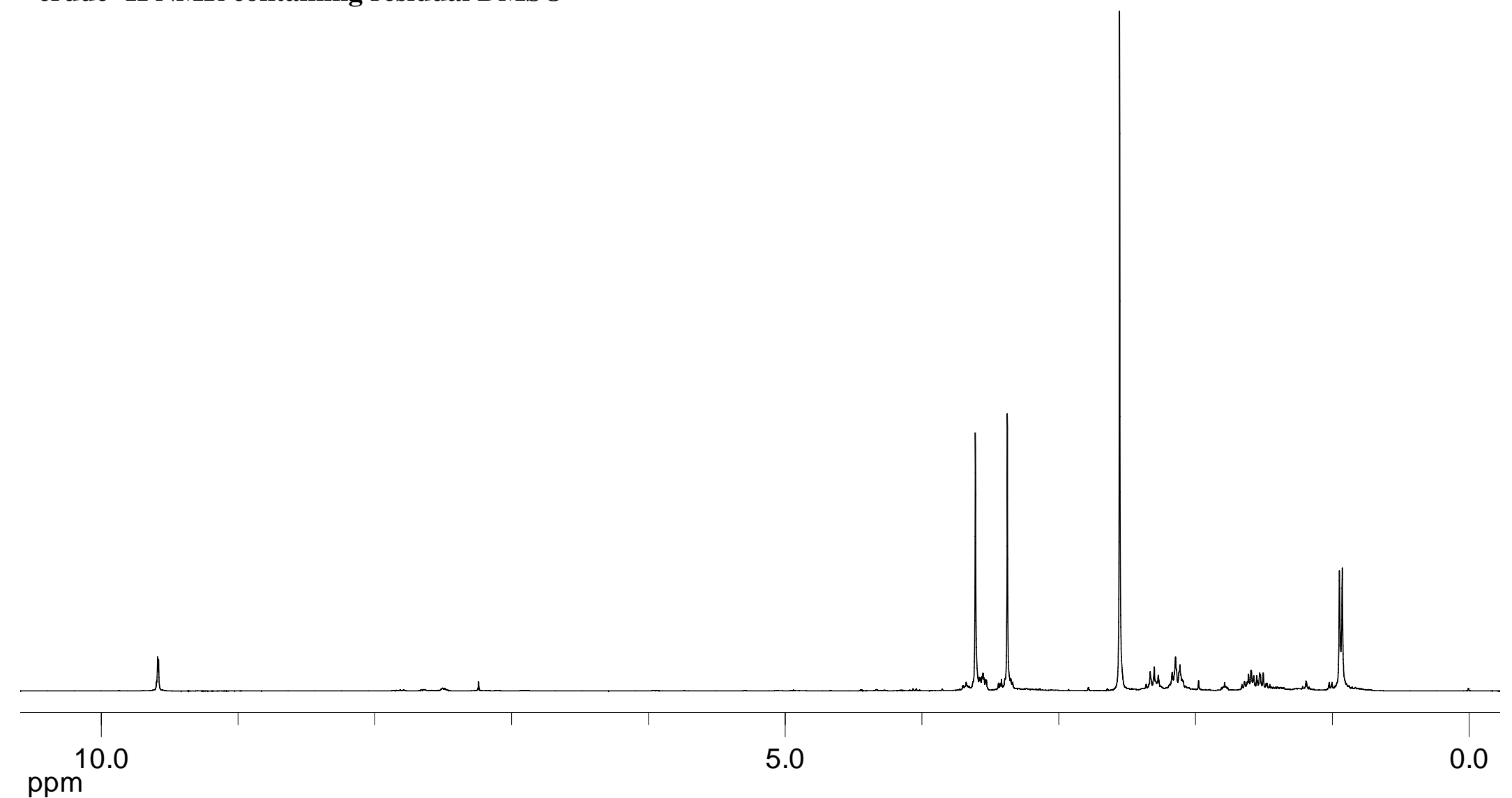




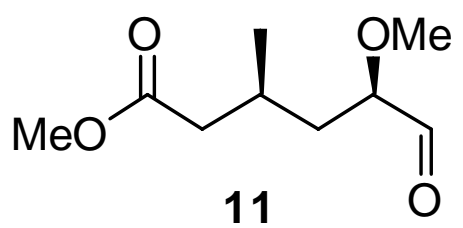

crude ${ }^{1}$ H NMR containing residual DMSO

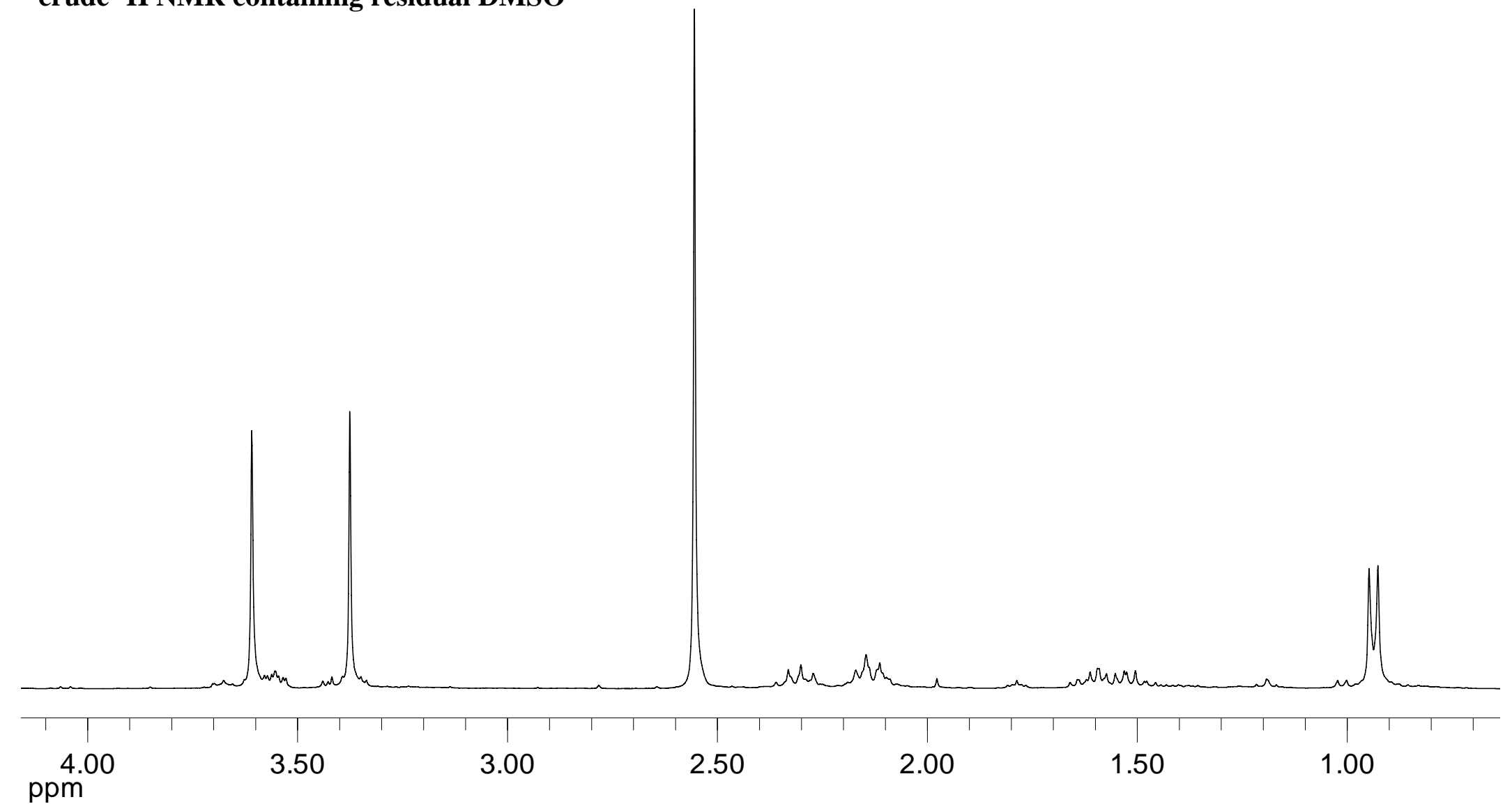



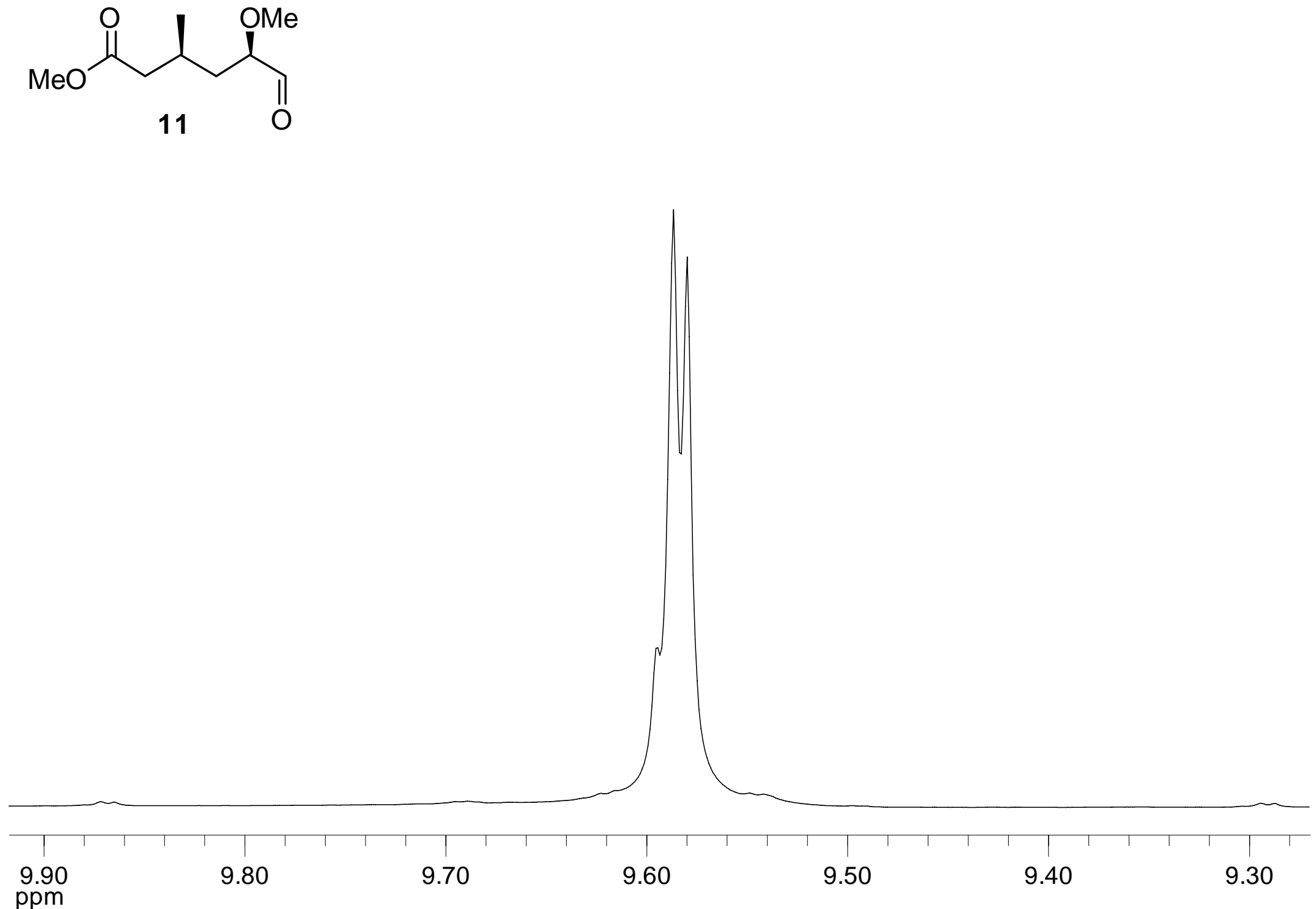

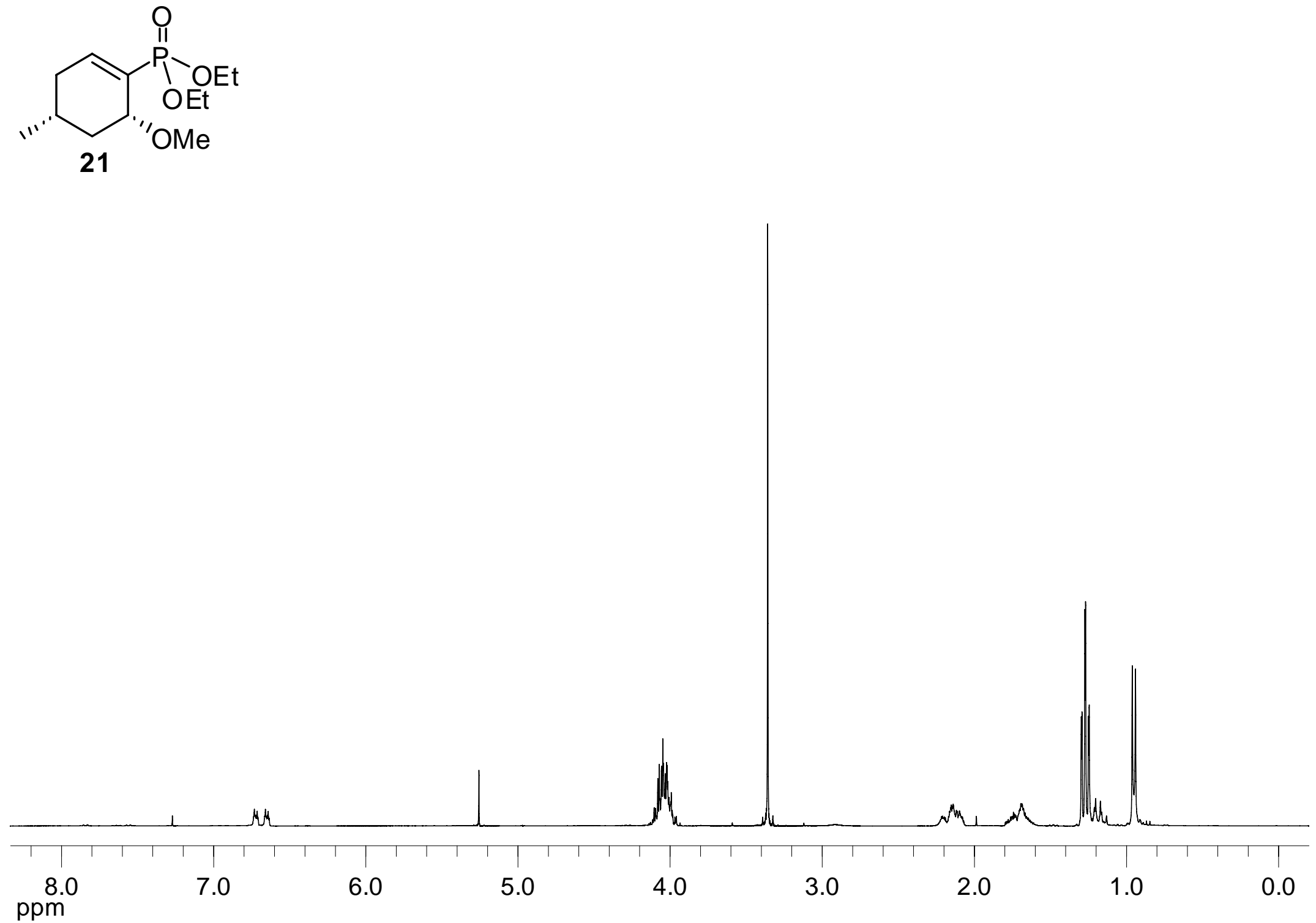

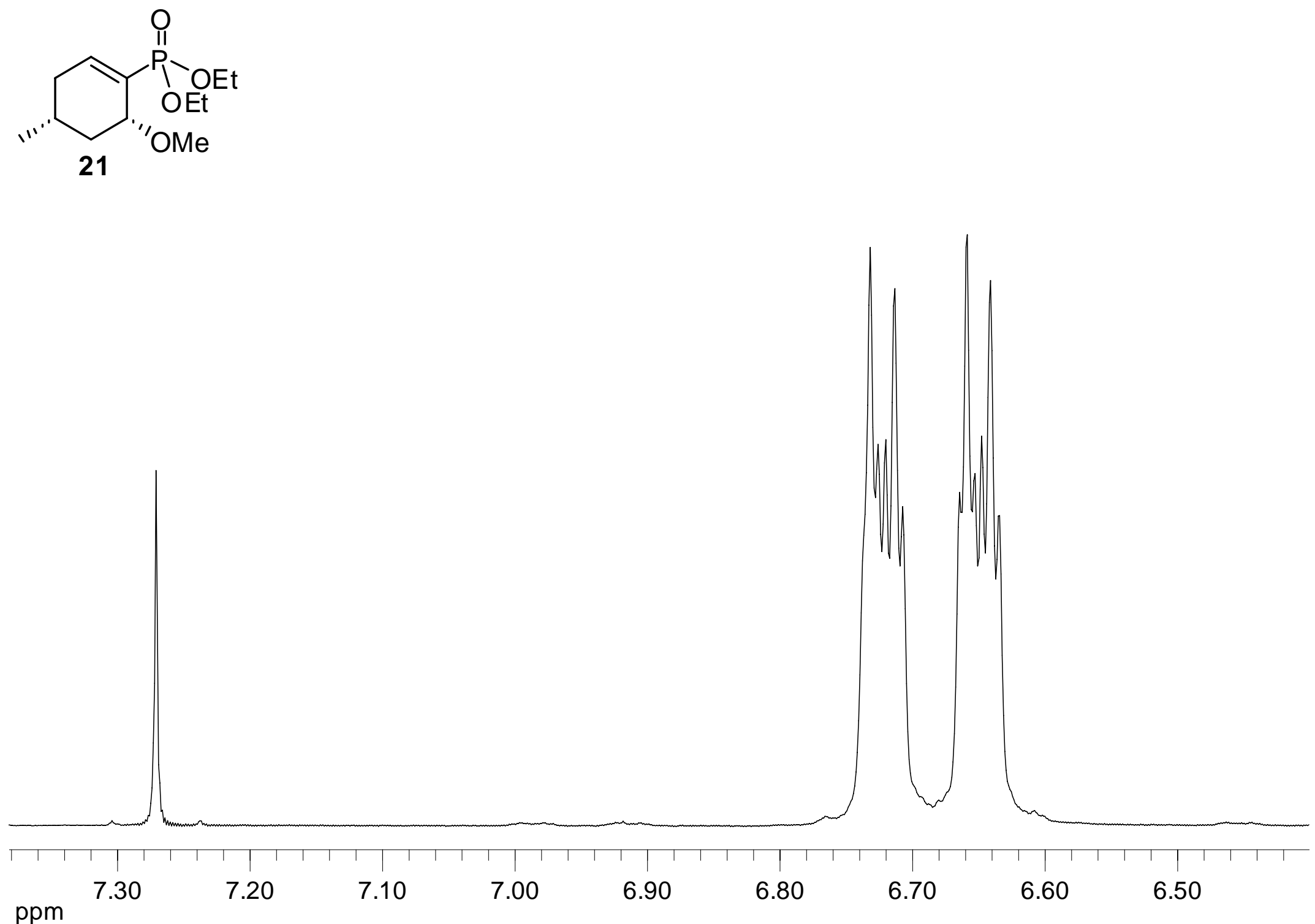

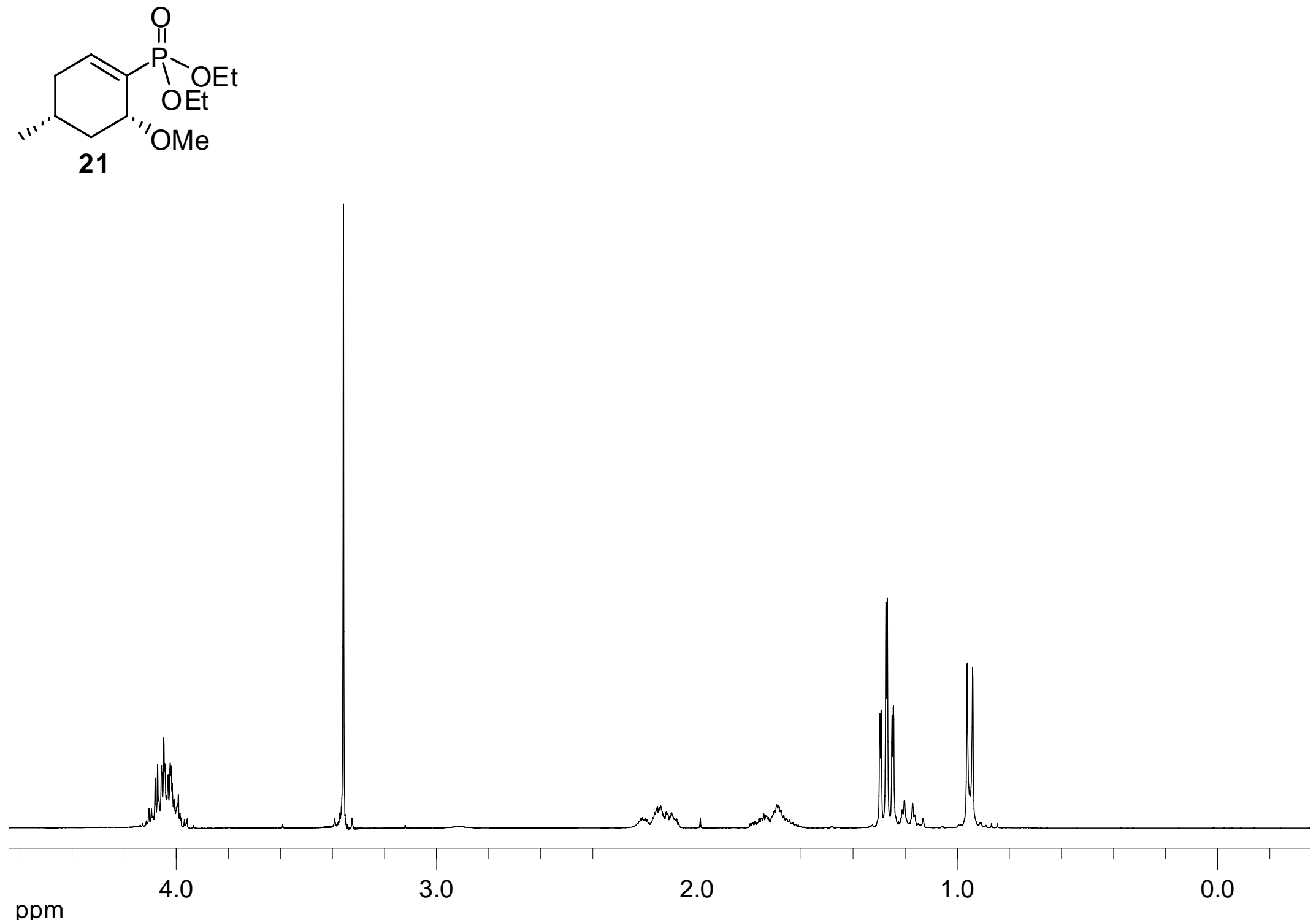

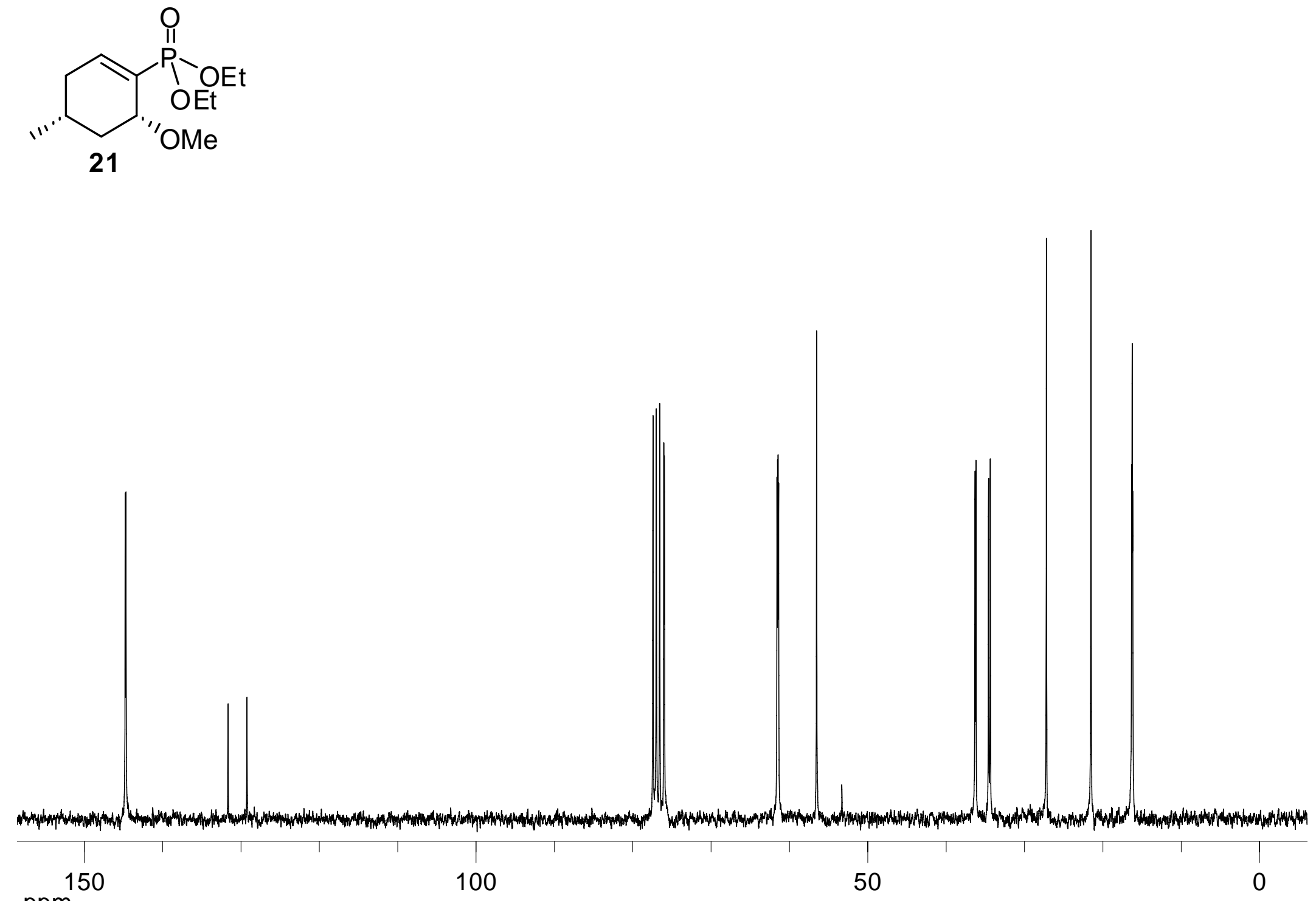
ppm 


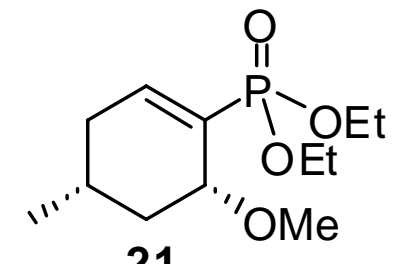

21

.

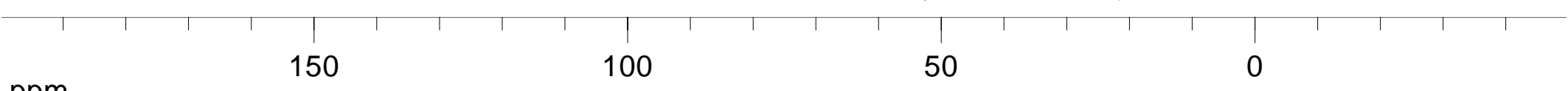

ppm

150

100

50 

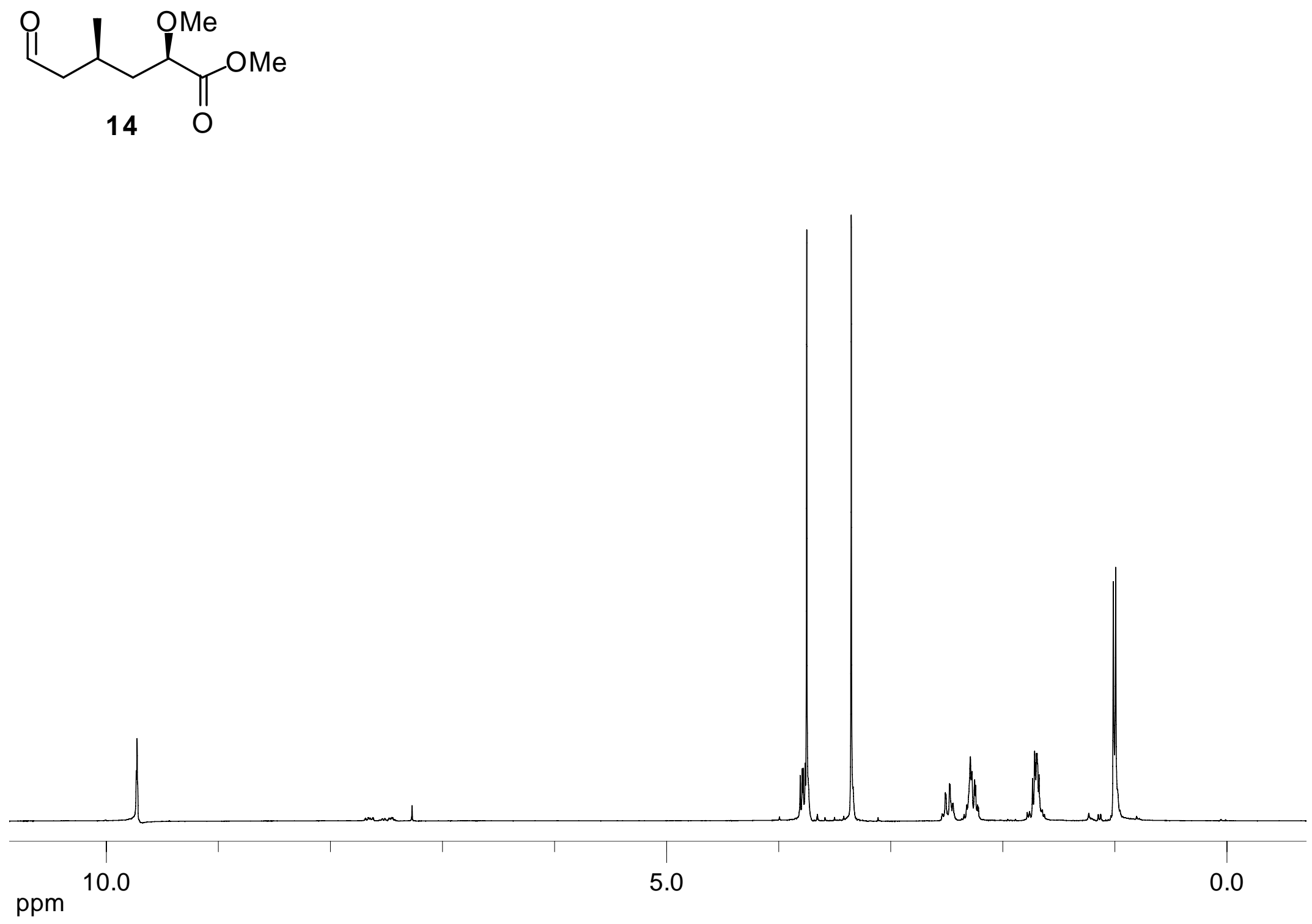

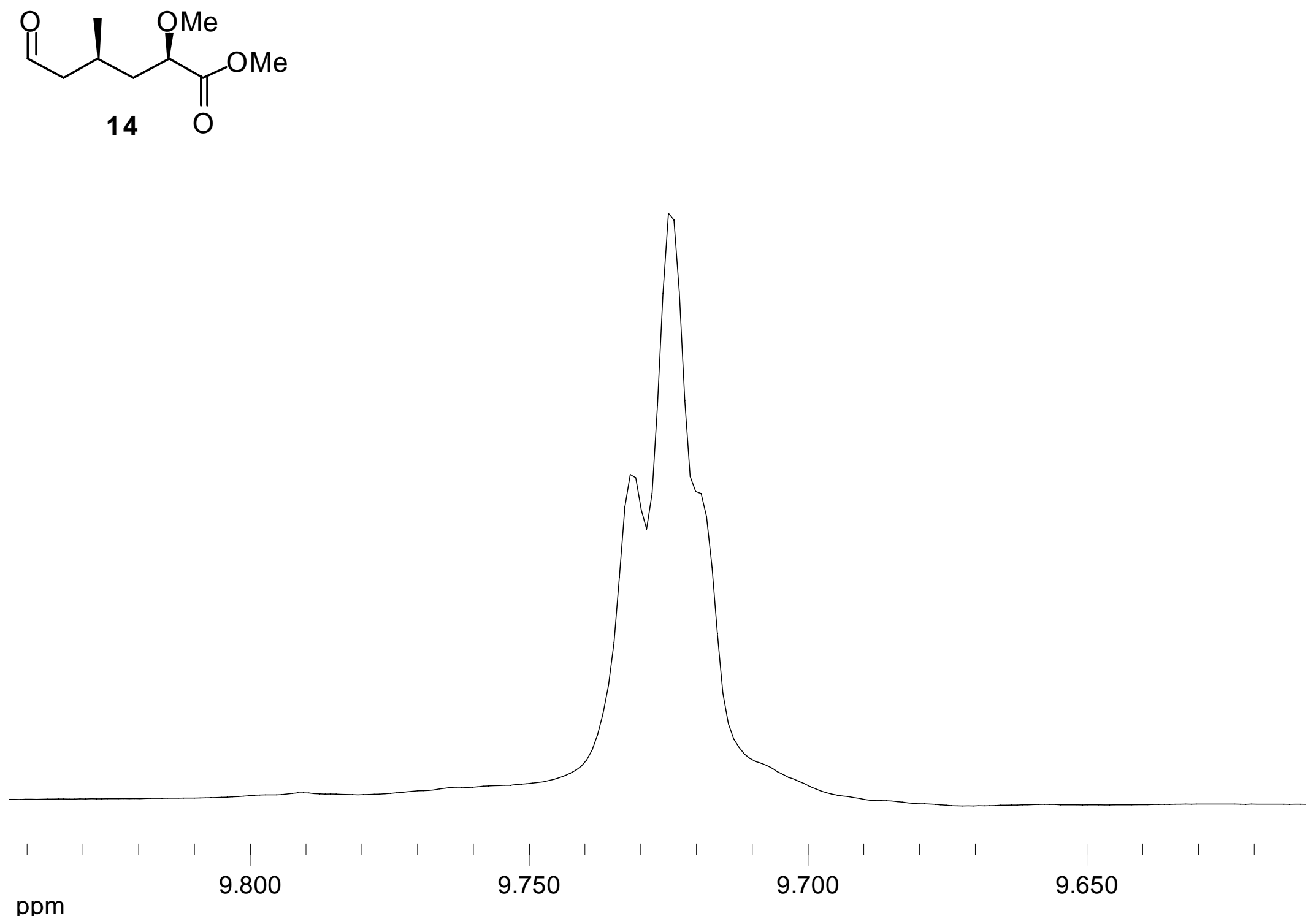

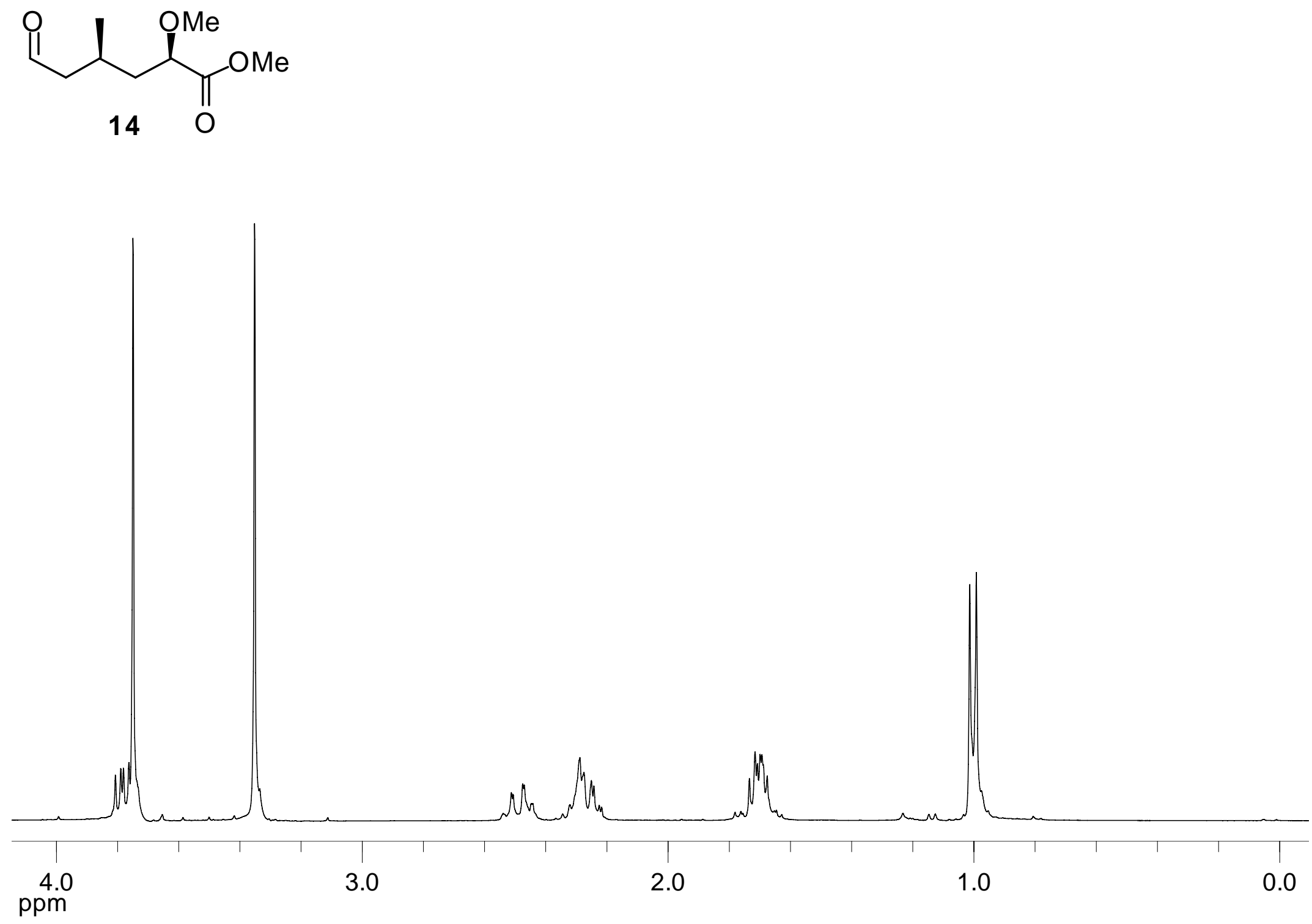

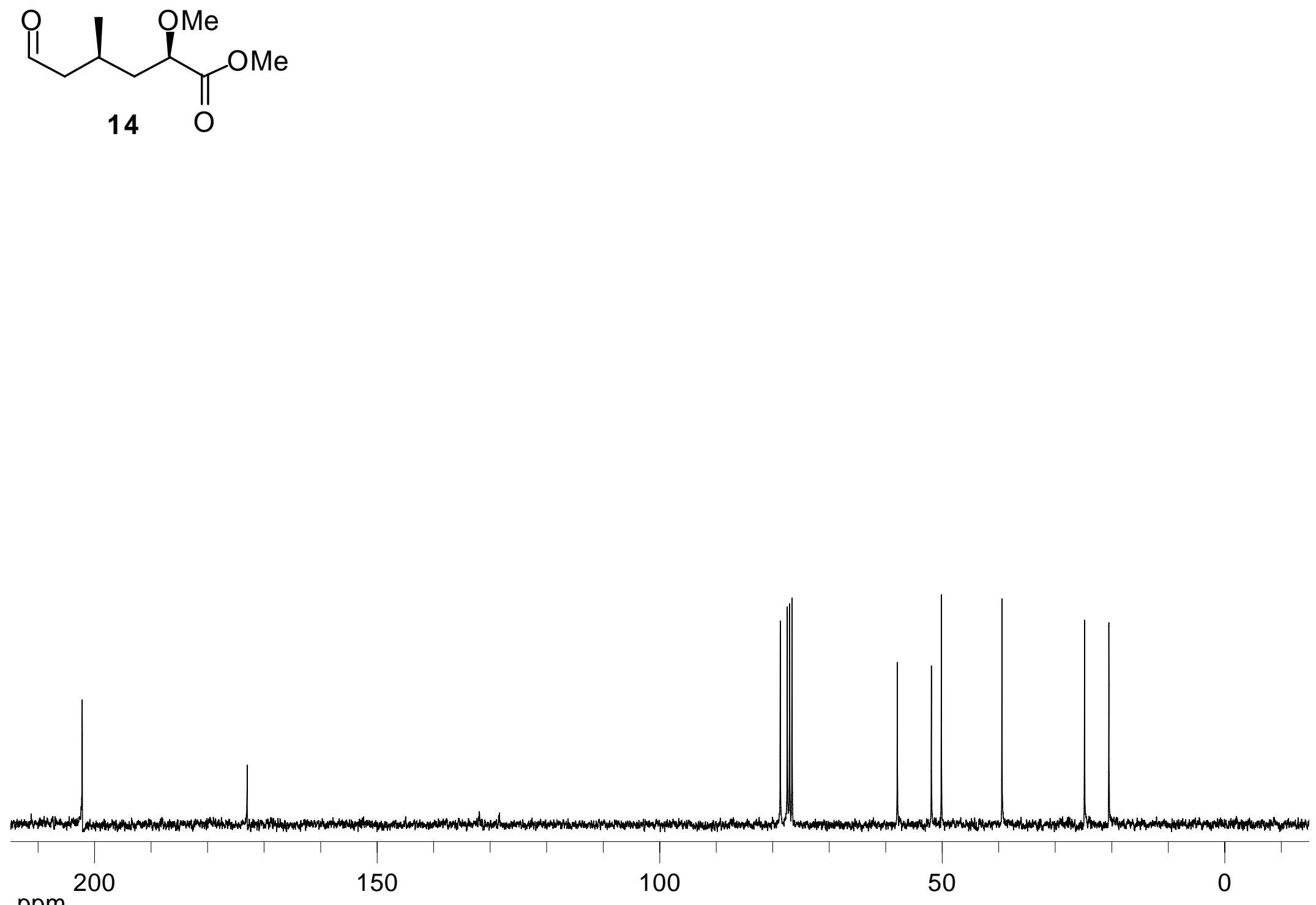
ppm 

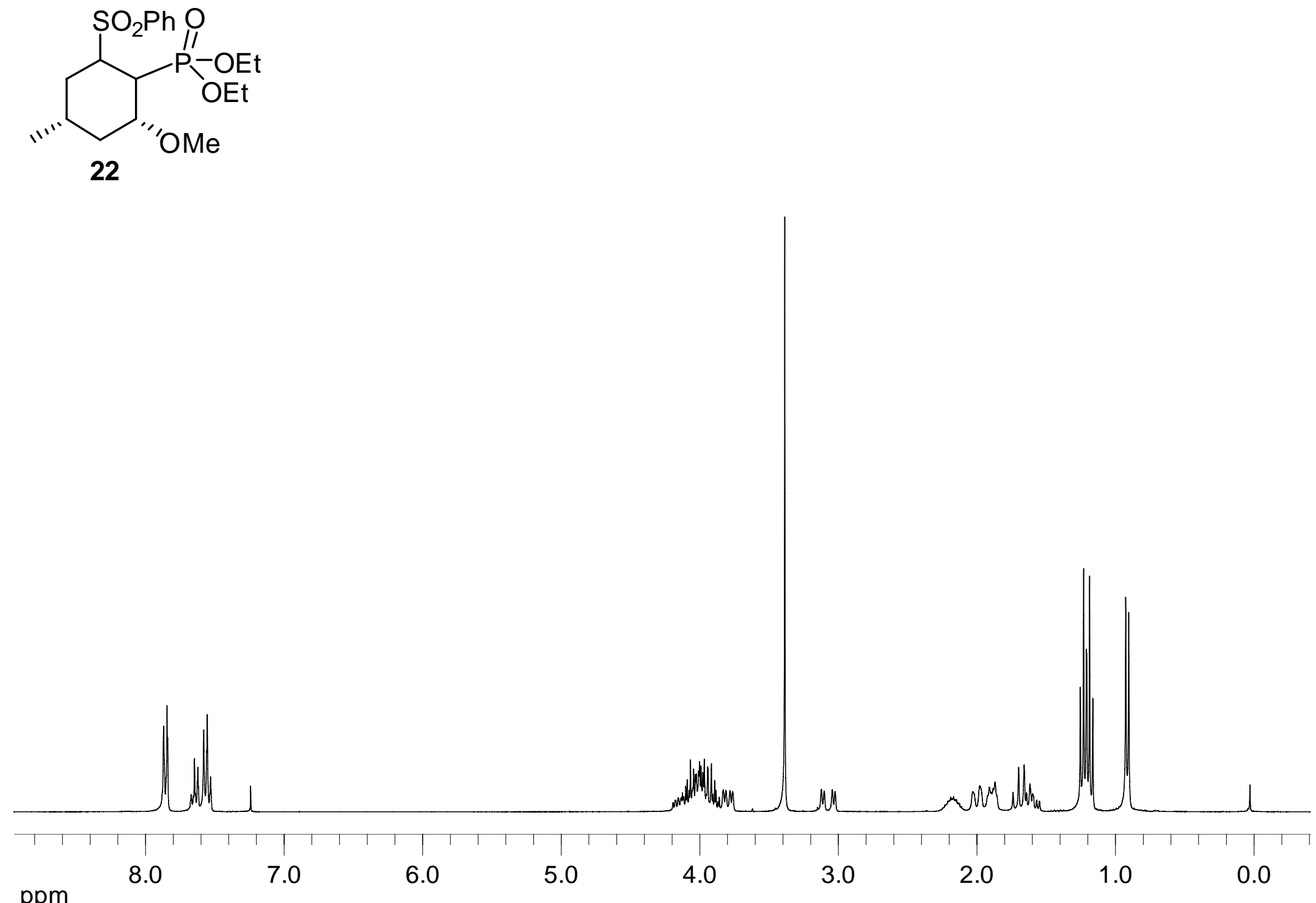

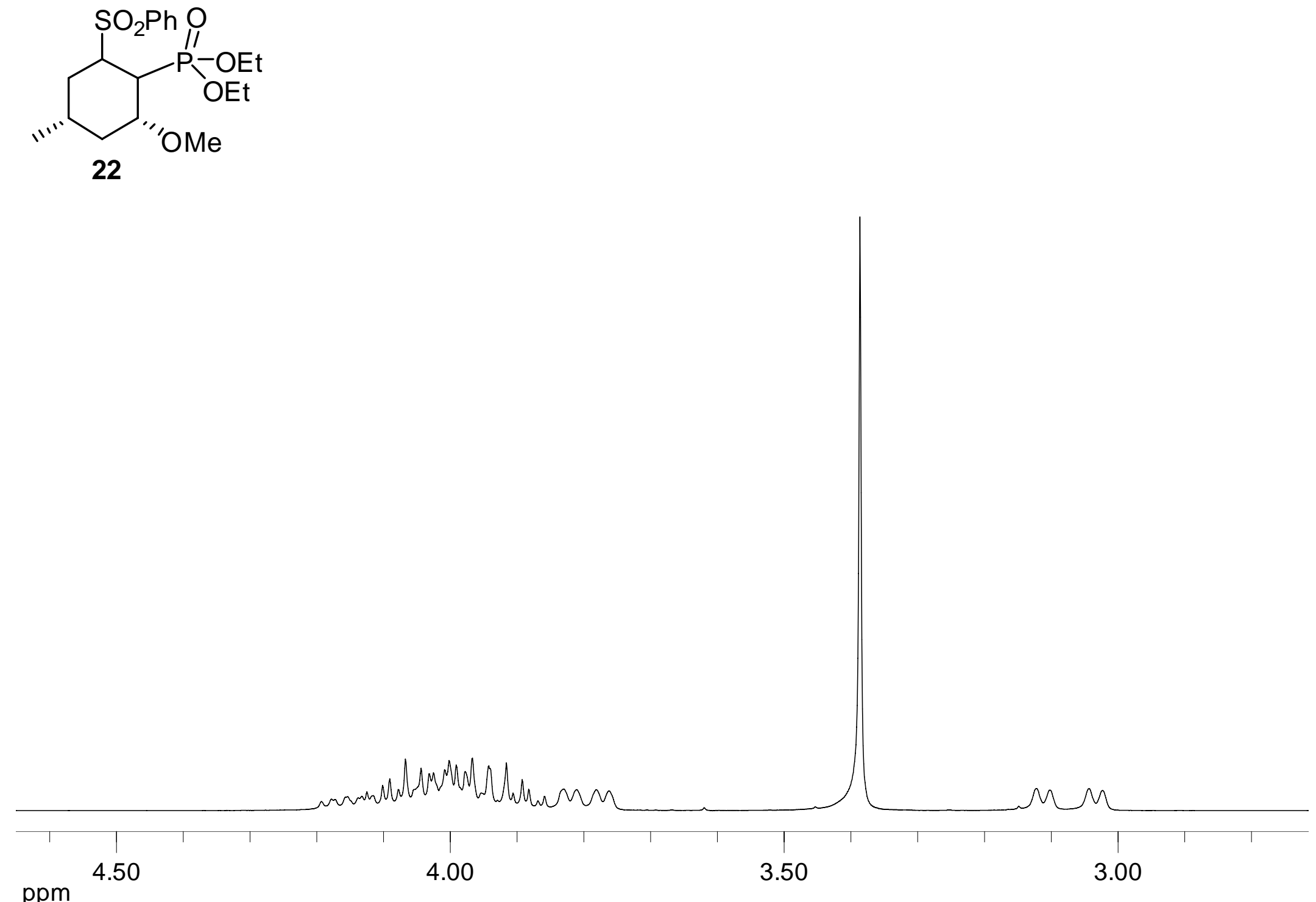

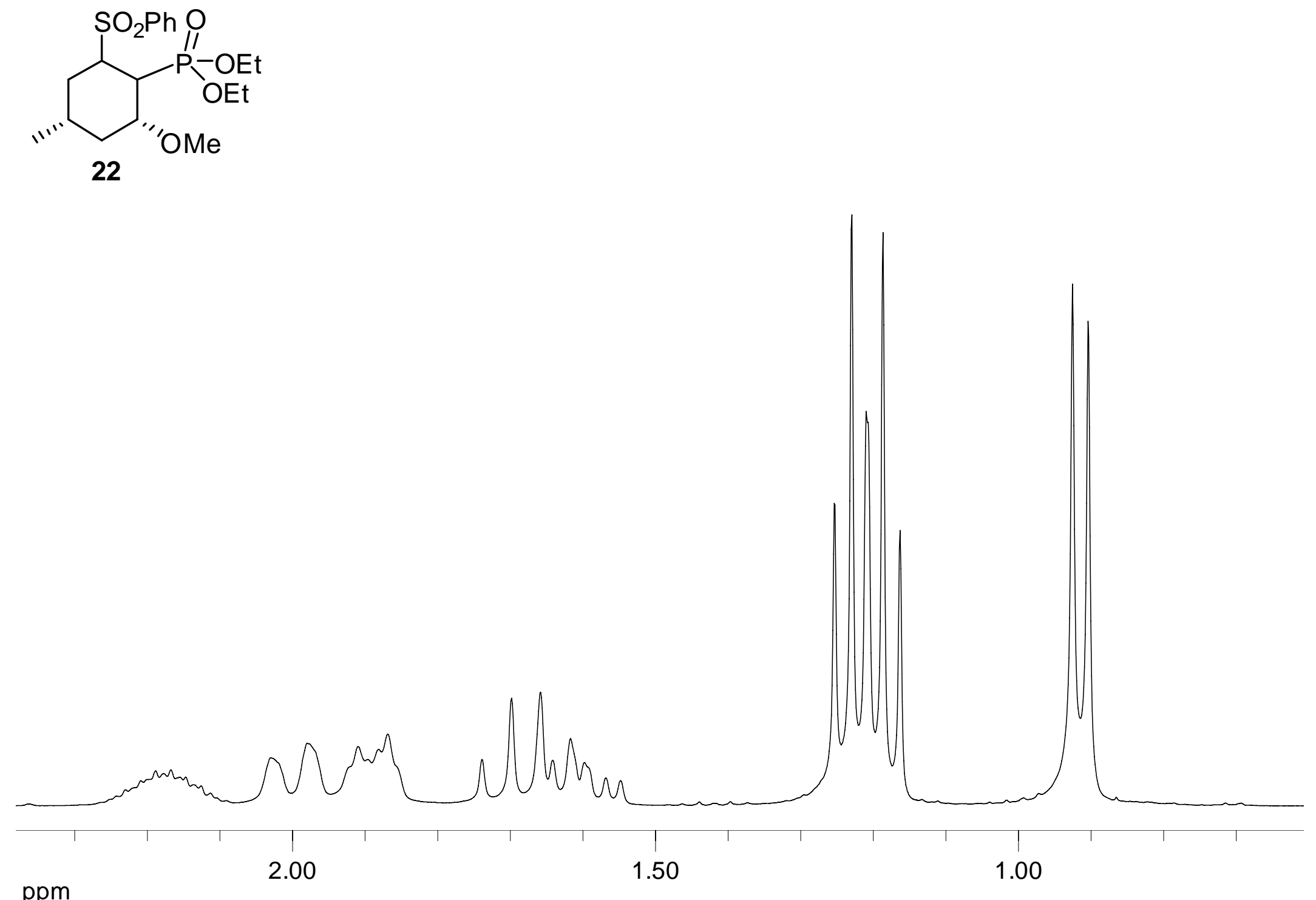

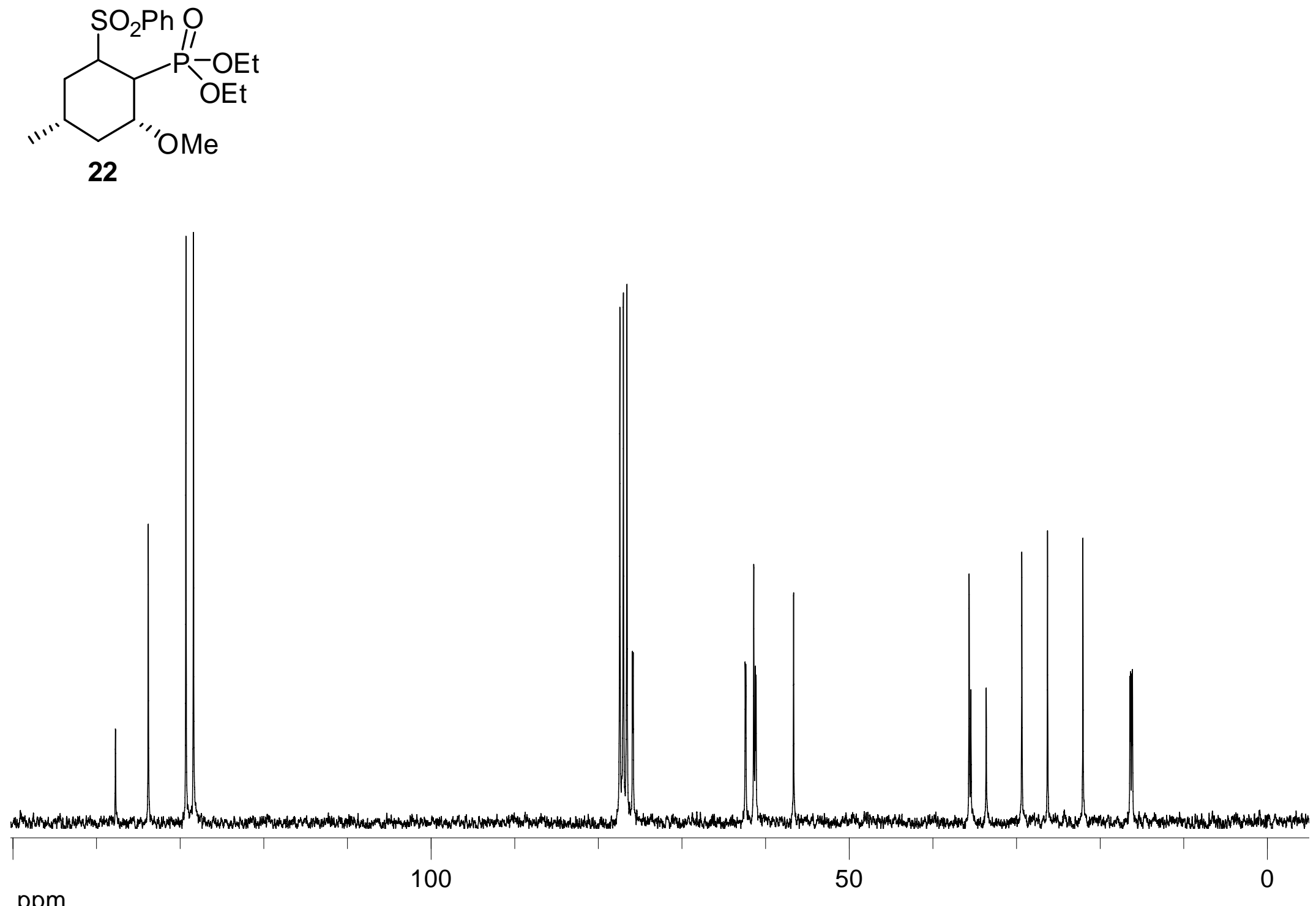

ppm 

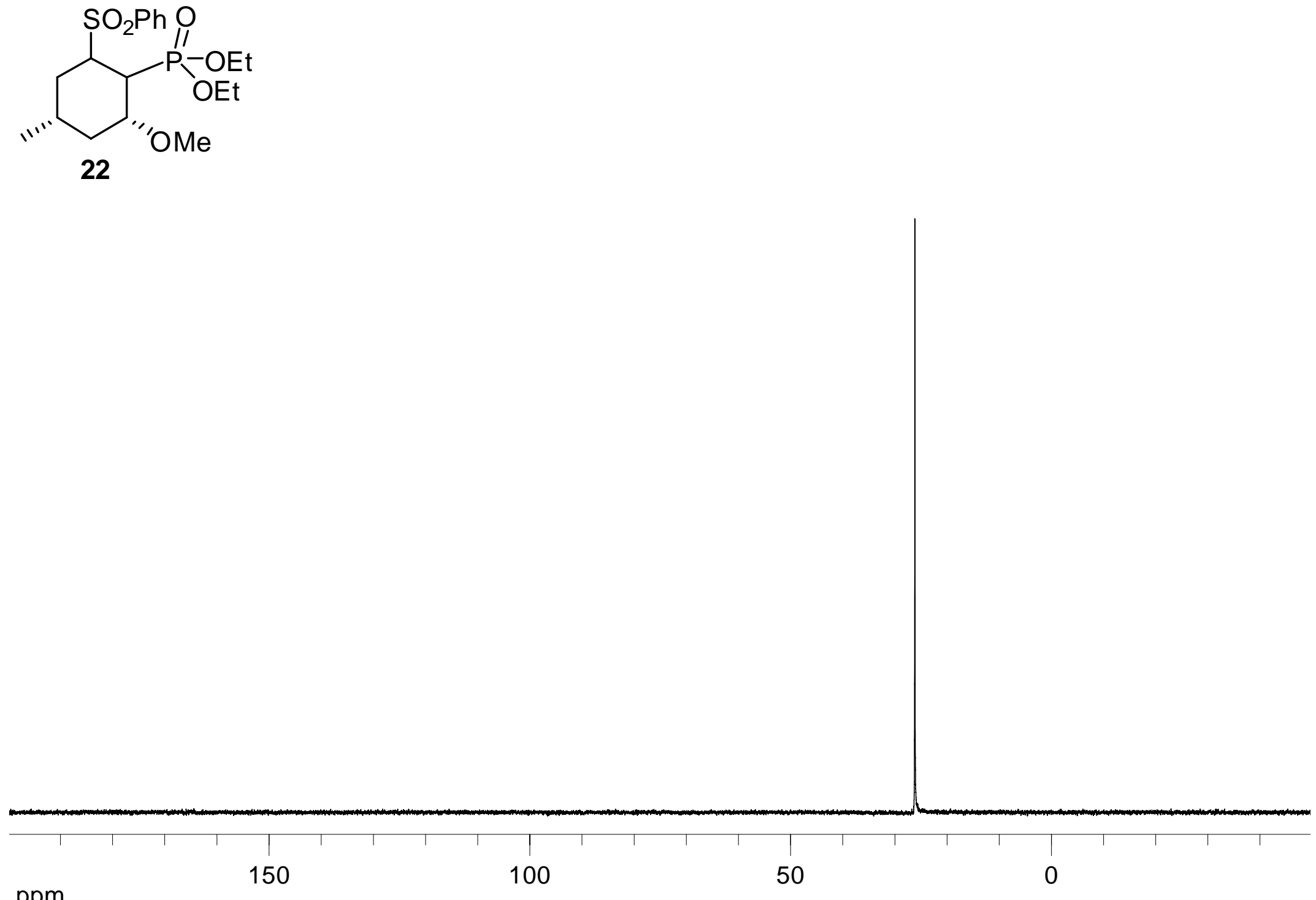

ppm 

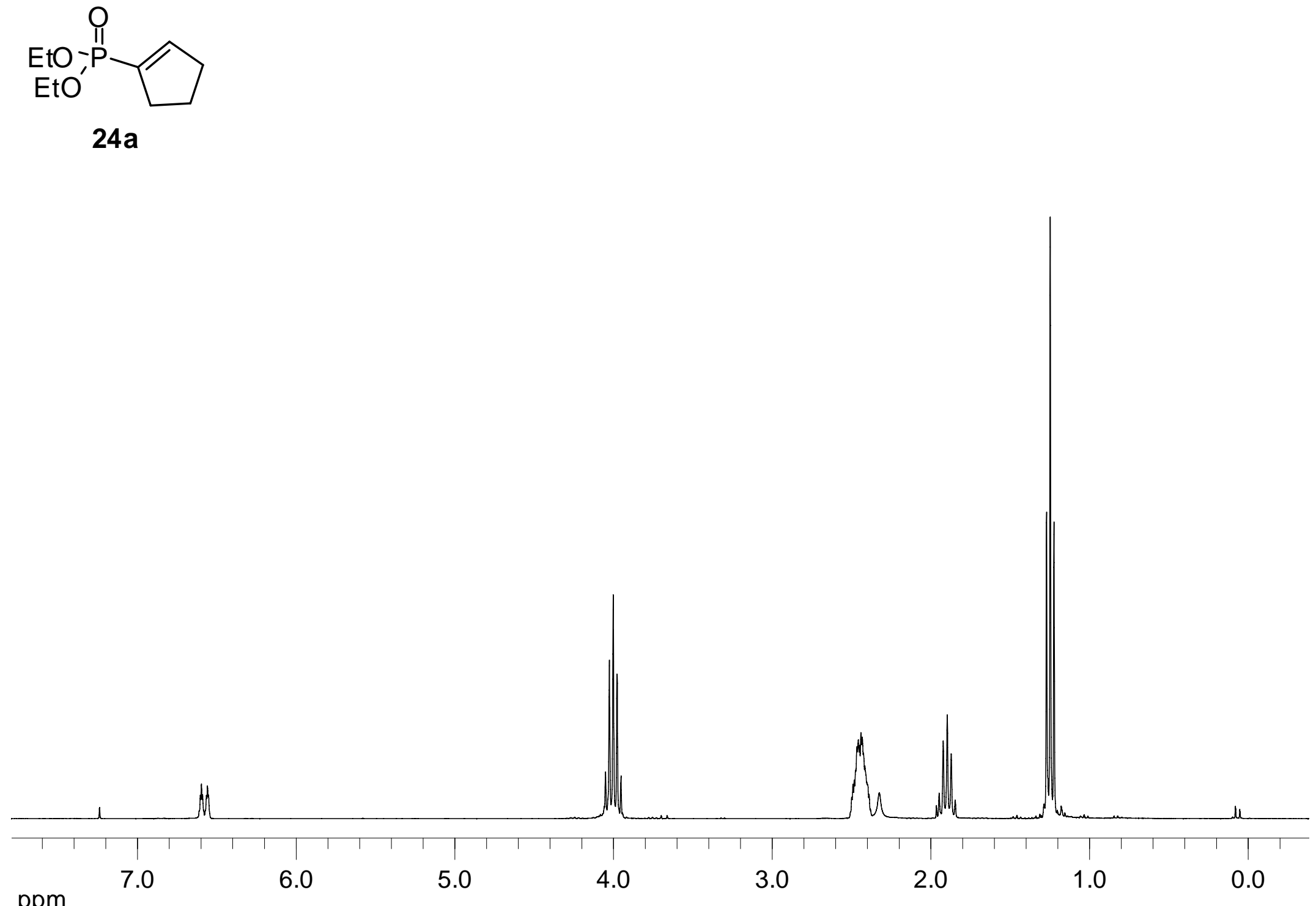

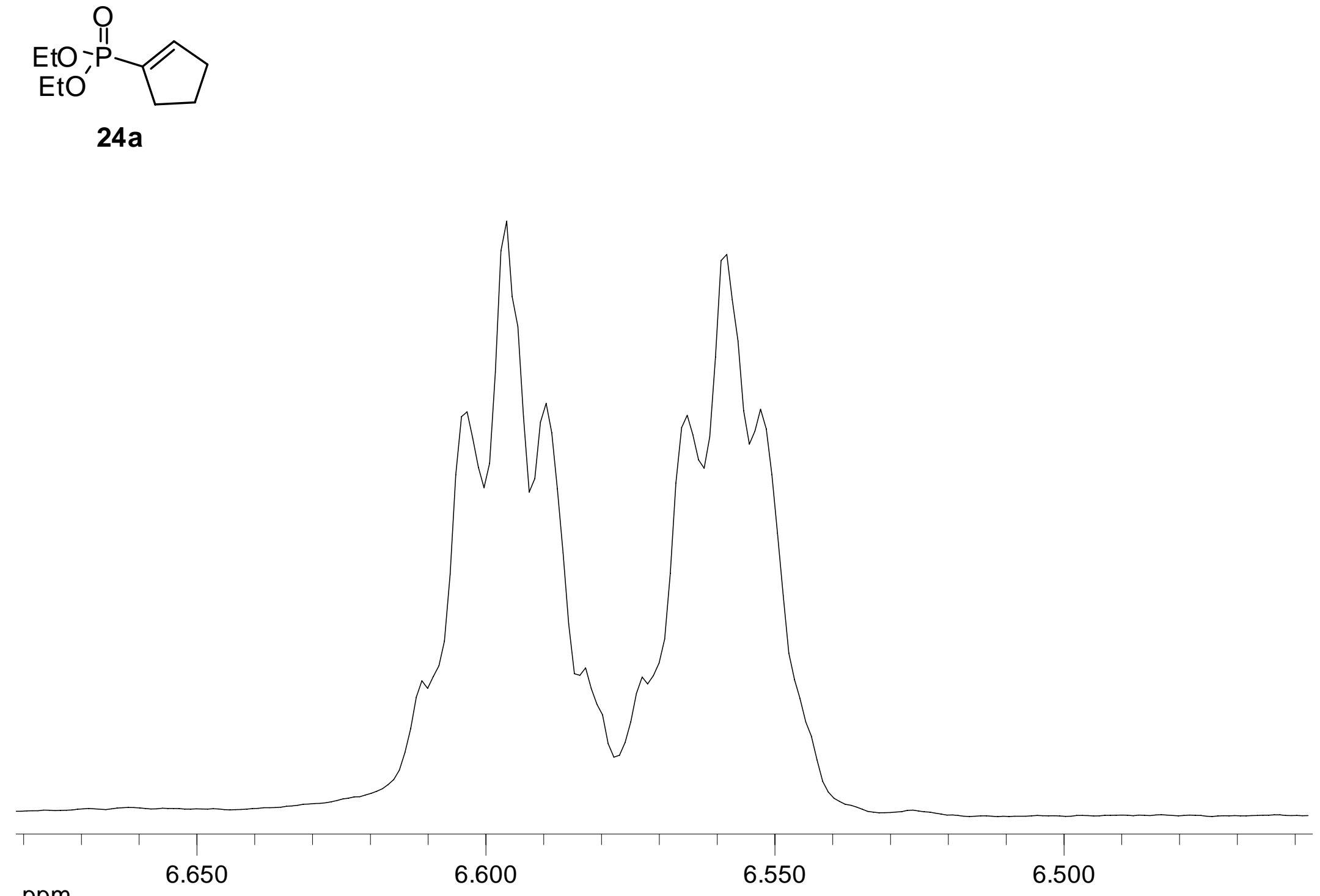

ppm 

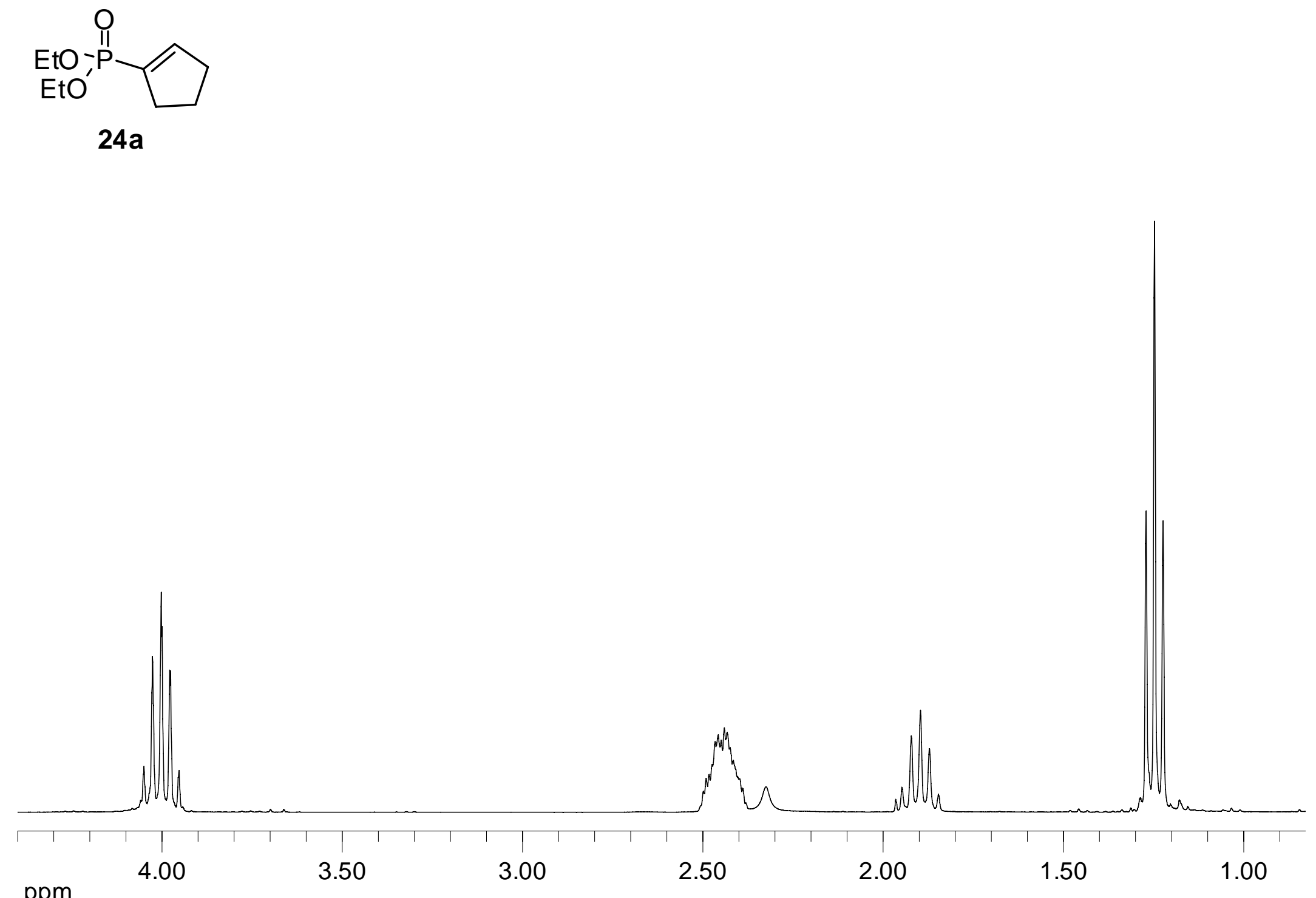


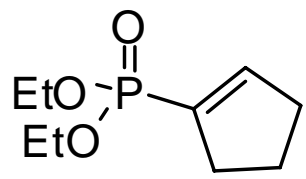

$24 a$

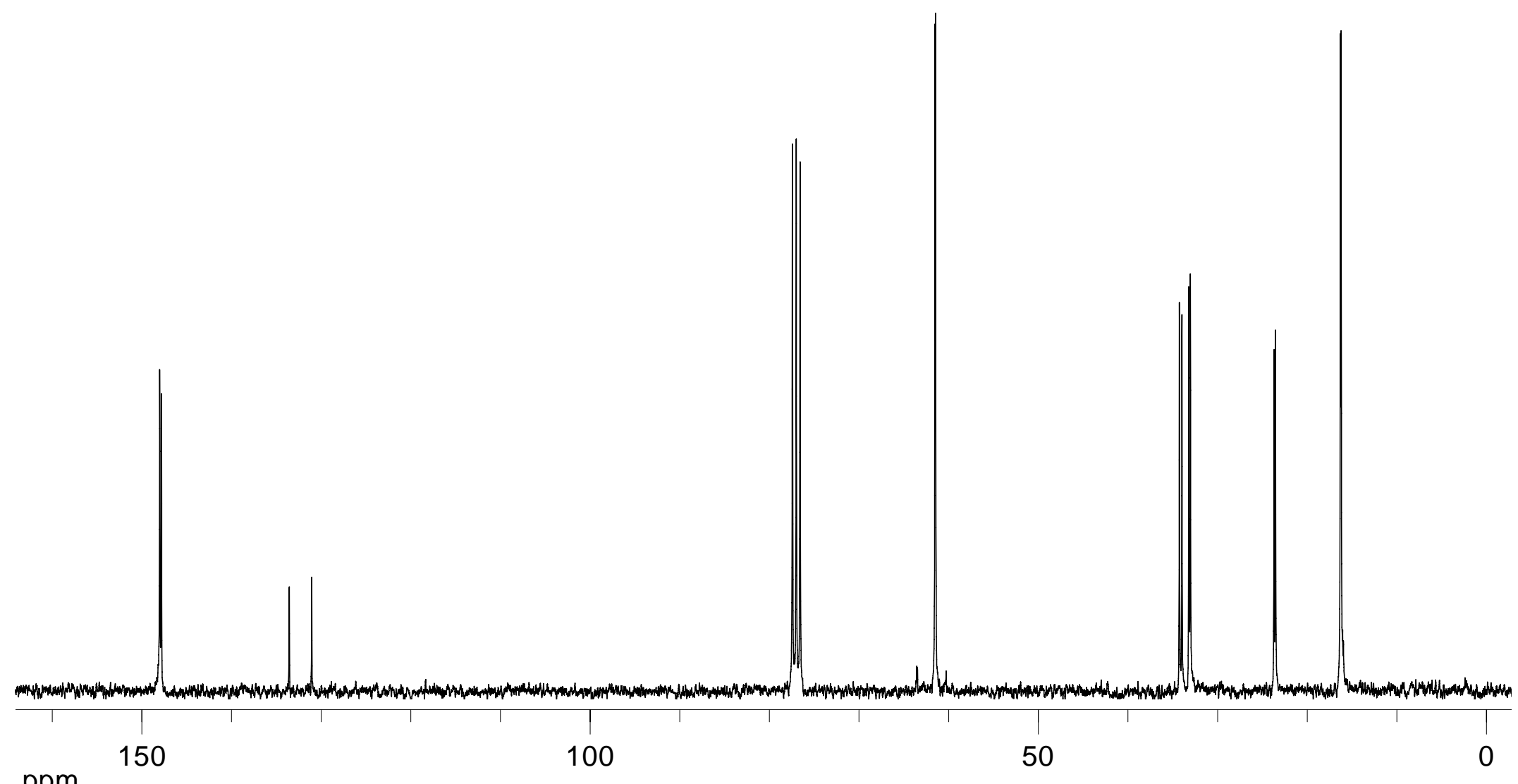



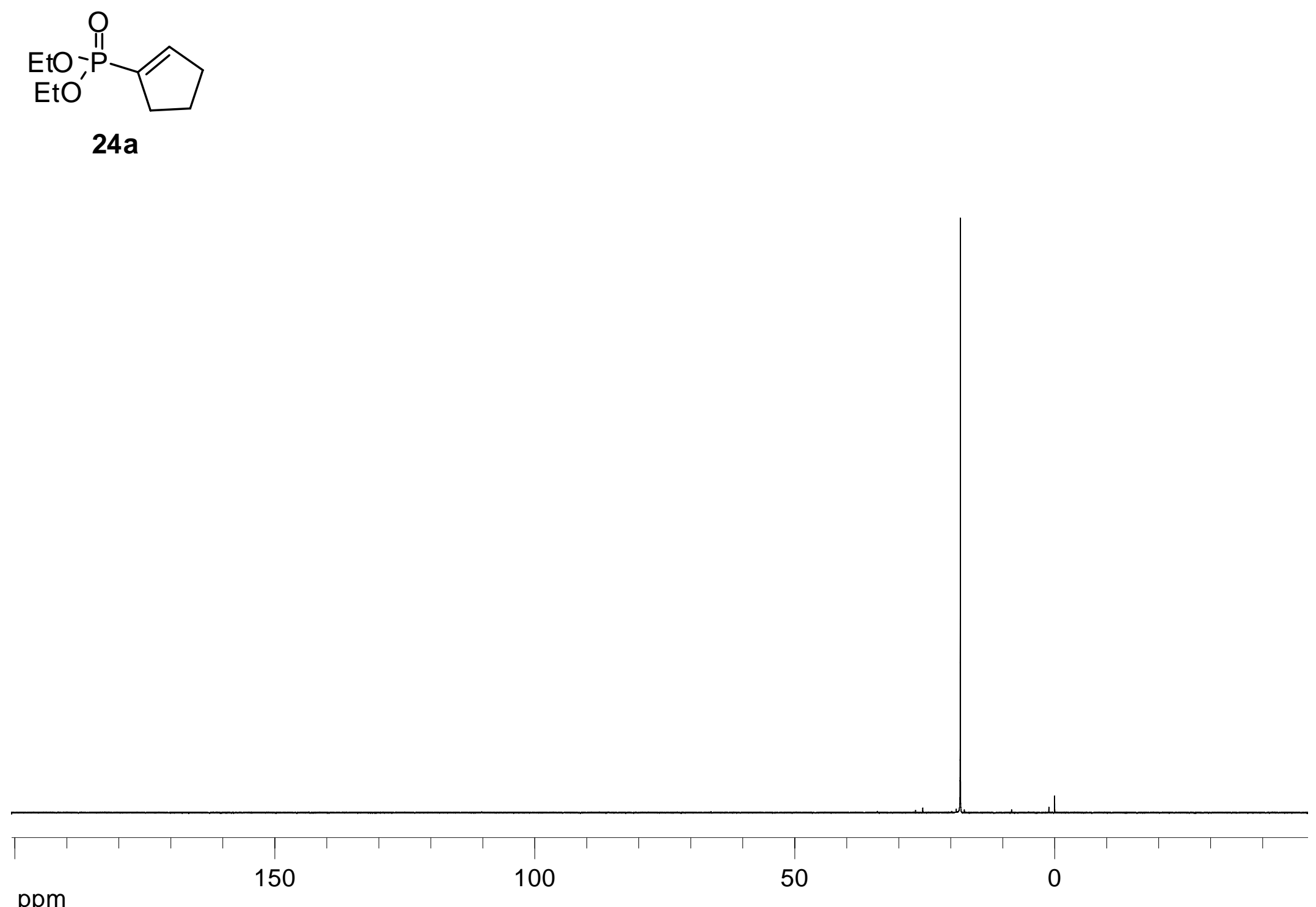


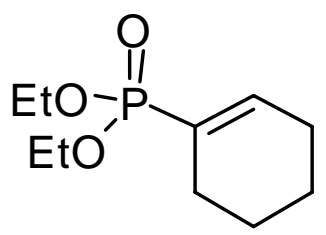

$25 a$

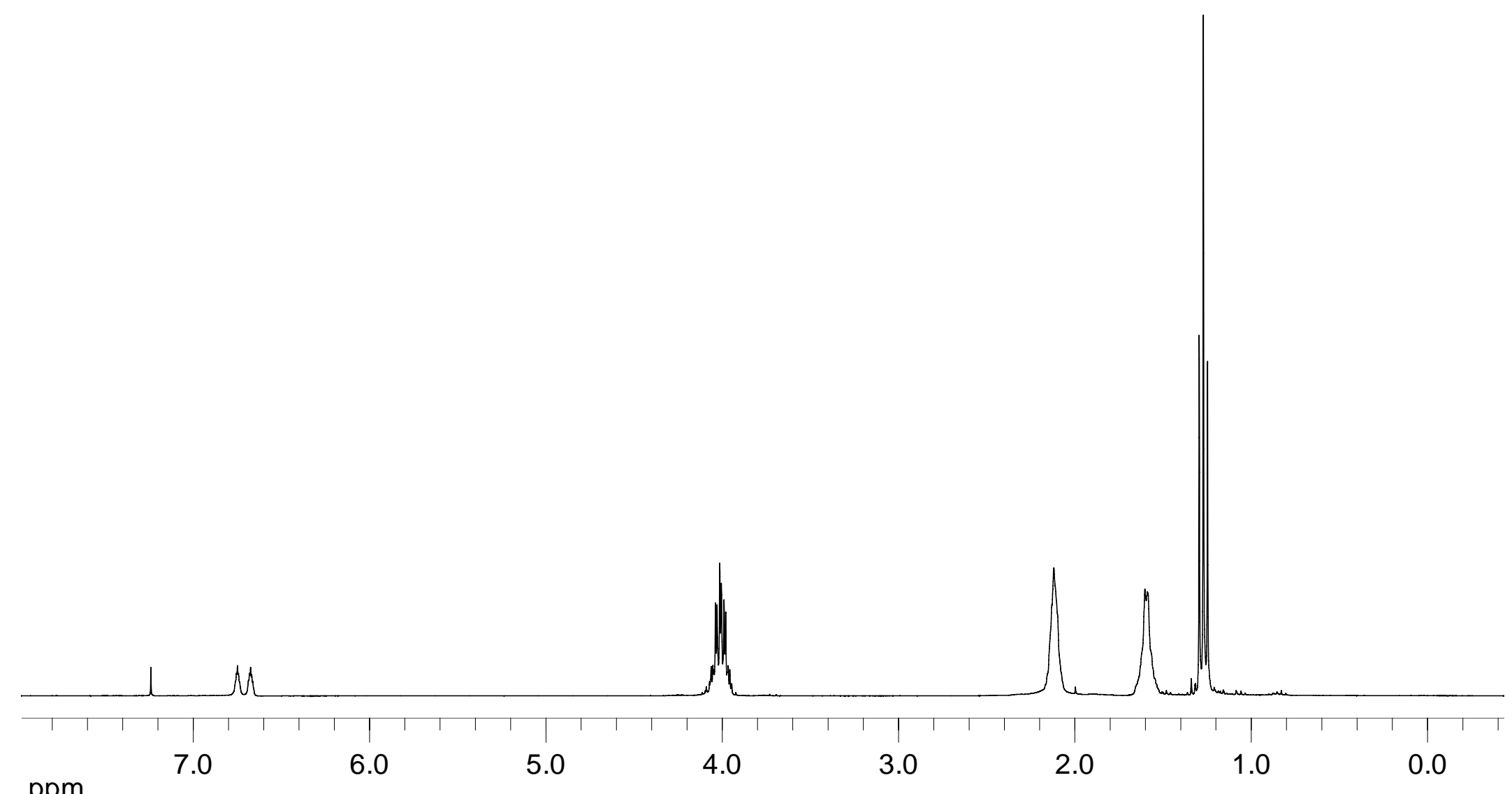




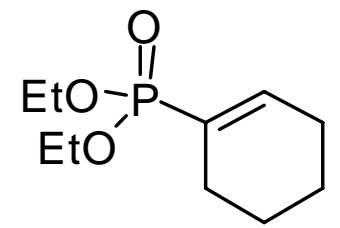

$25 a$

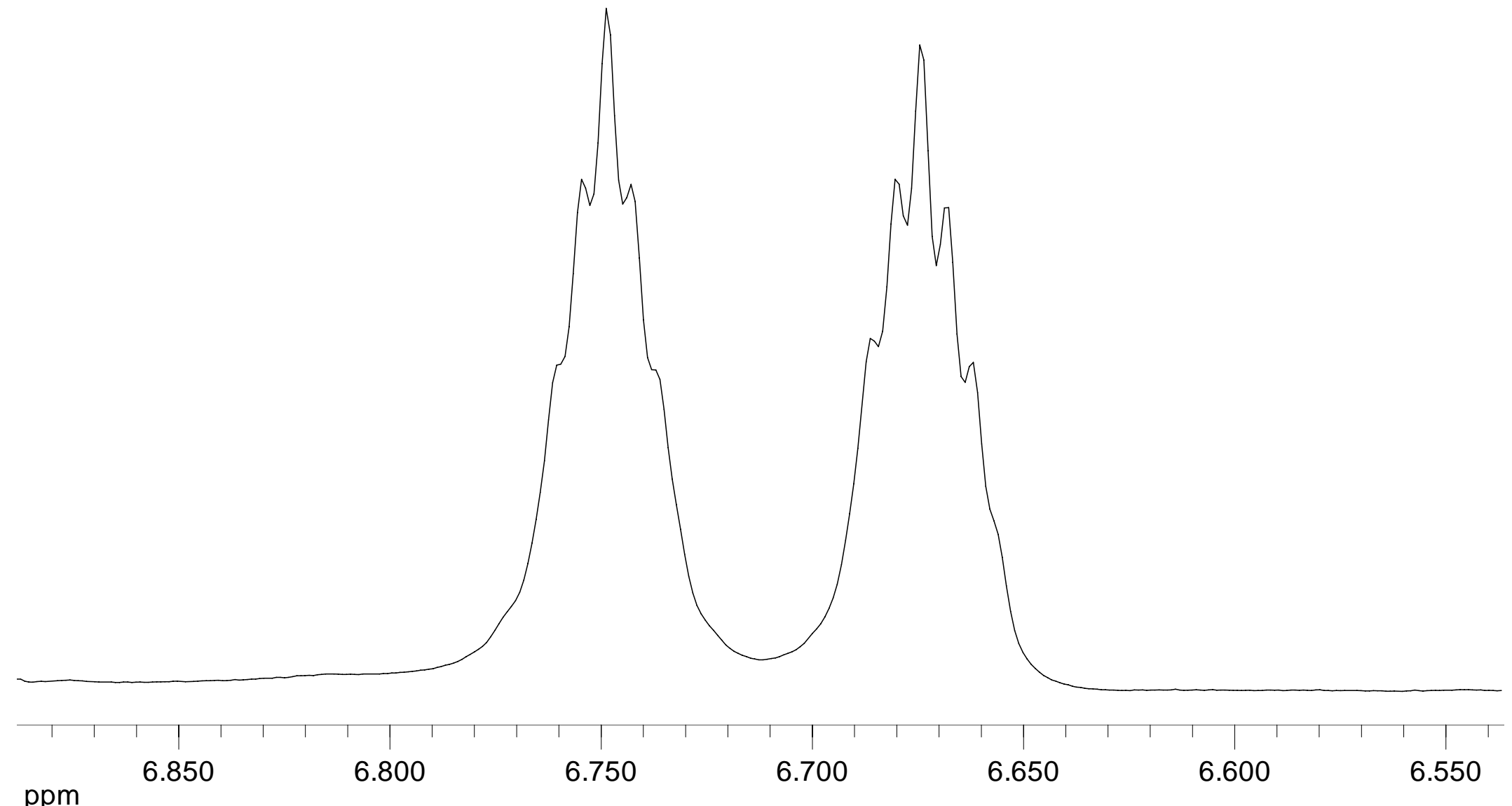




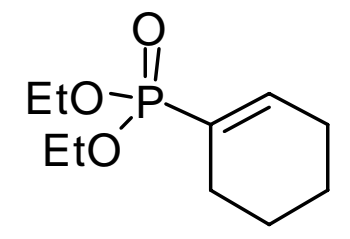

$25 a$

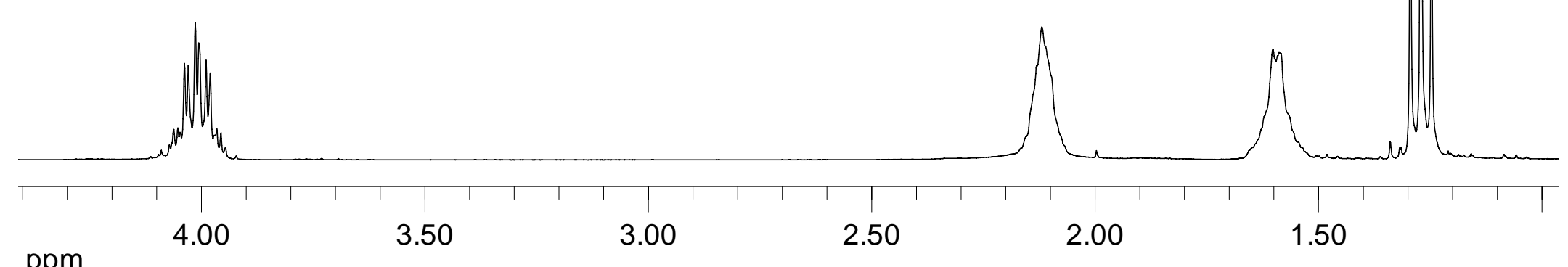

ppm 


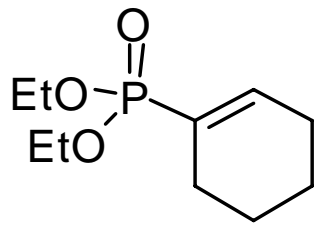

$25 a$

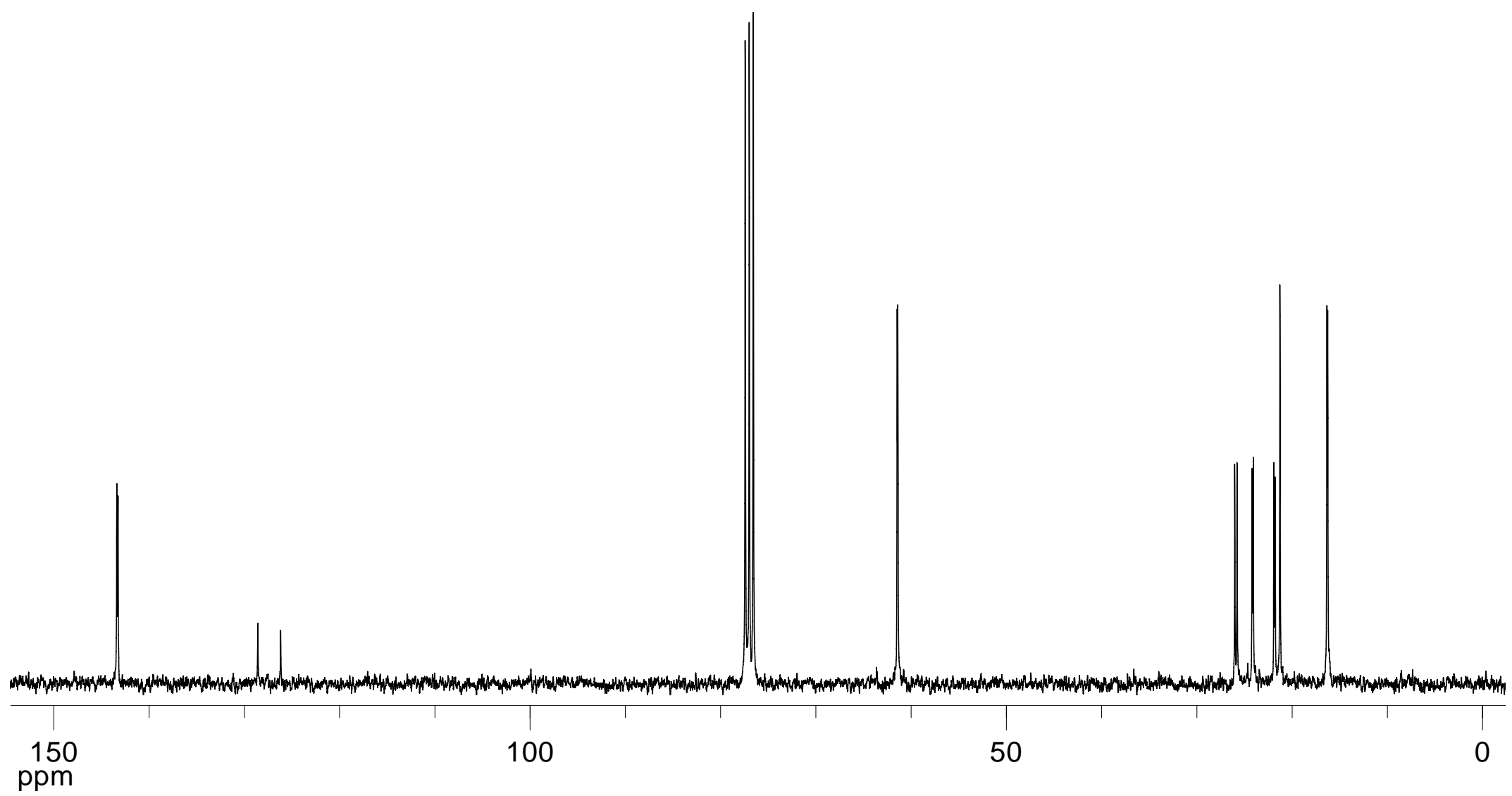




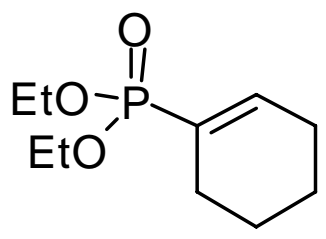

$25 a$

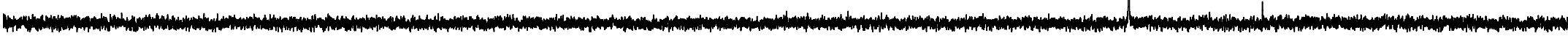

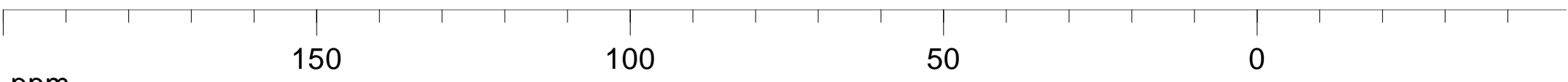

ppm 


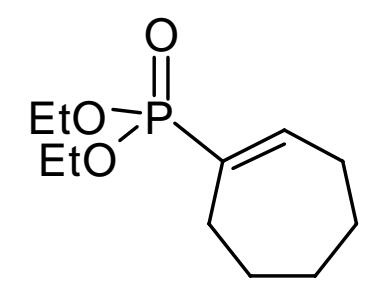

$18 a$

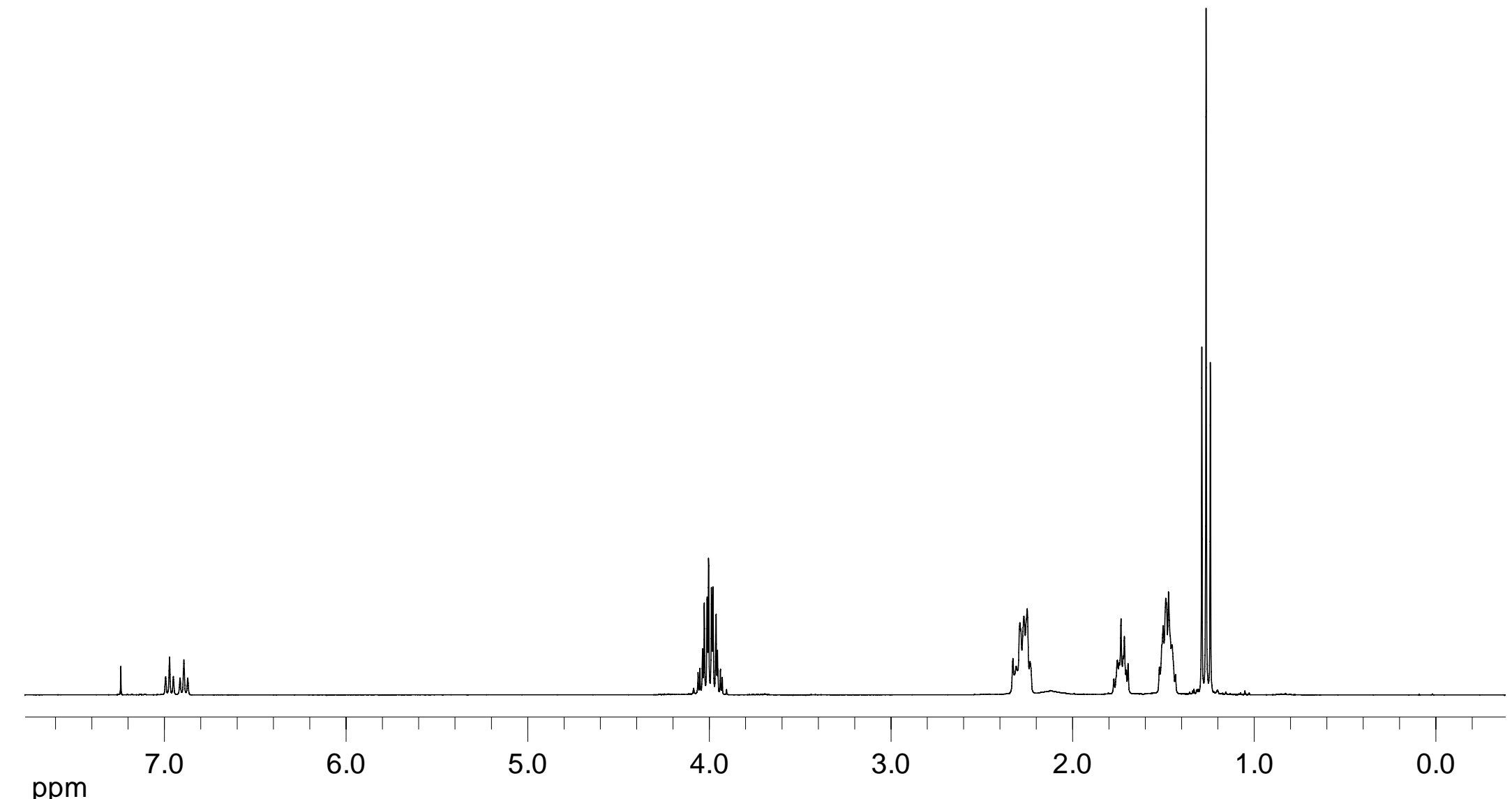




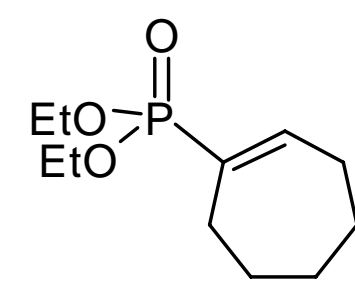

$18 a$

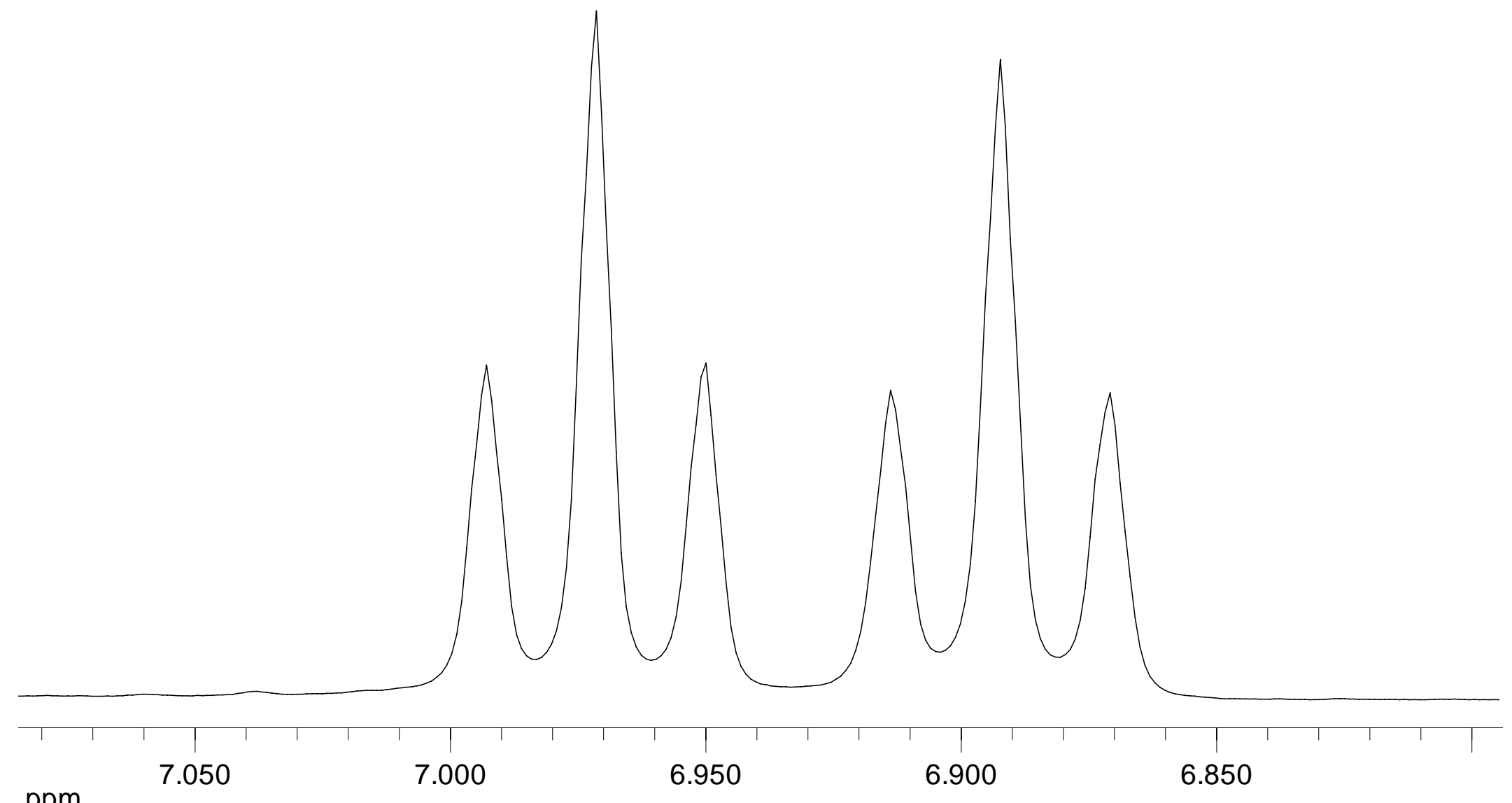




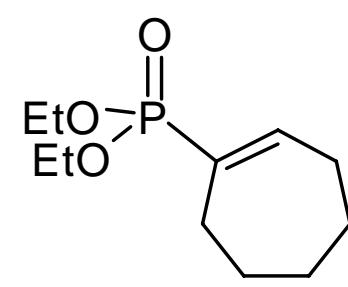

$18 a$

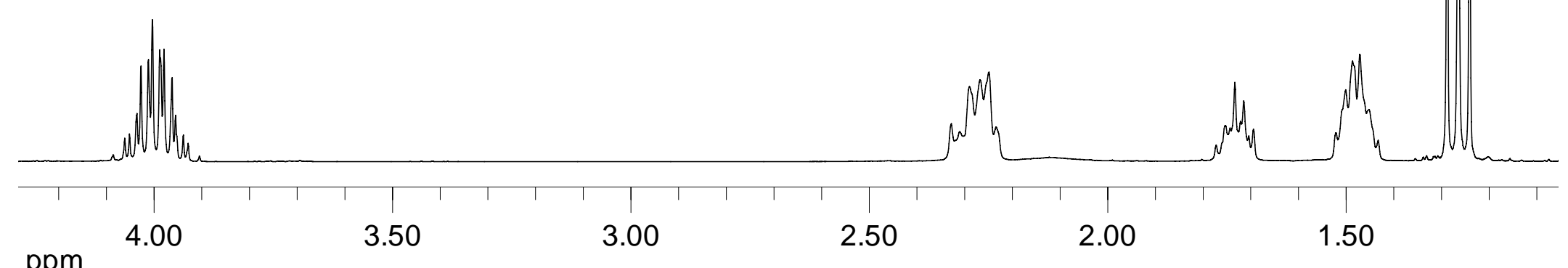




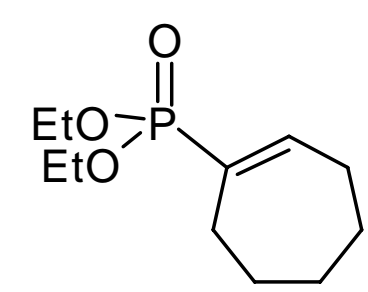

$18 a$

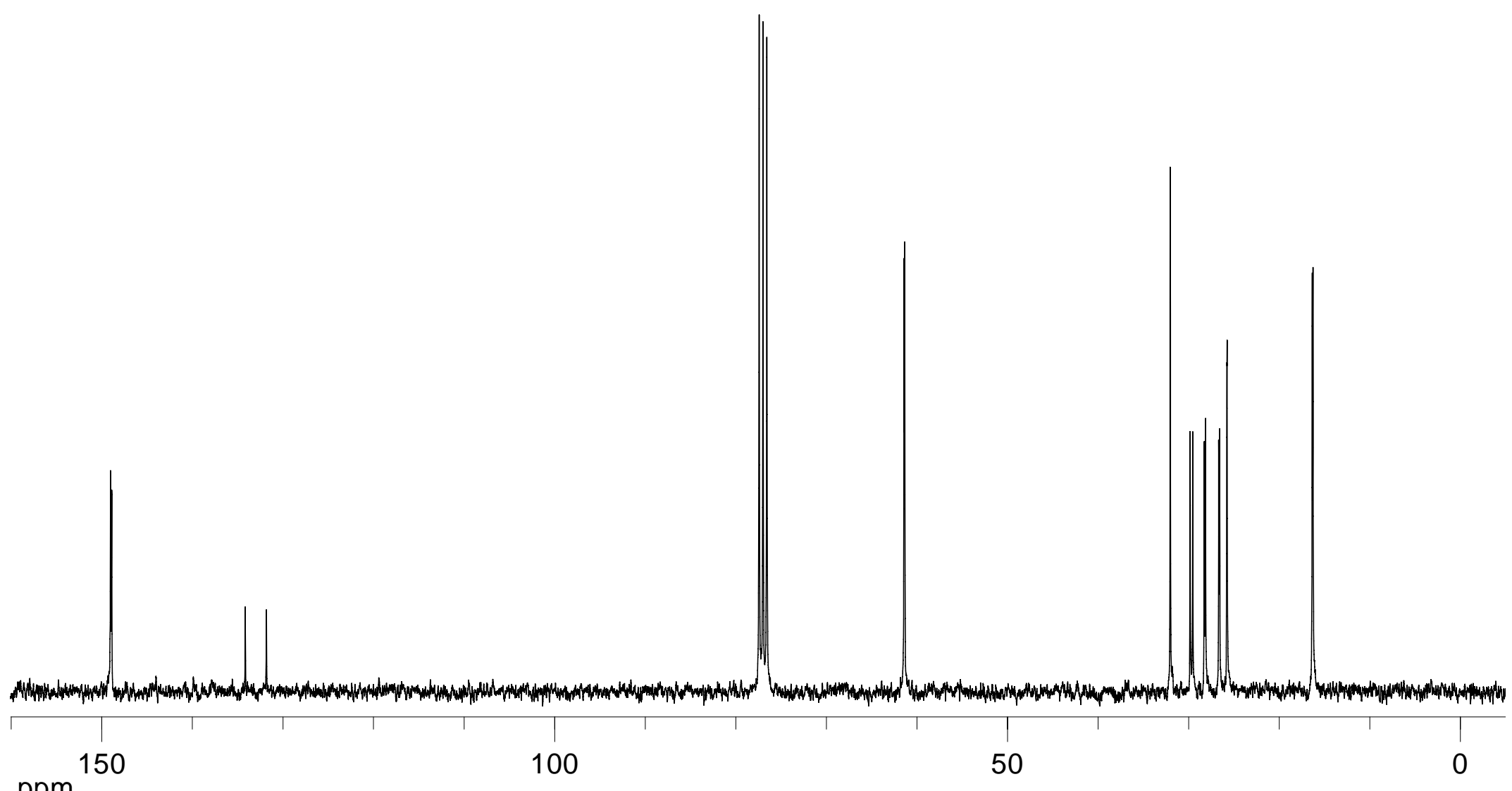

ppm 


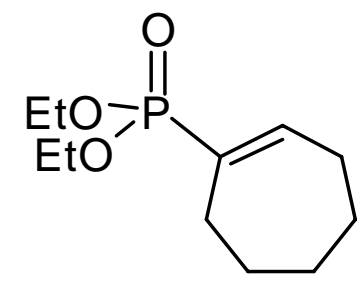

$18 a$

(1)

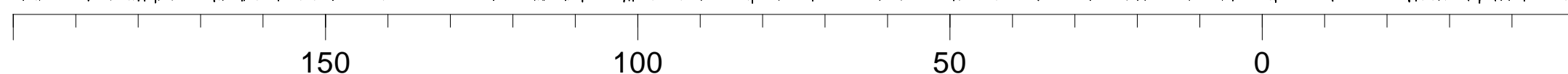

ppm

150

100

50 


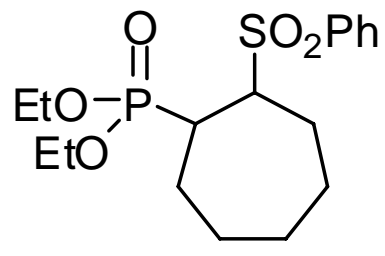

Phosphonate adduct of $\mathbf{1 8}$

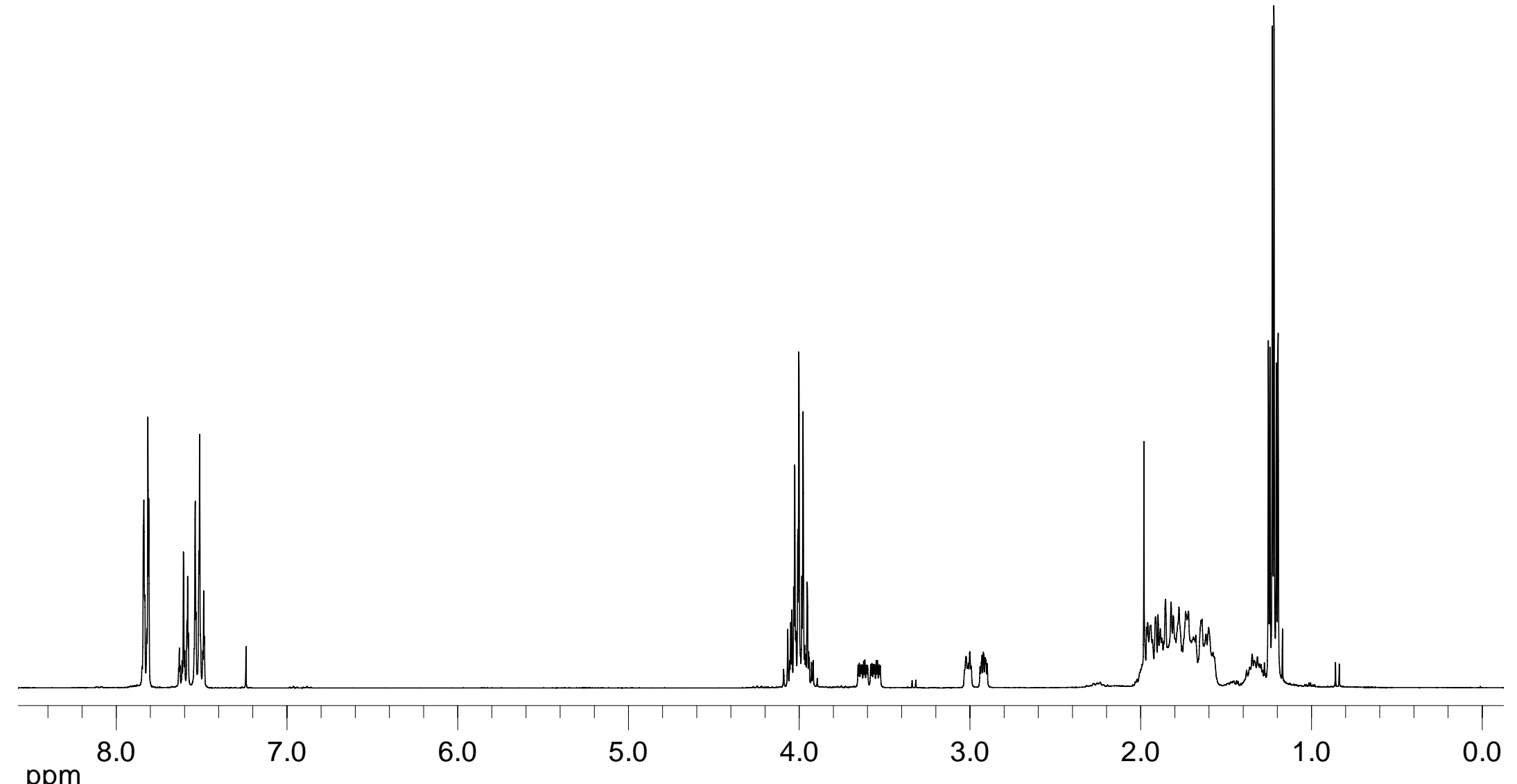




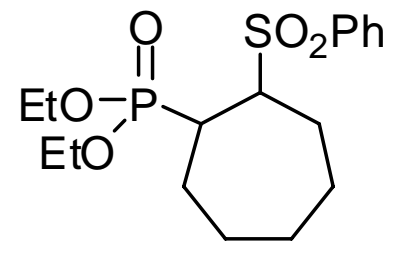

Phosphonate adduct of $\mathbf{1 8}$

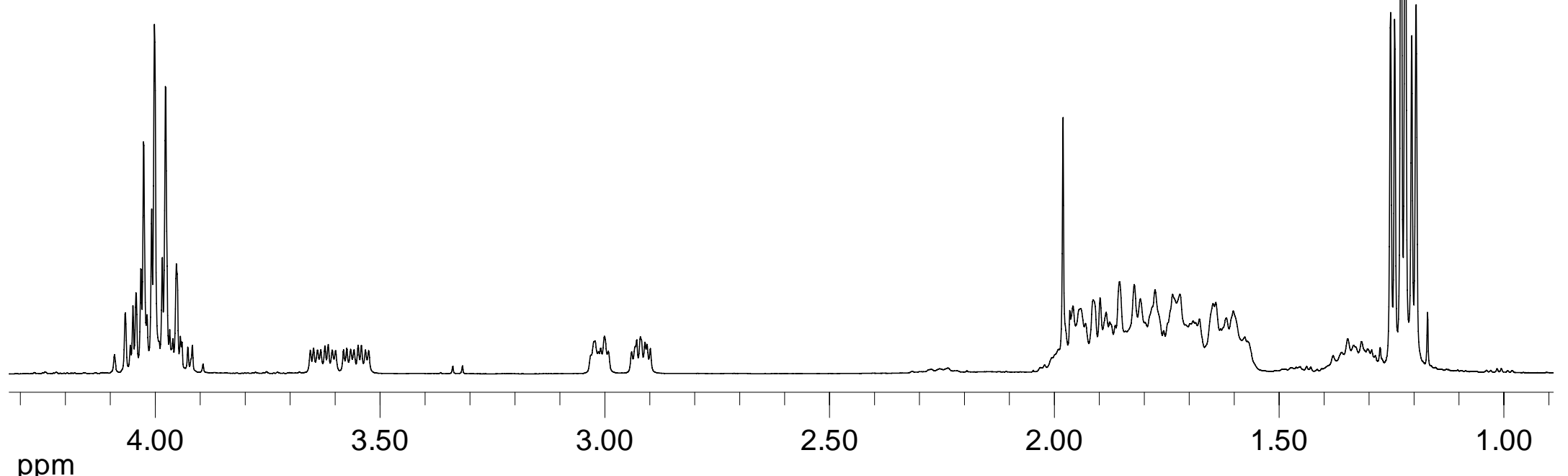




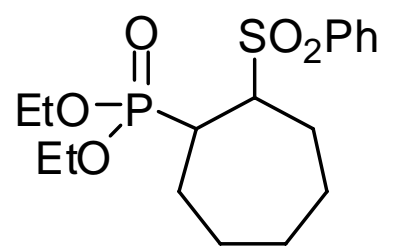

Phosphonate adduct of $\mathbf{1 8}$

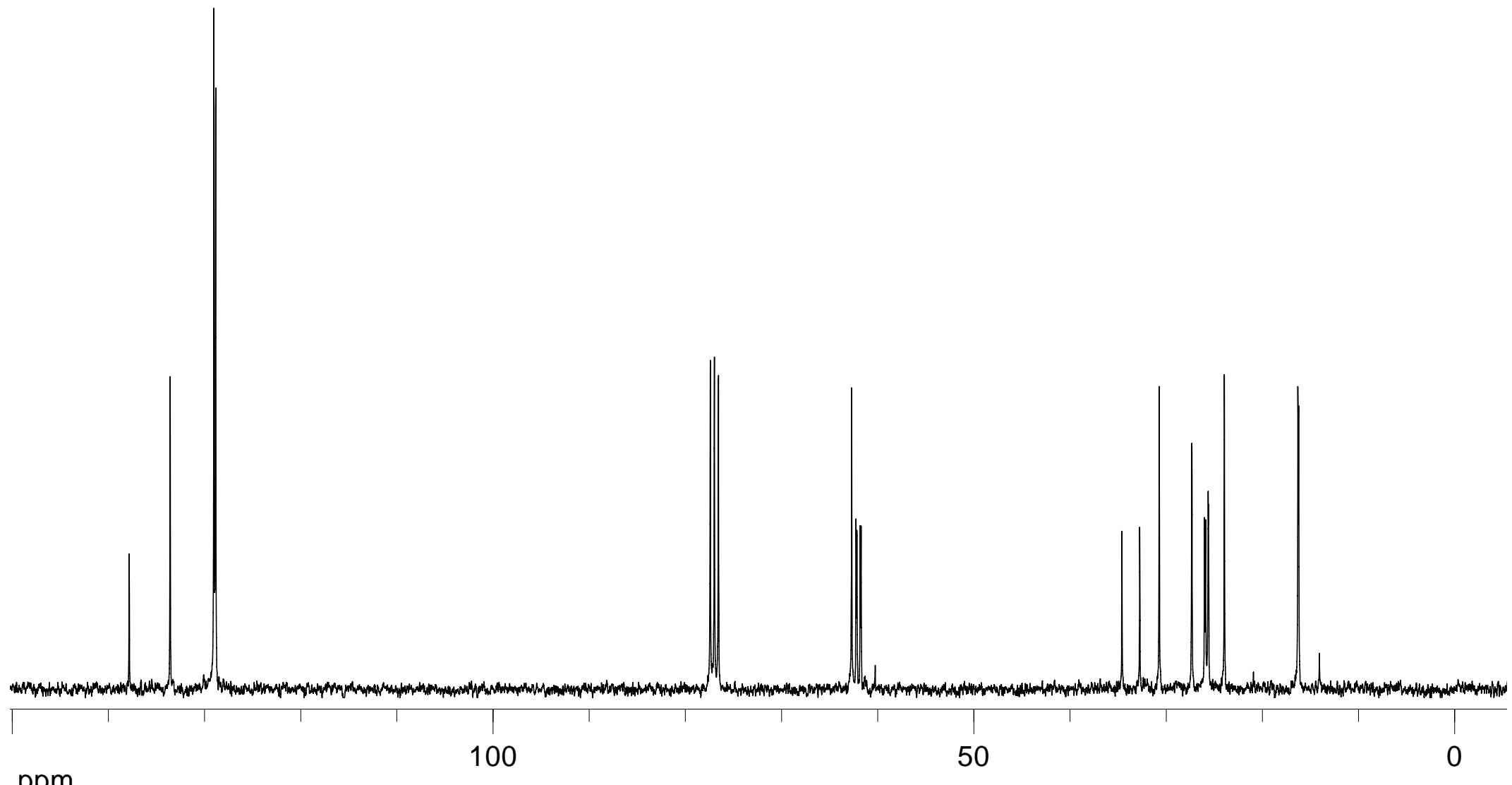

ppm 


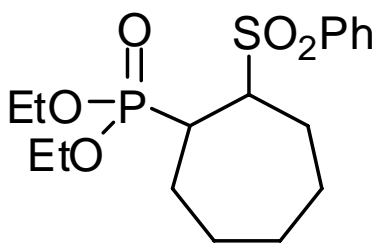

Phosphonate adduct of $\mathbf{1 8}$ 

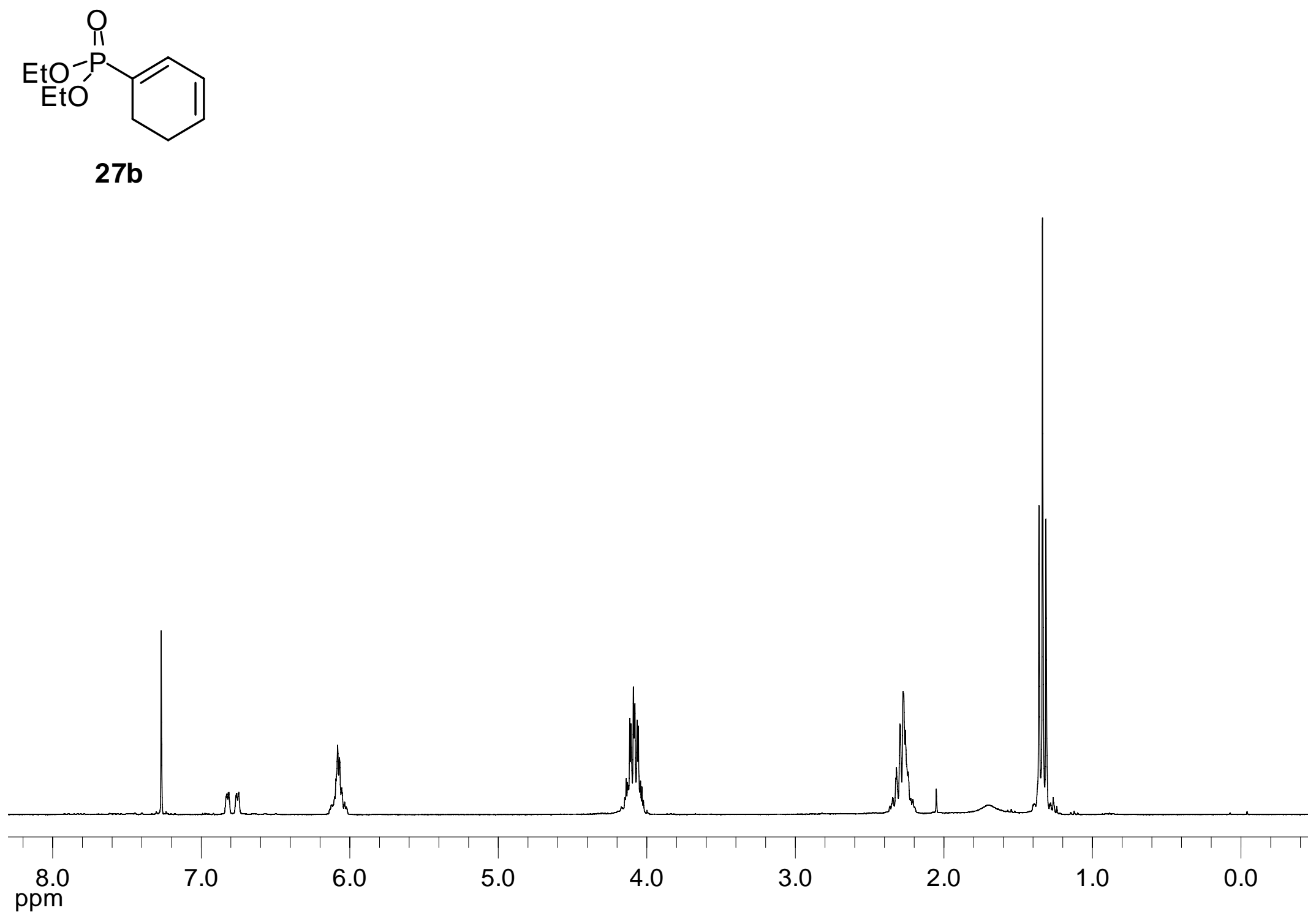


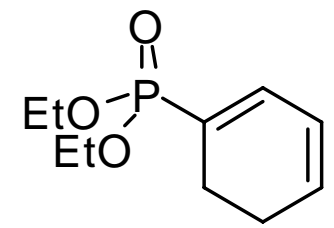

27b

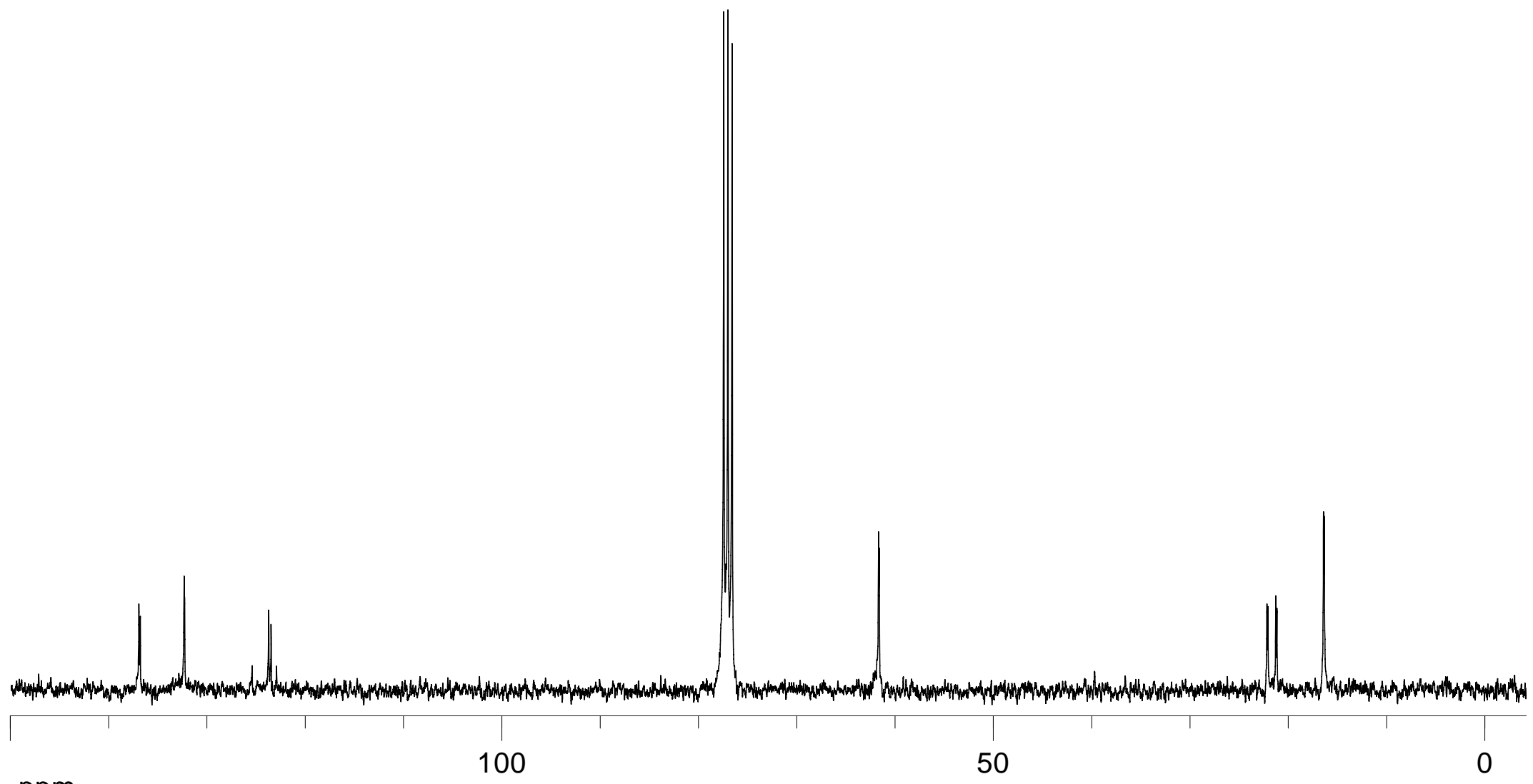

ppm 

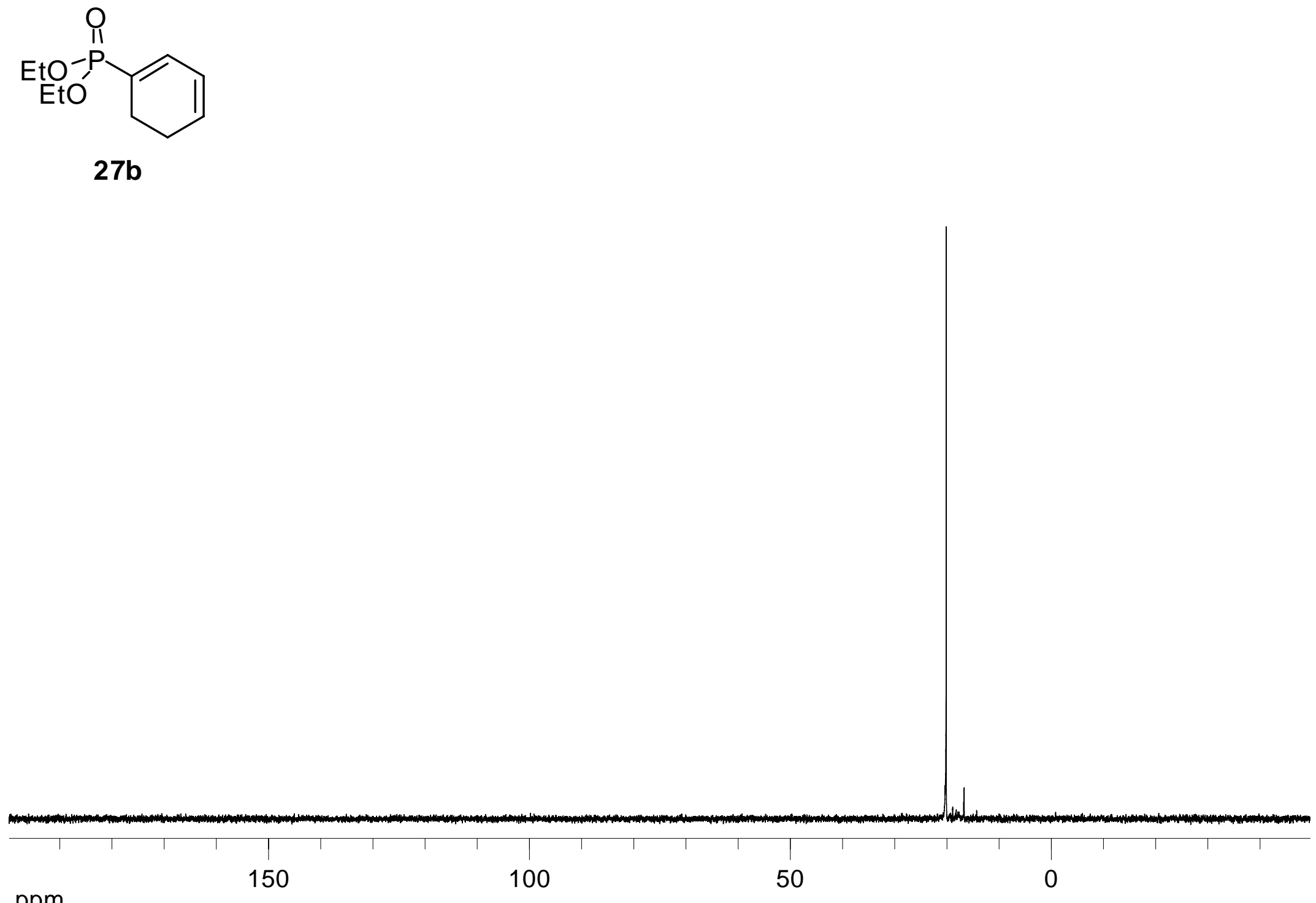

ppm 

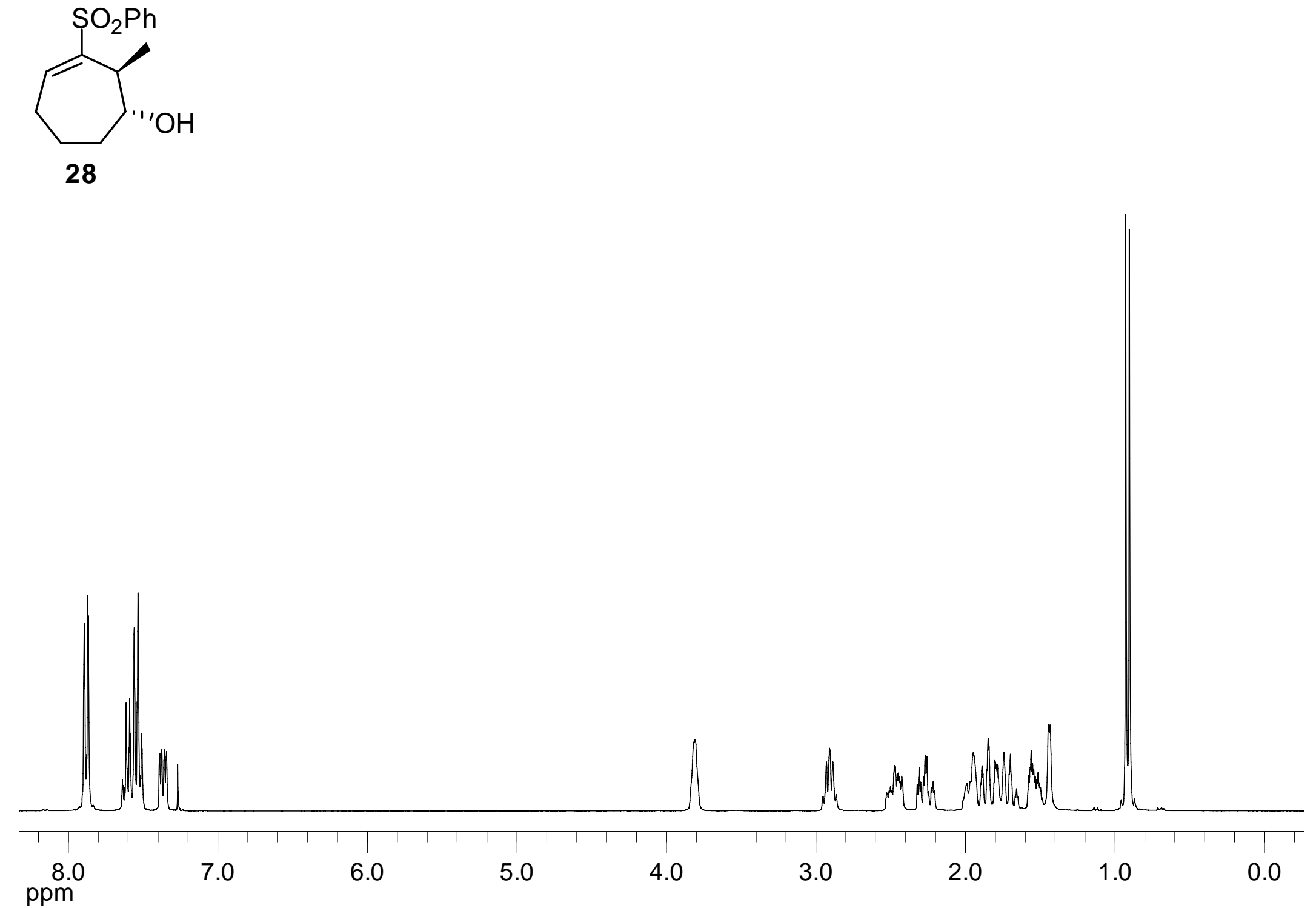


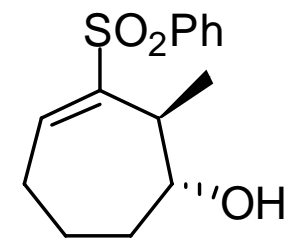

28

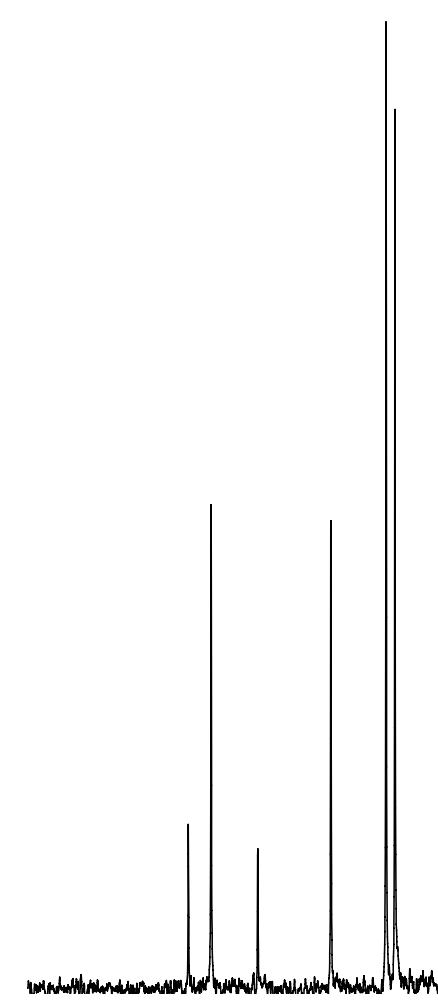

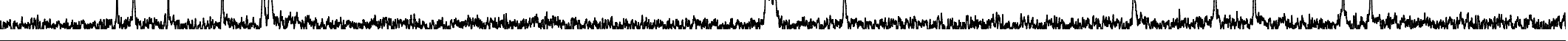




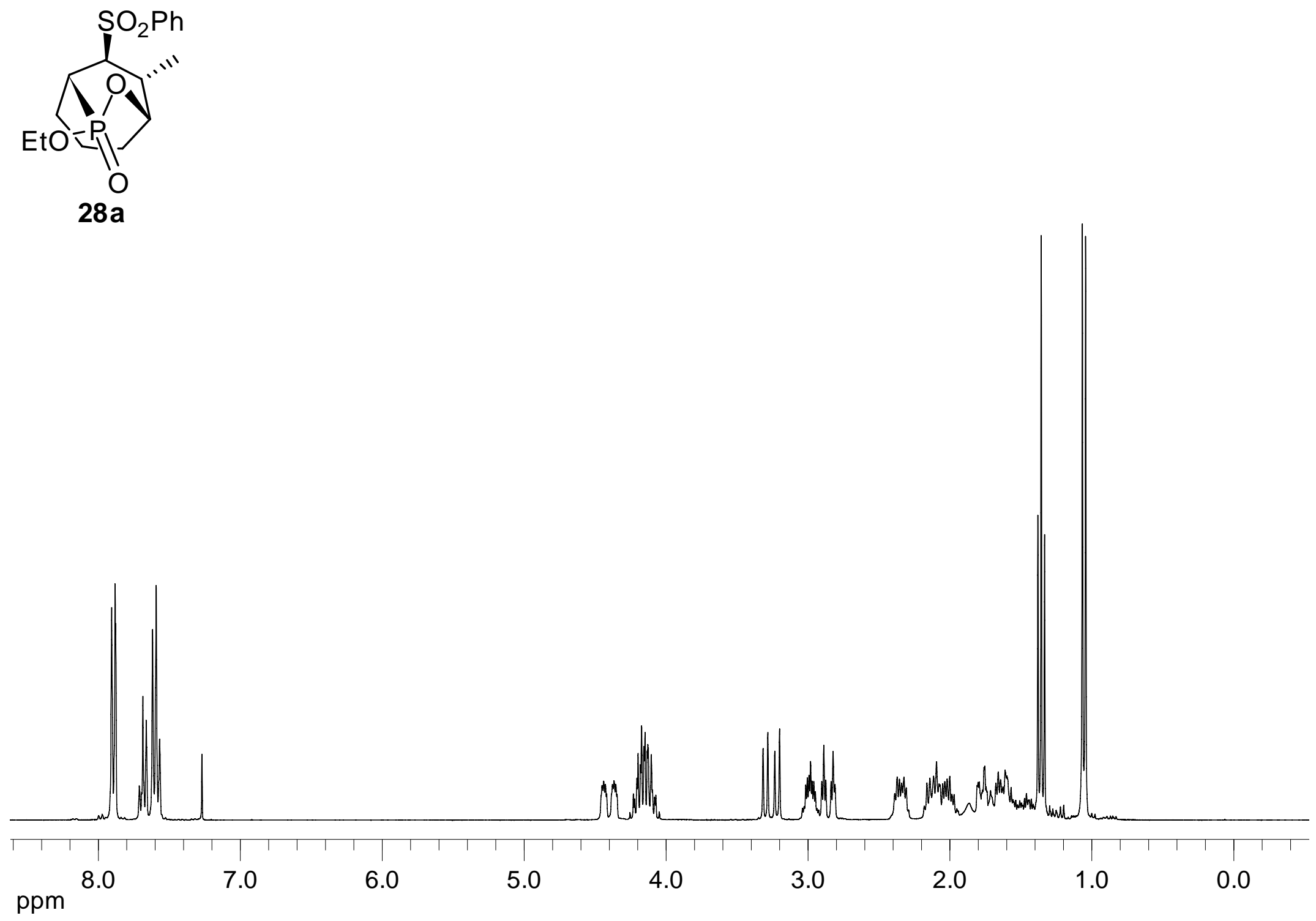



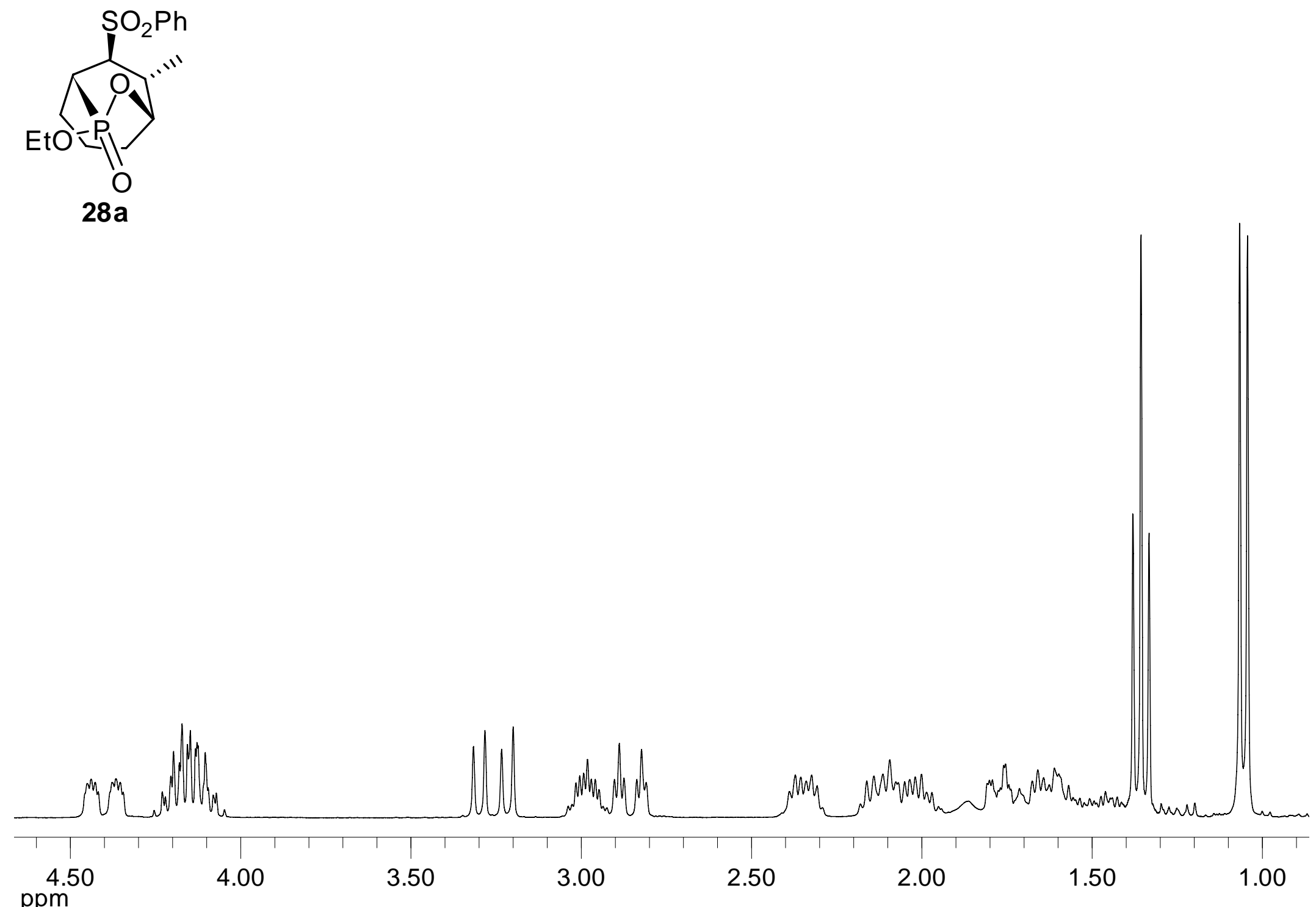


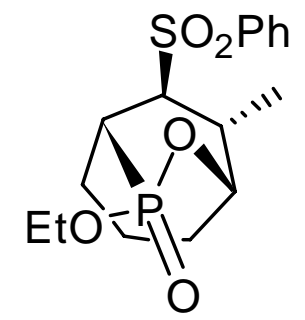

28a

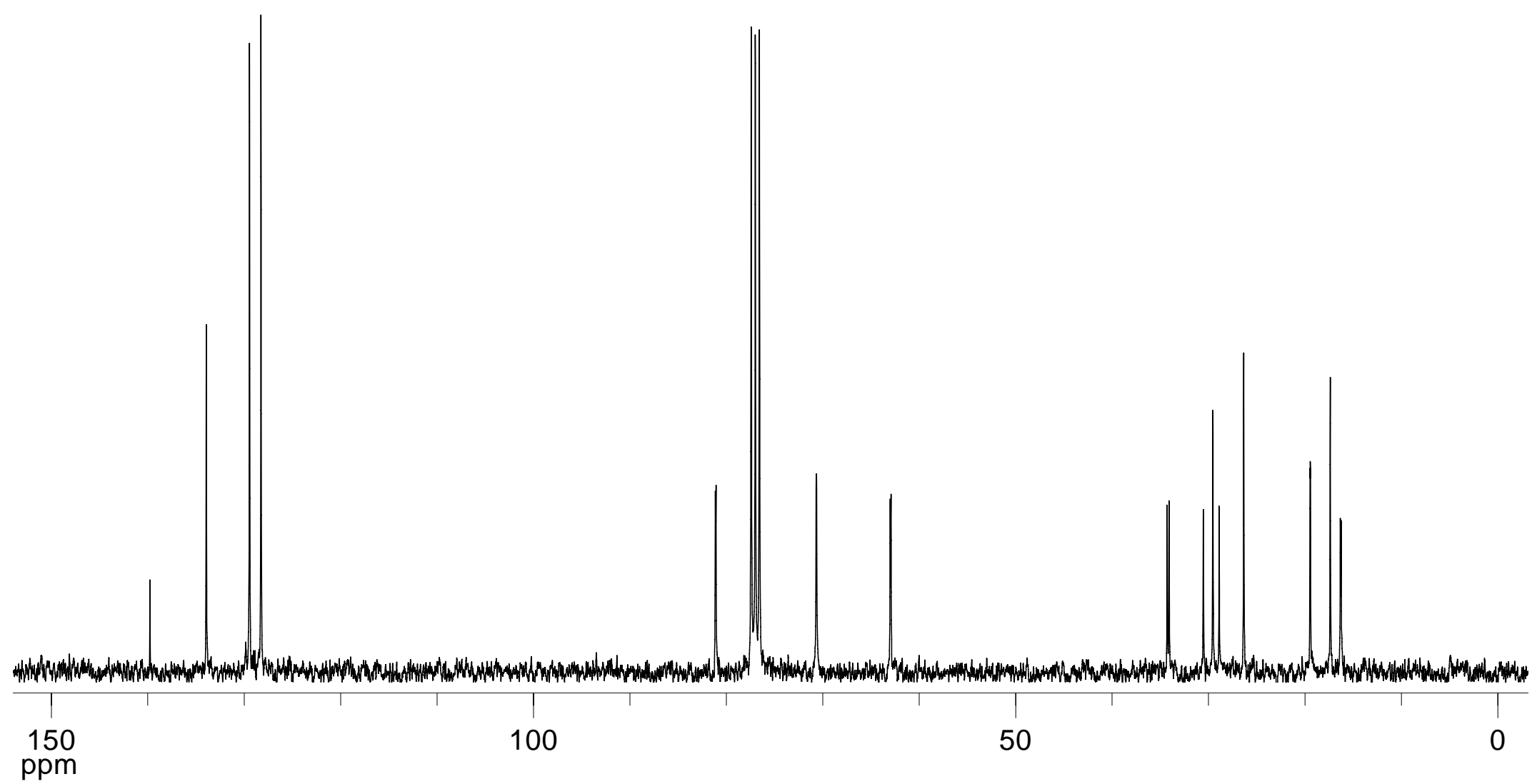



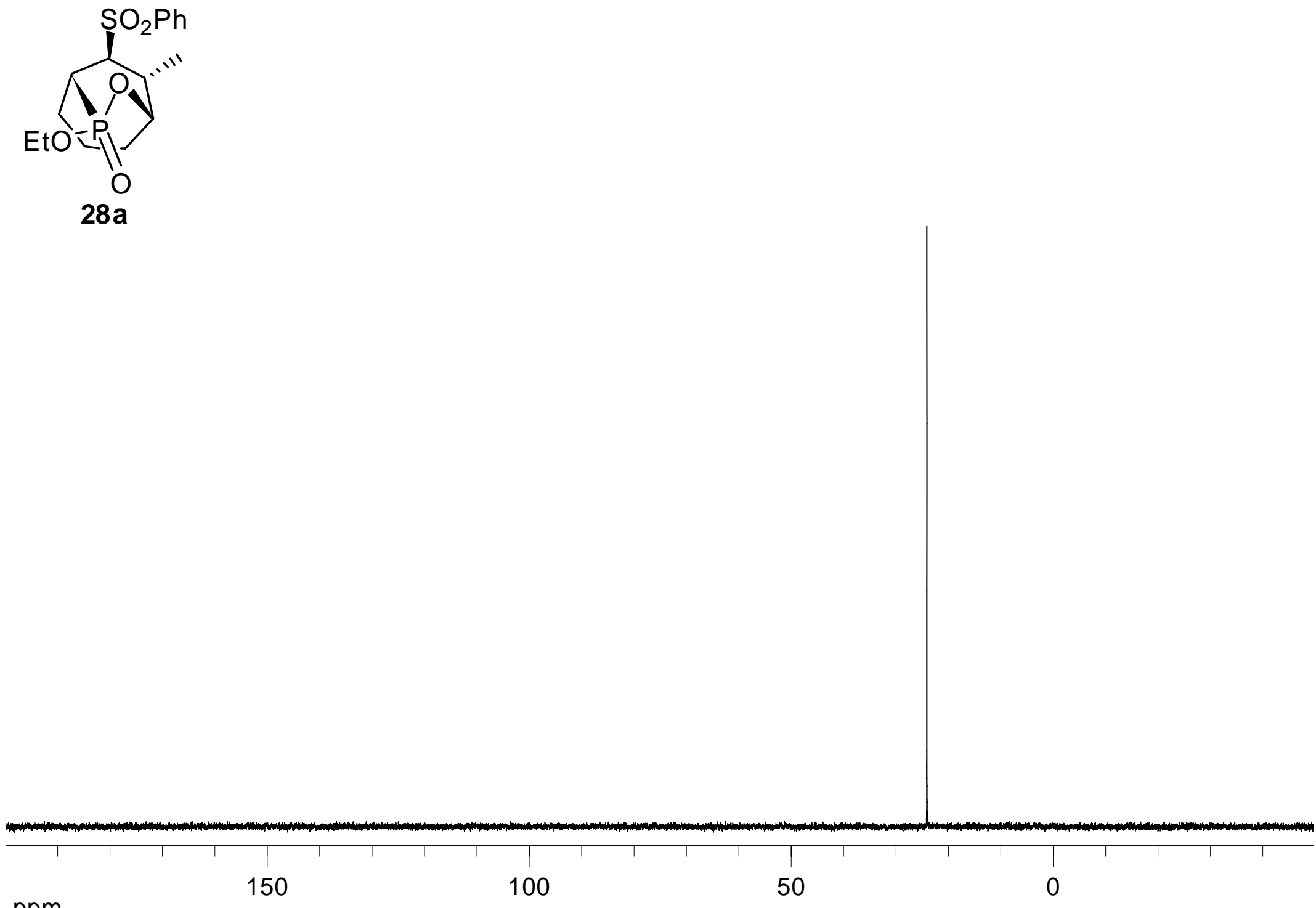

ppm 


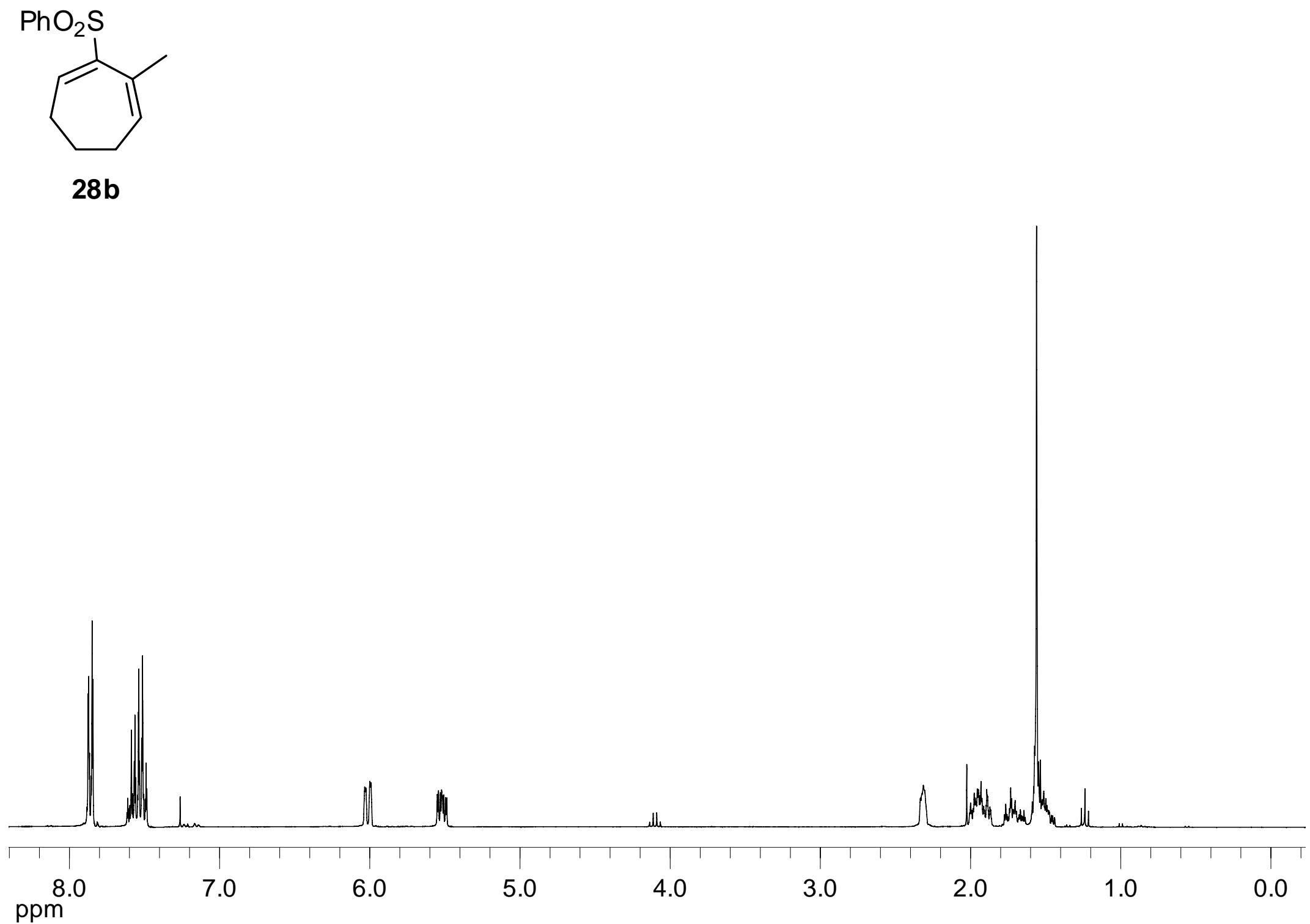




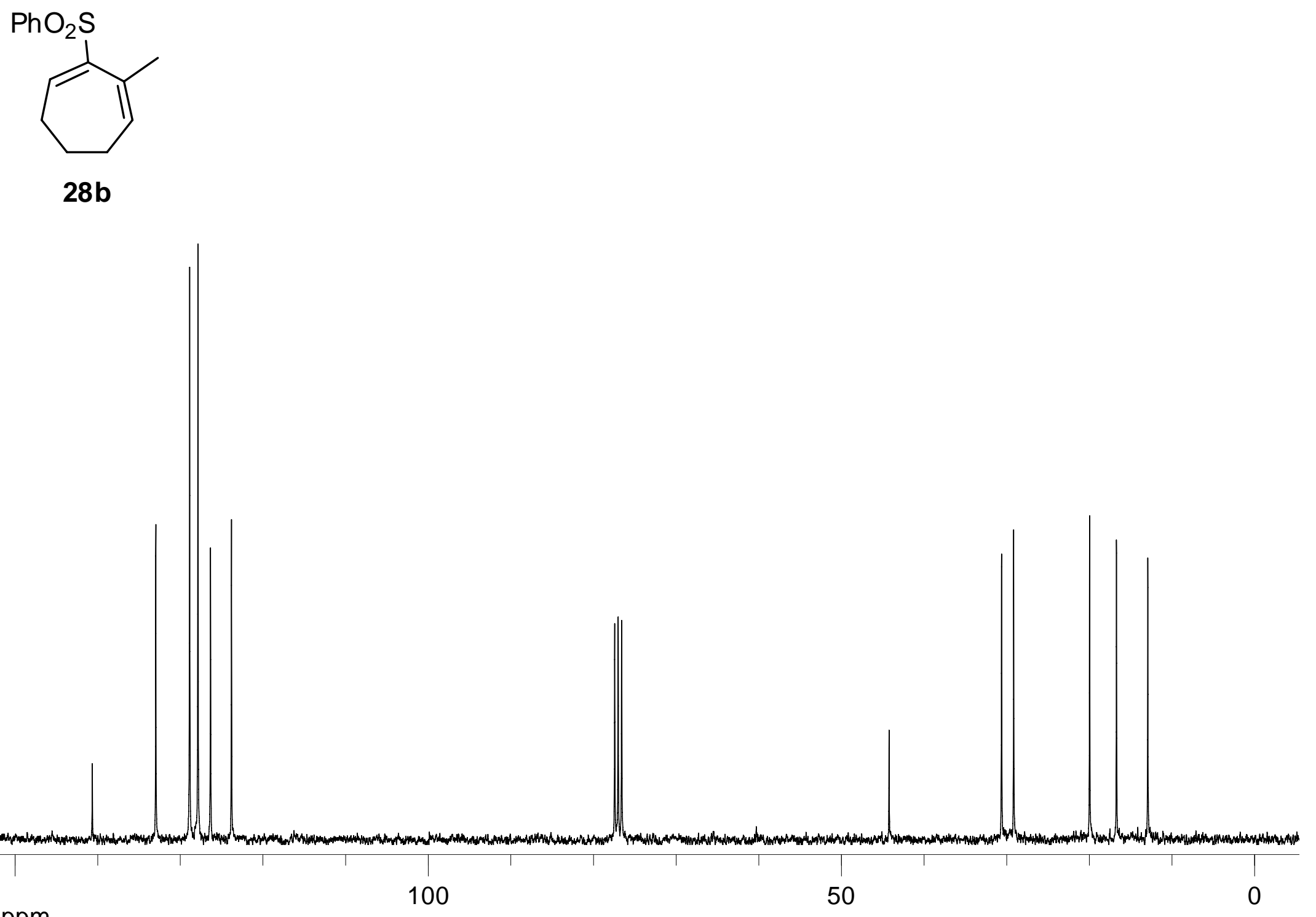

ppm 

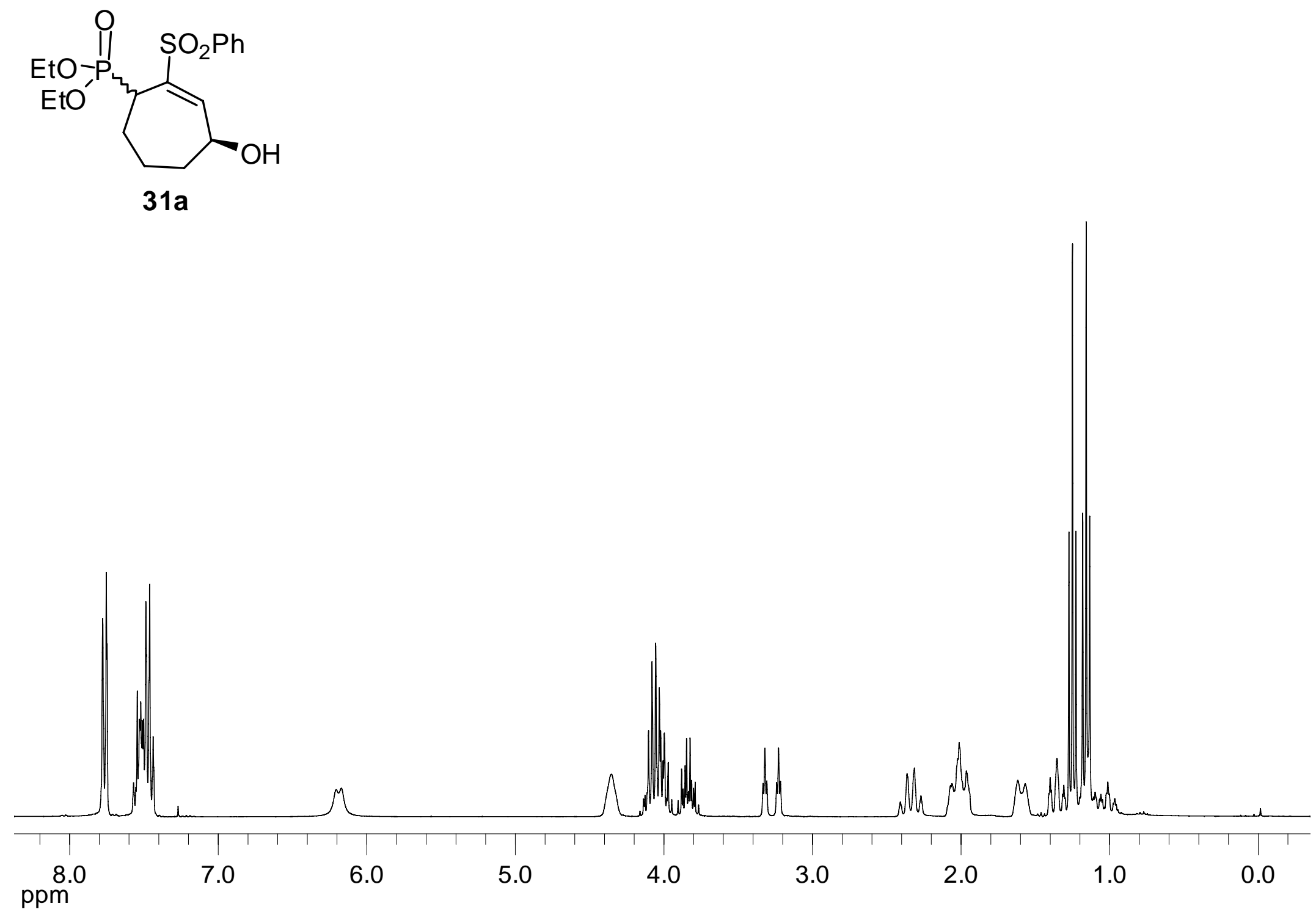

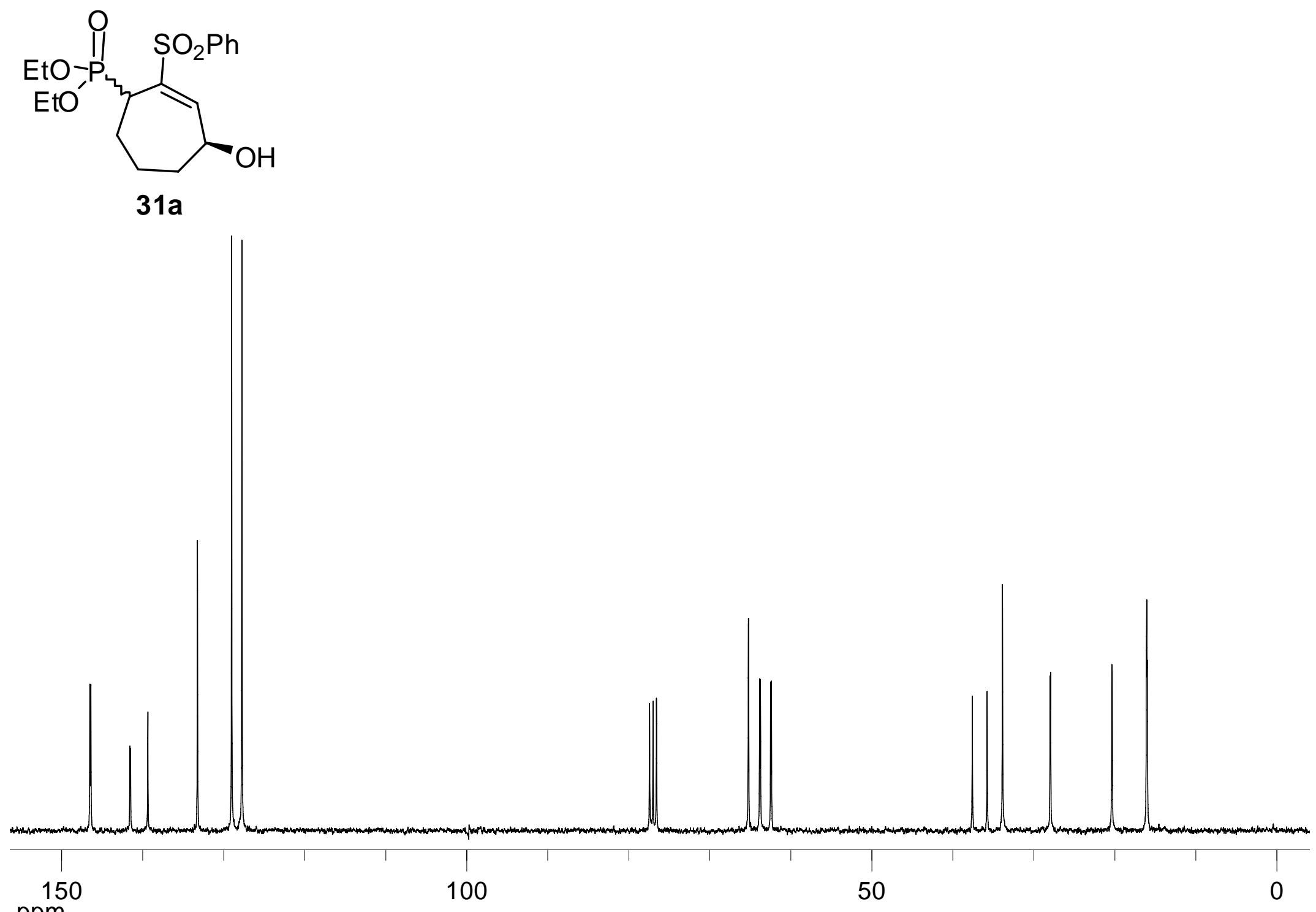

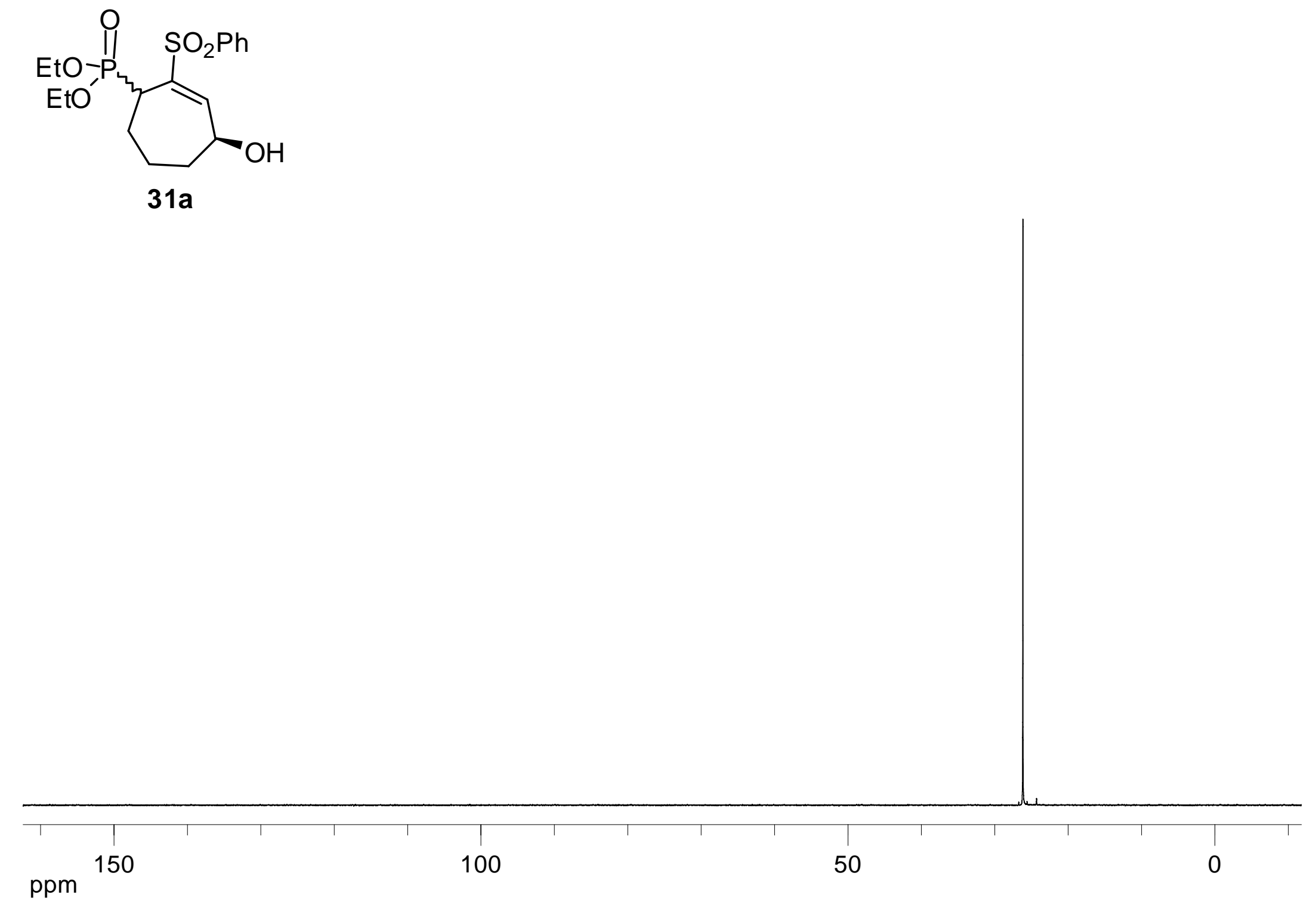


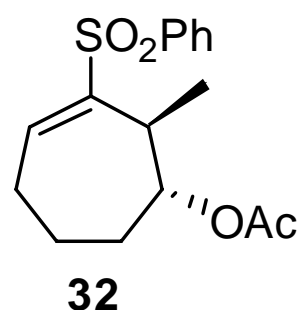

32

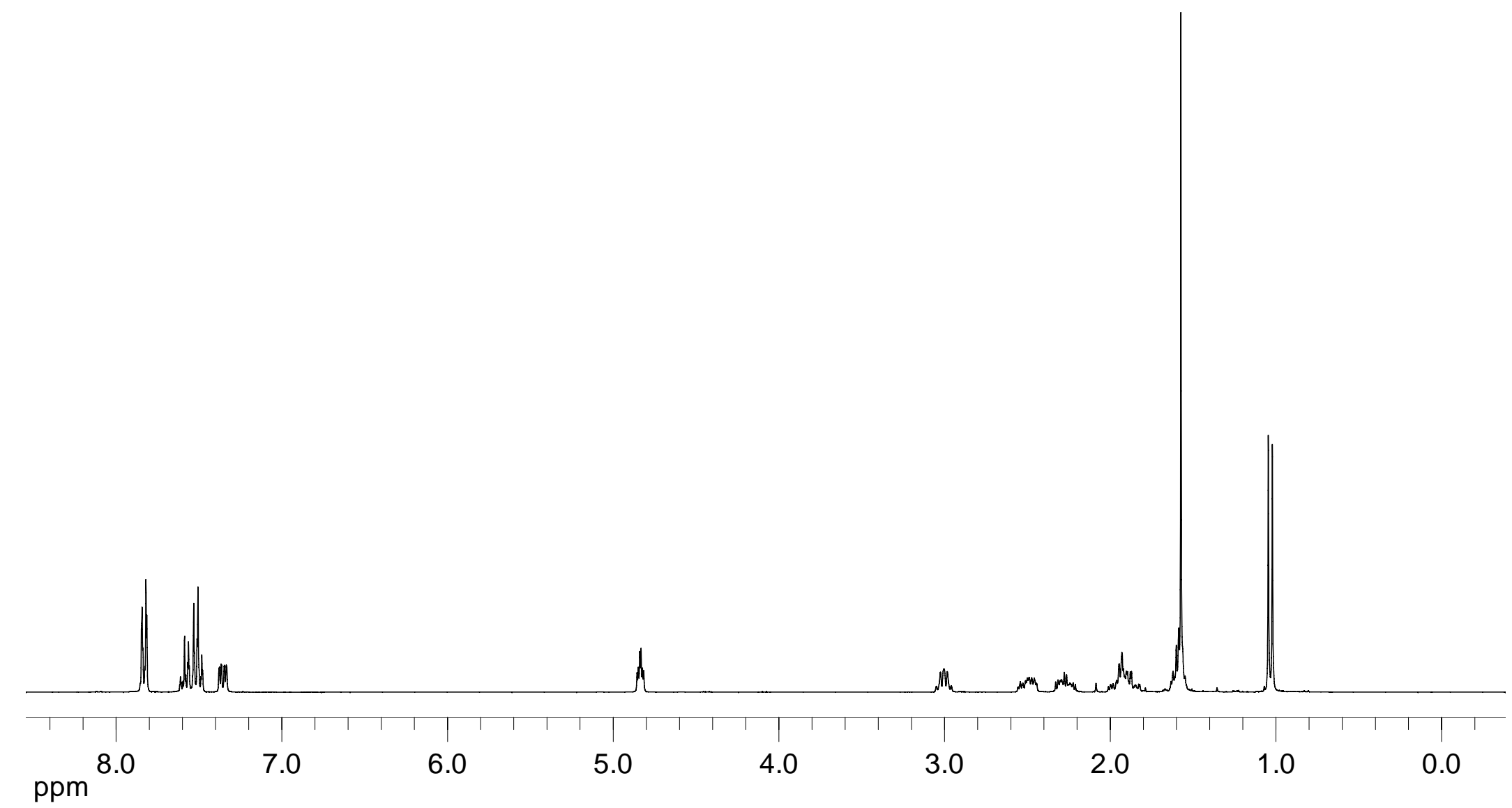



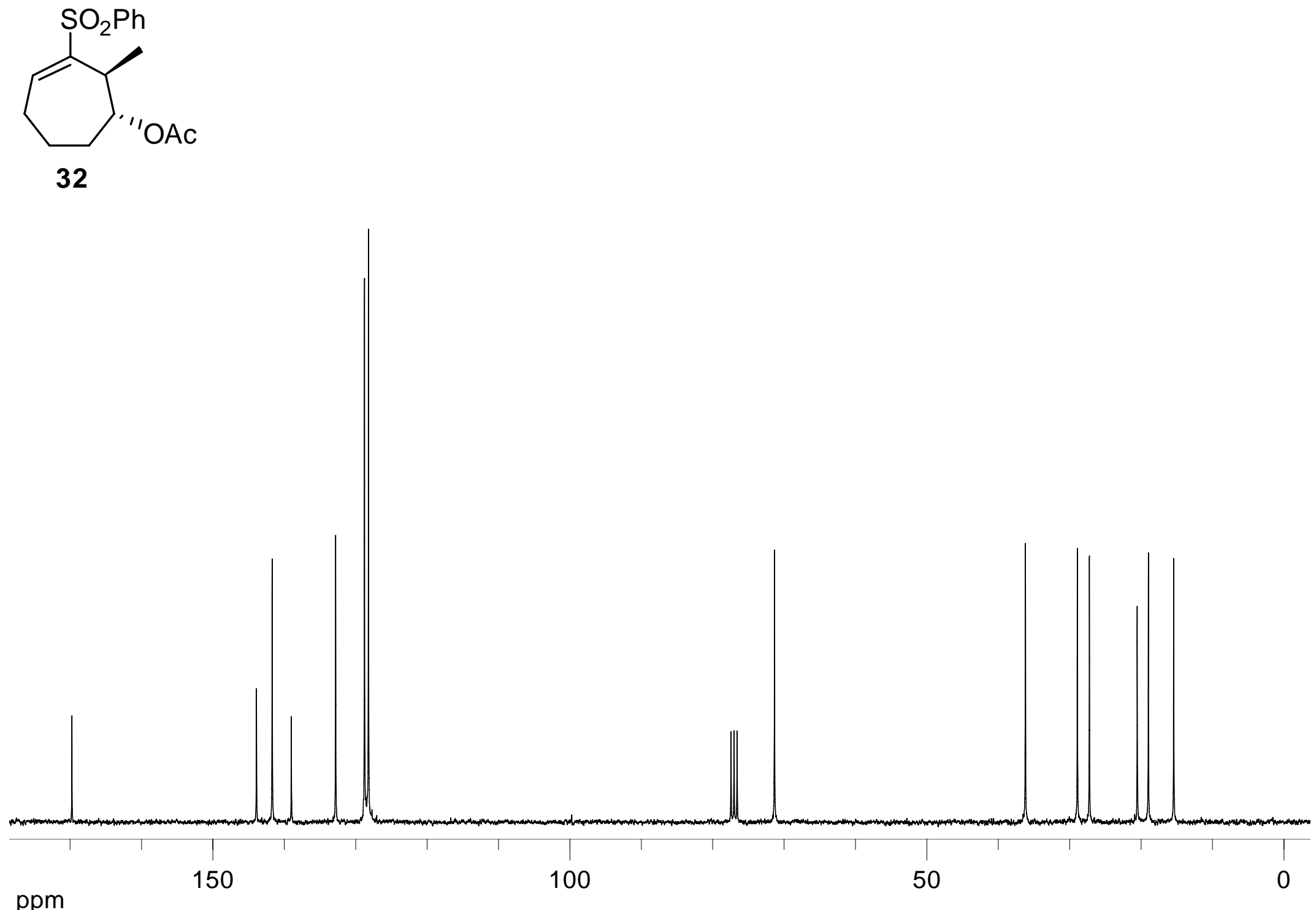

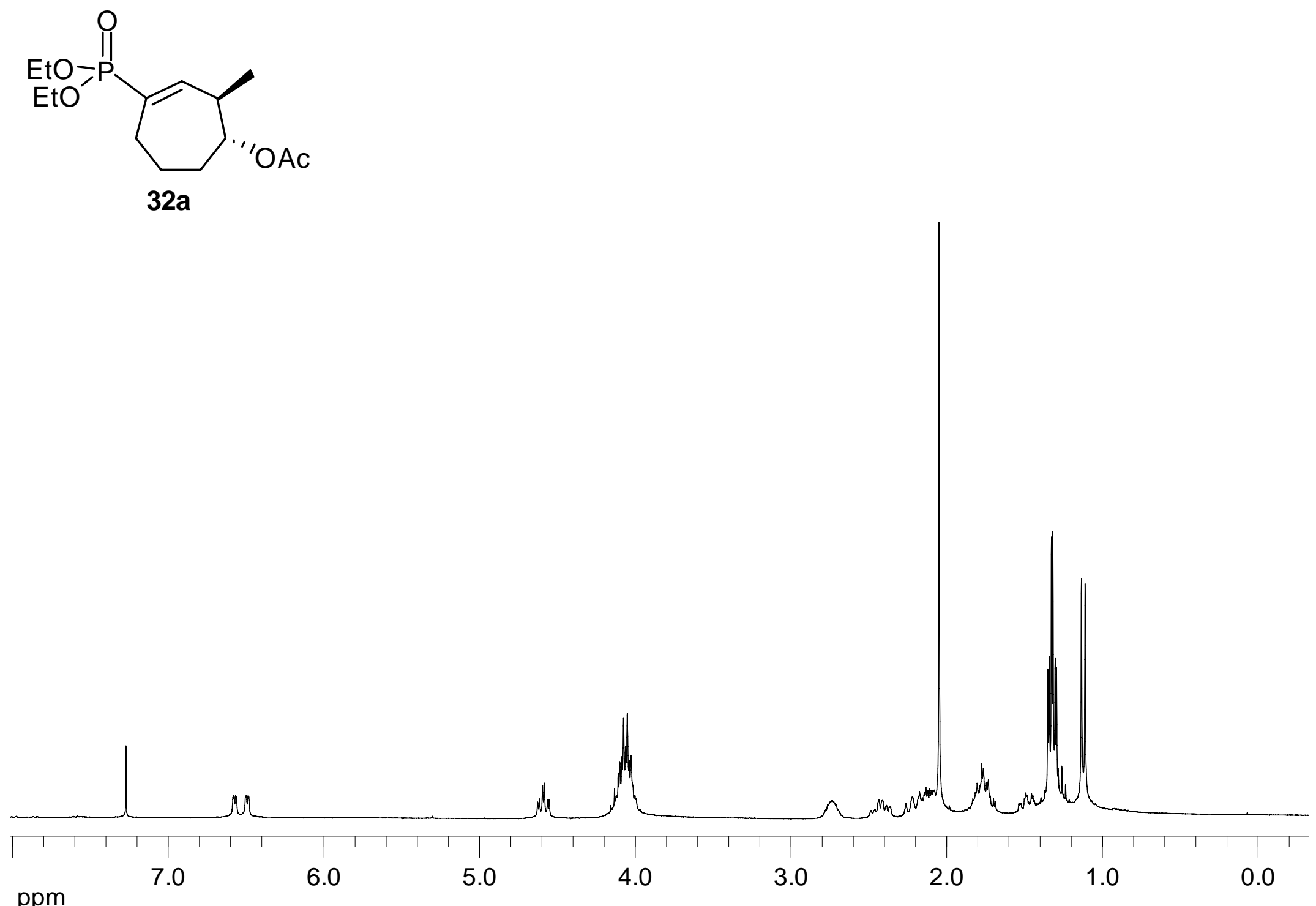


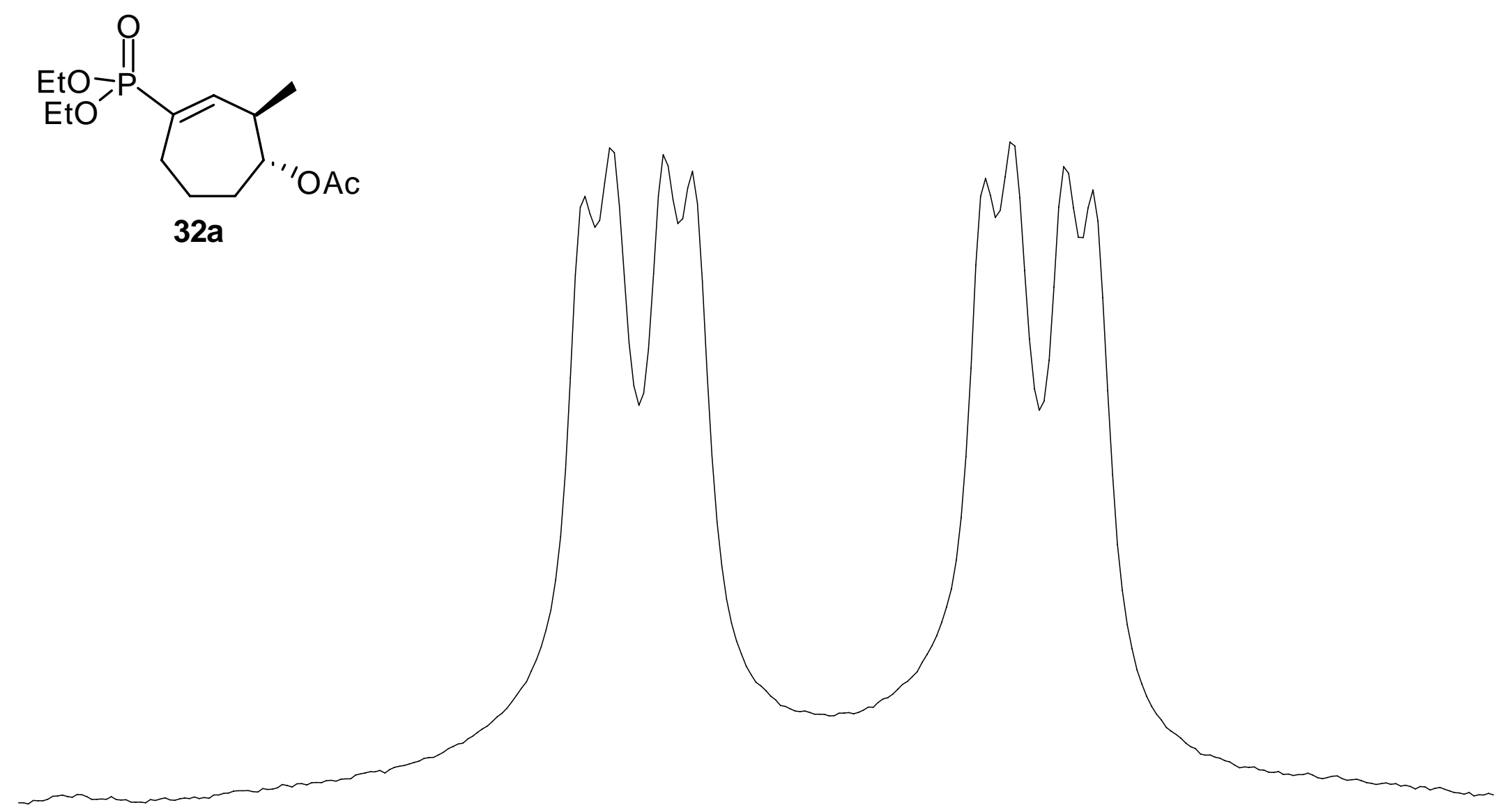

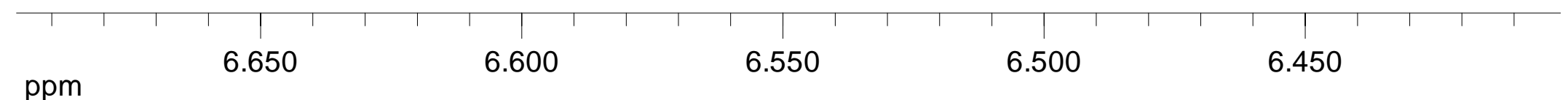



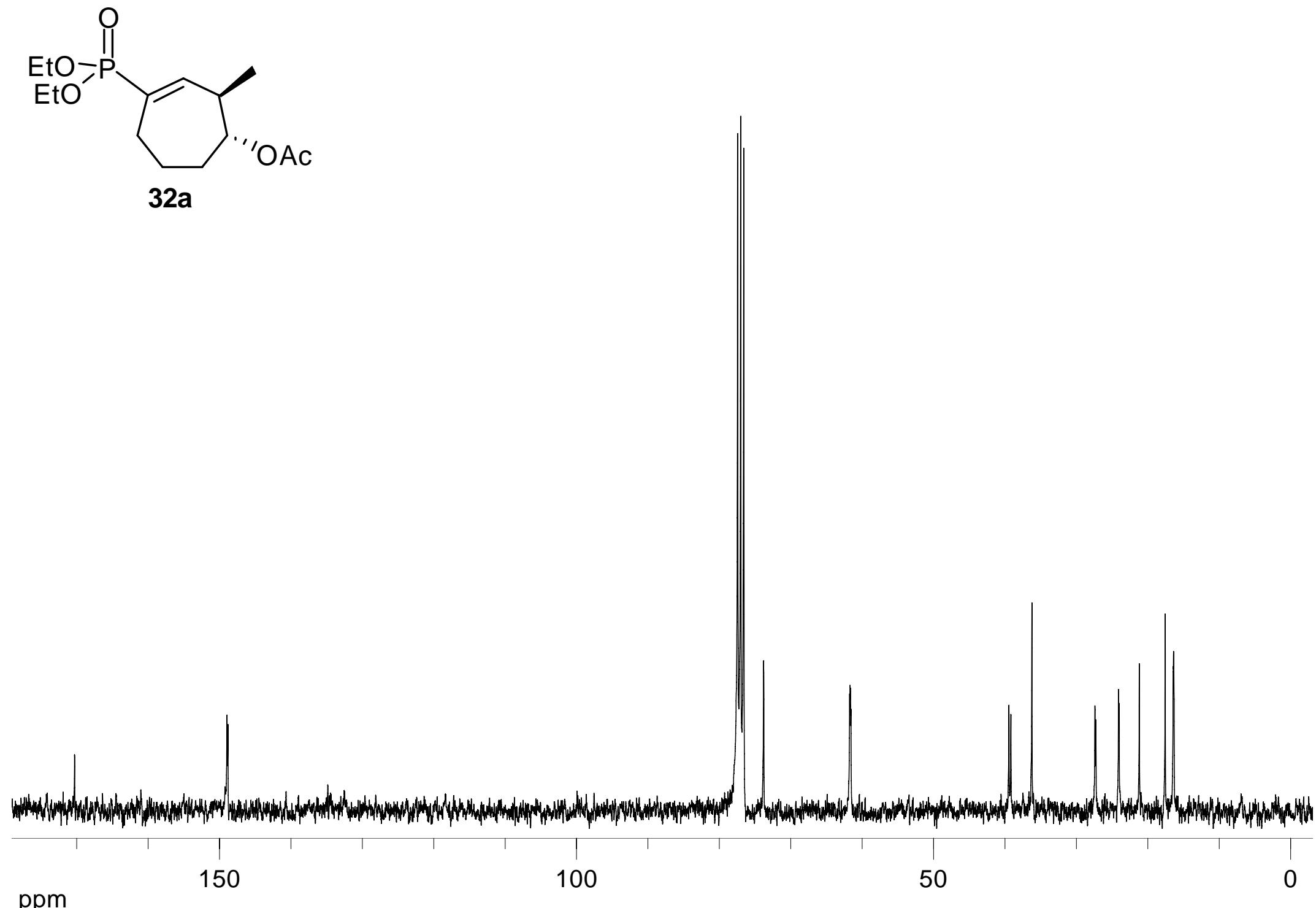

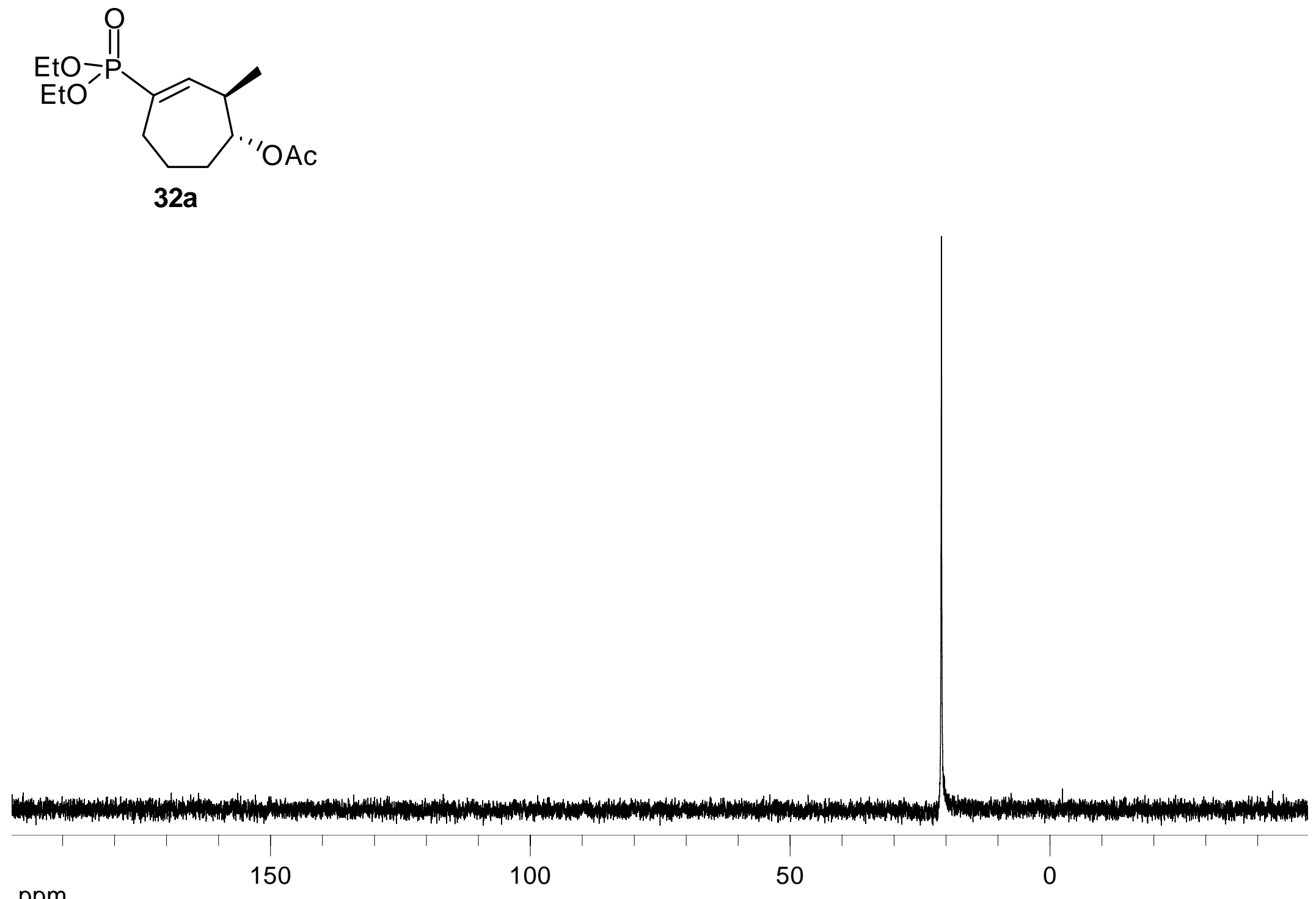


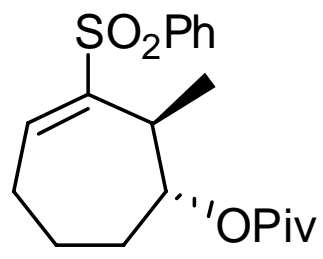

33

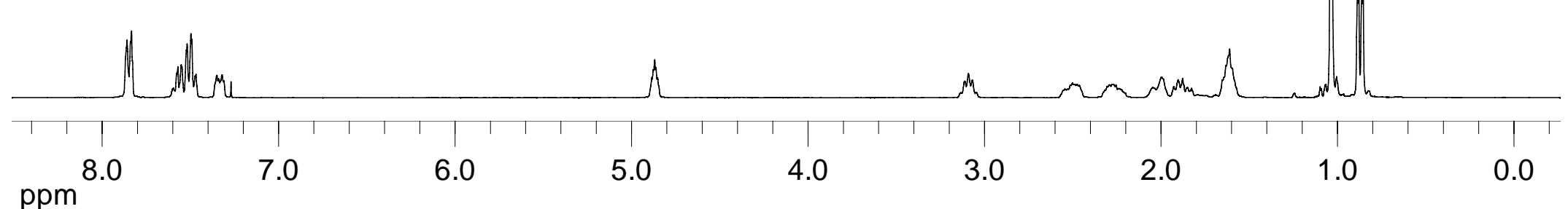




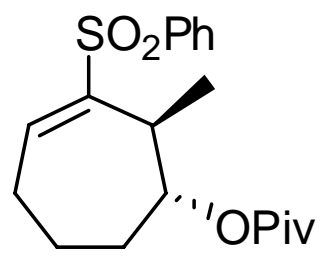

33

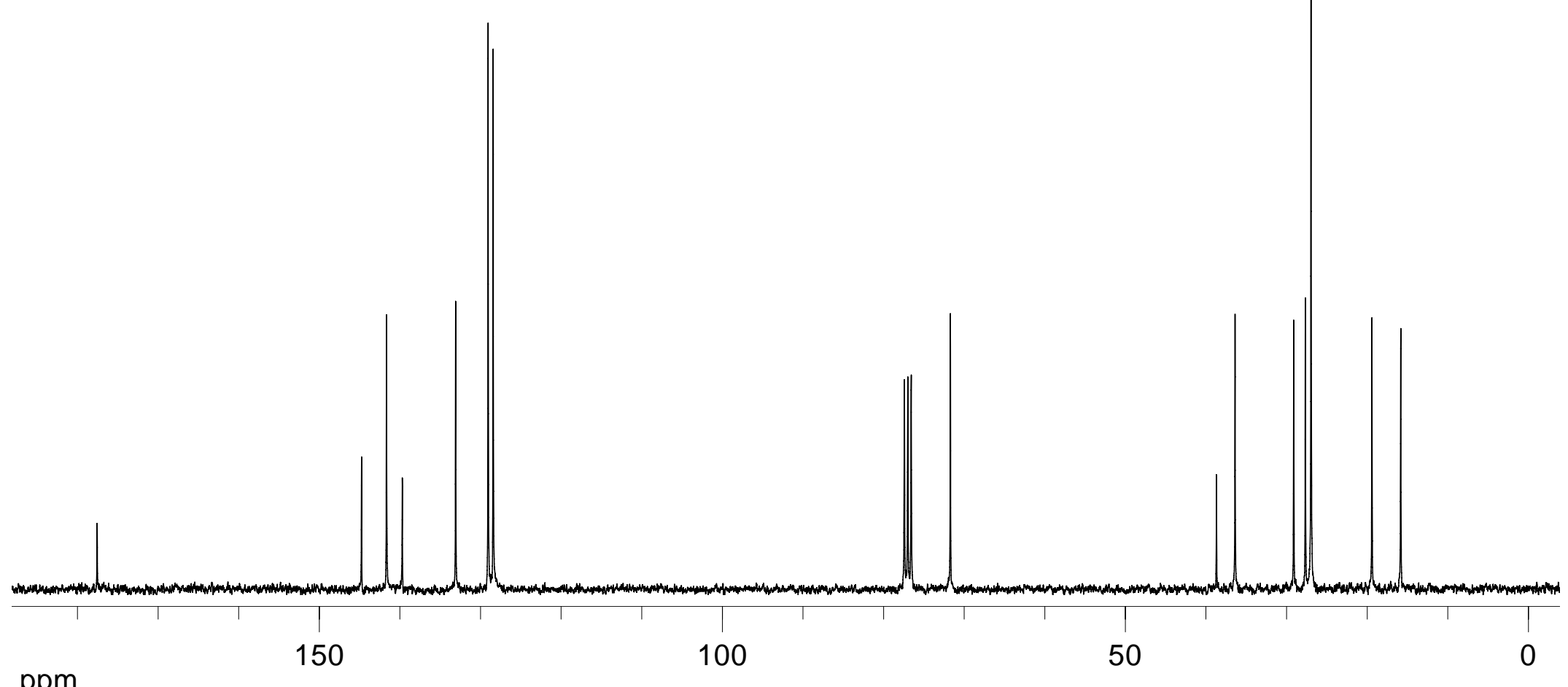

ppm 

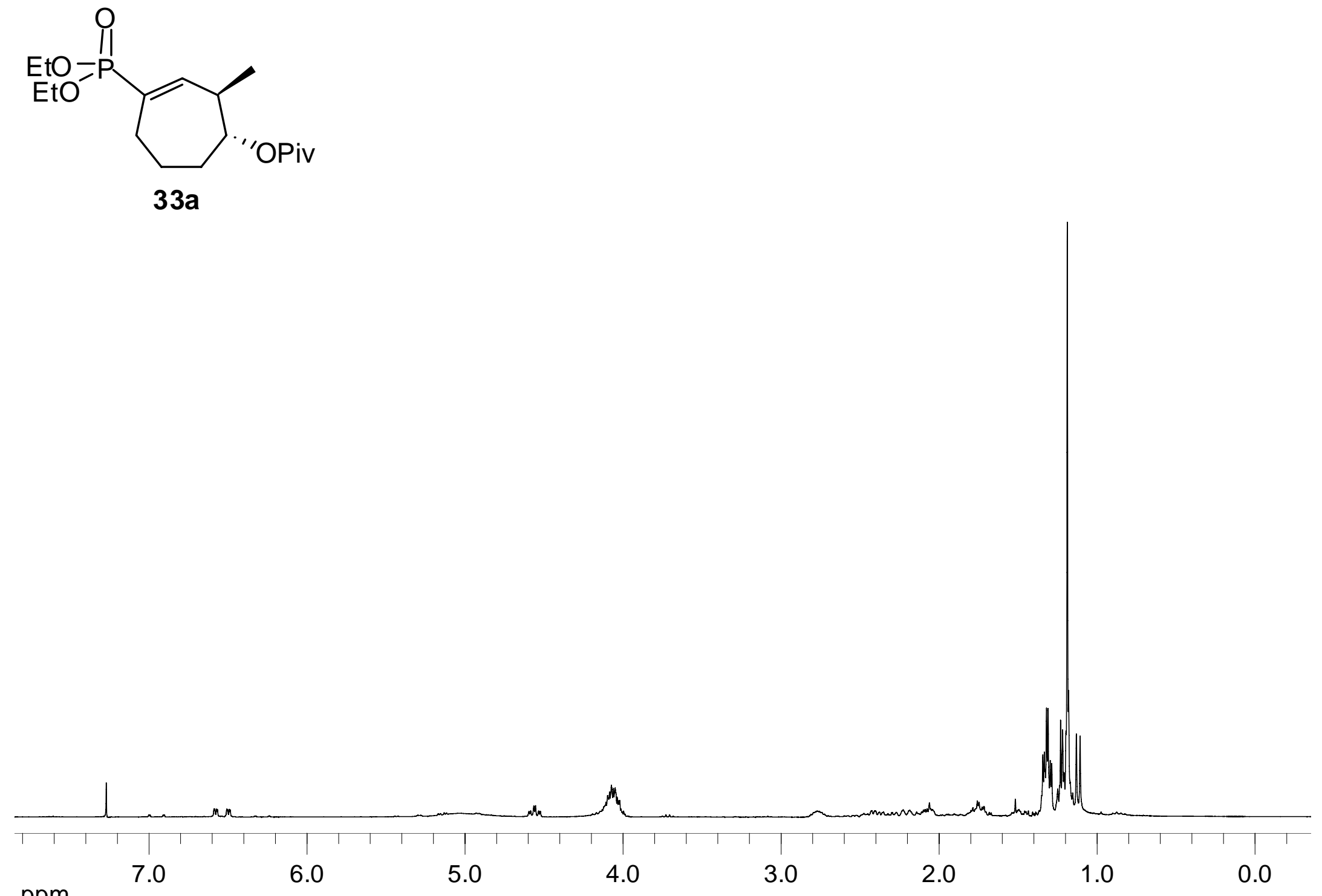


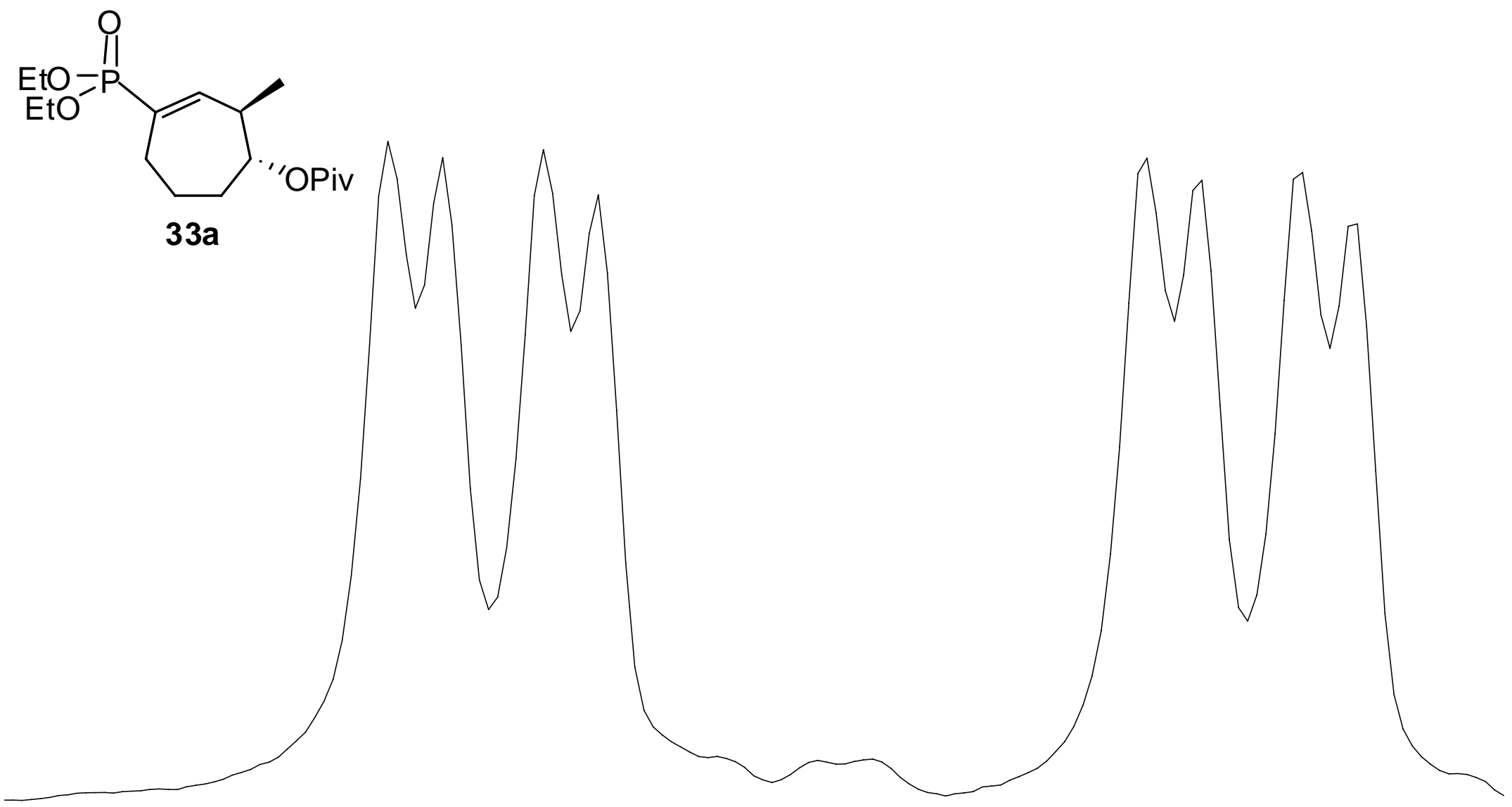

ppm

$$
6.600
$$

6.550

6.500 

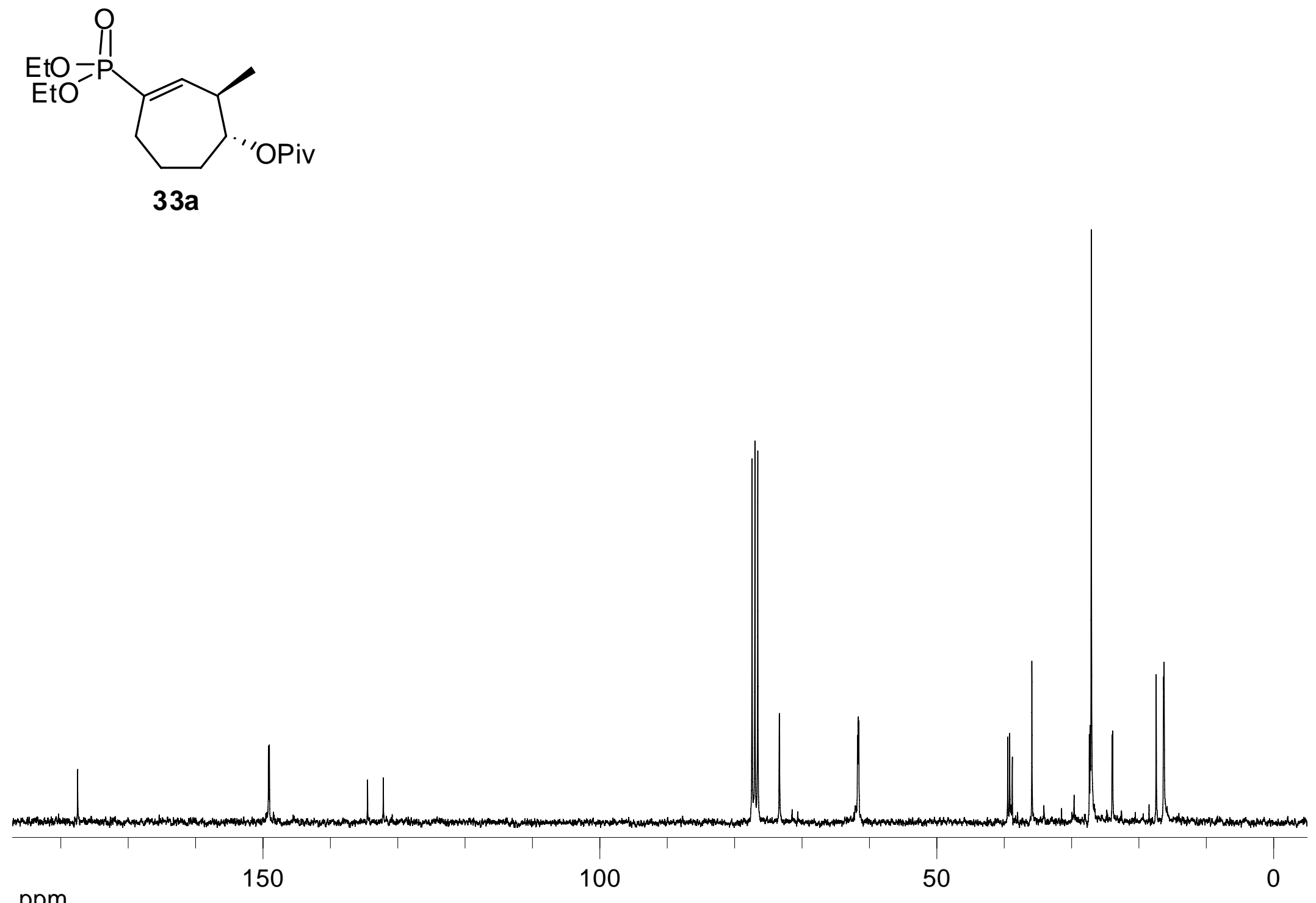

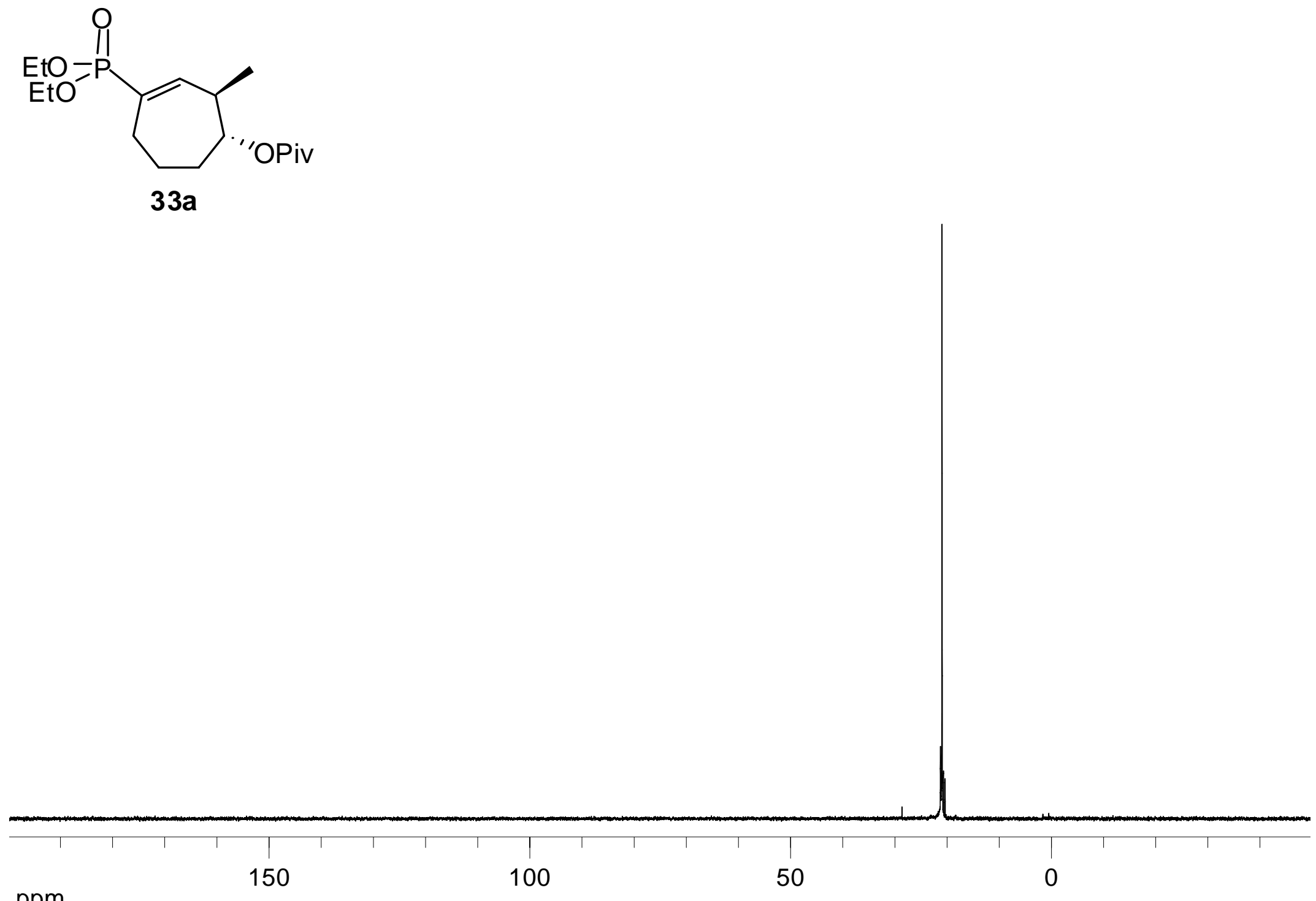

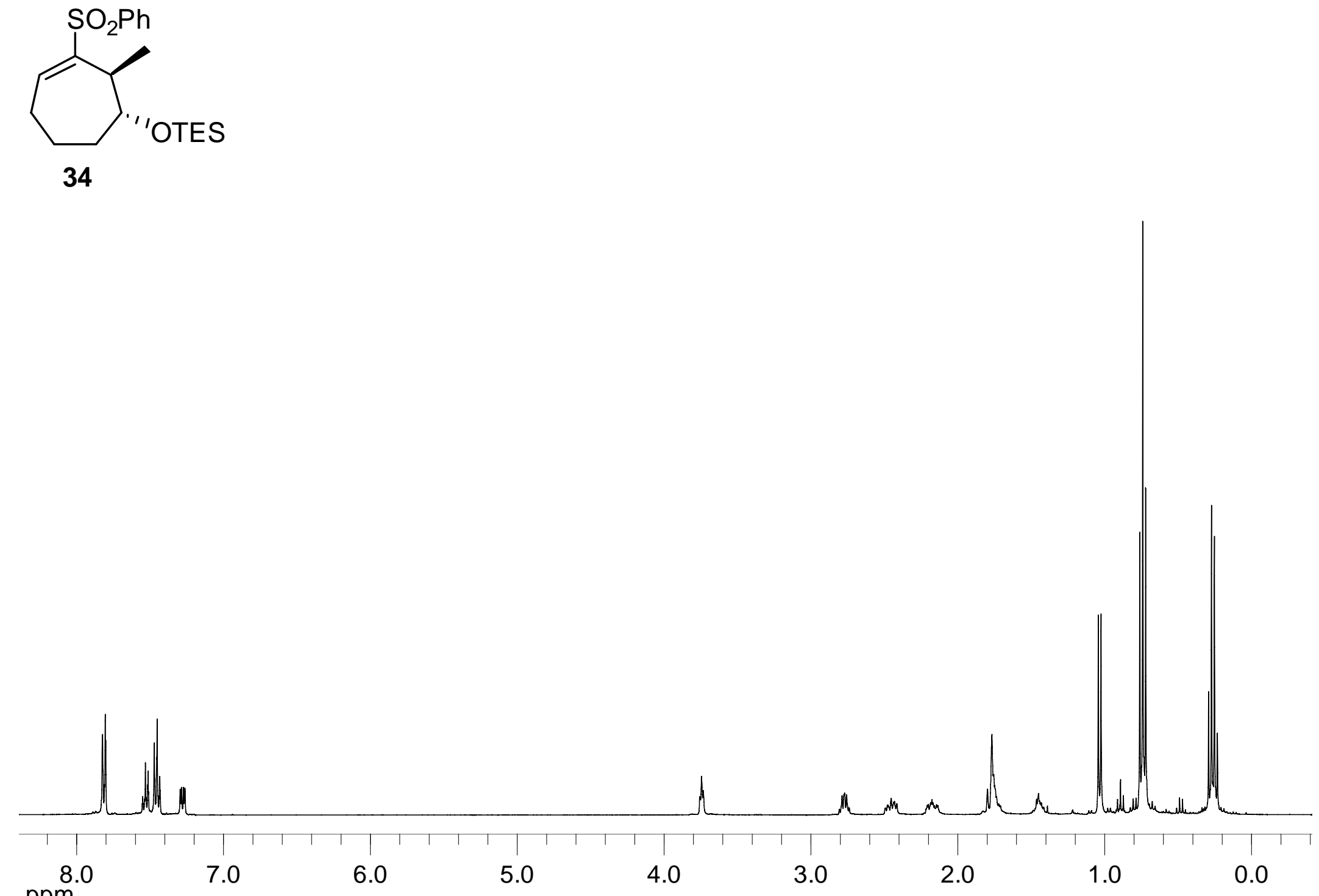


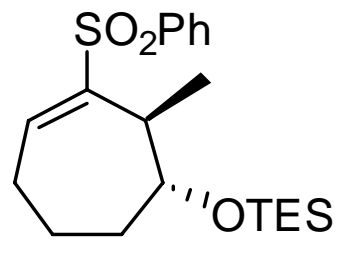

34

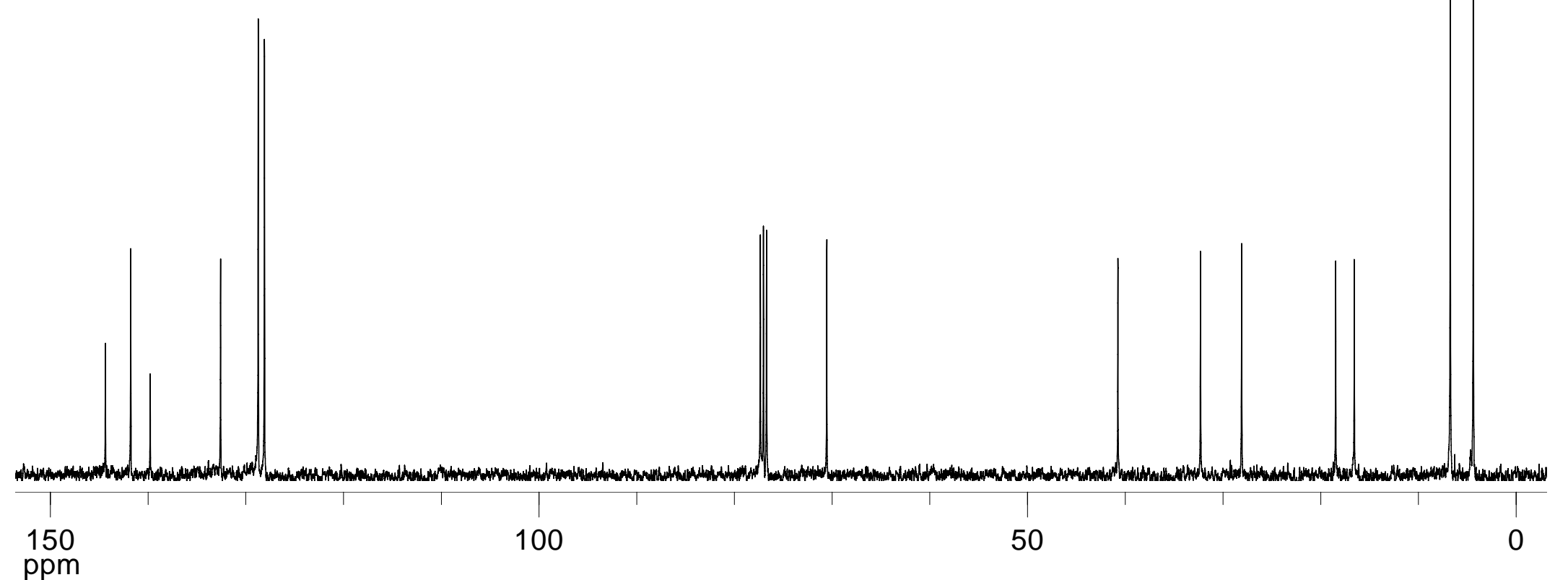



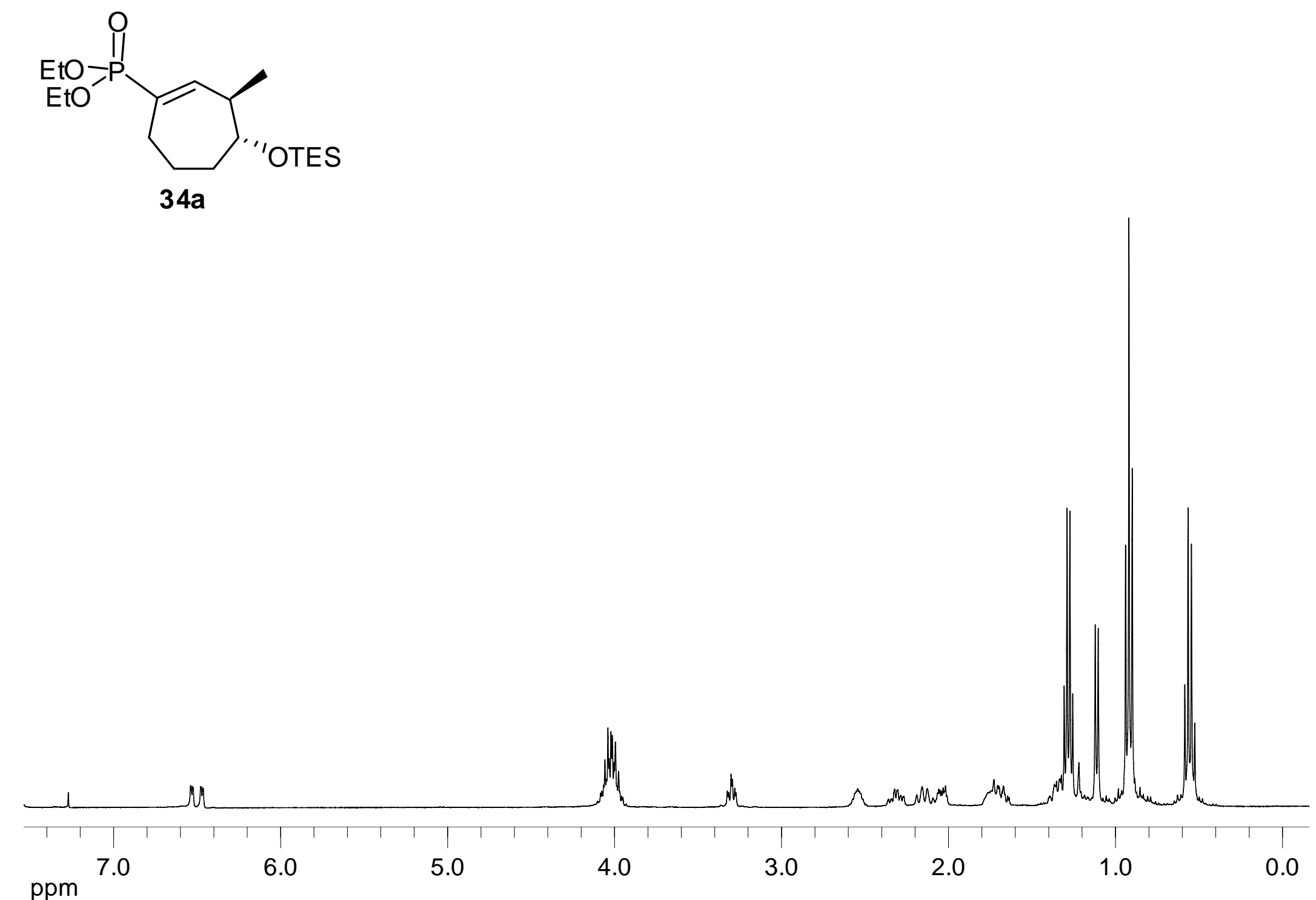

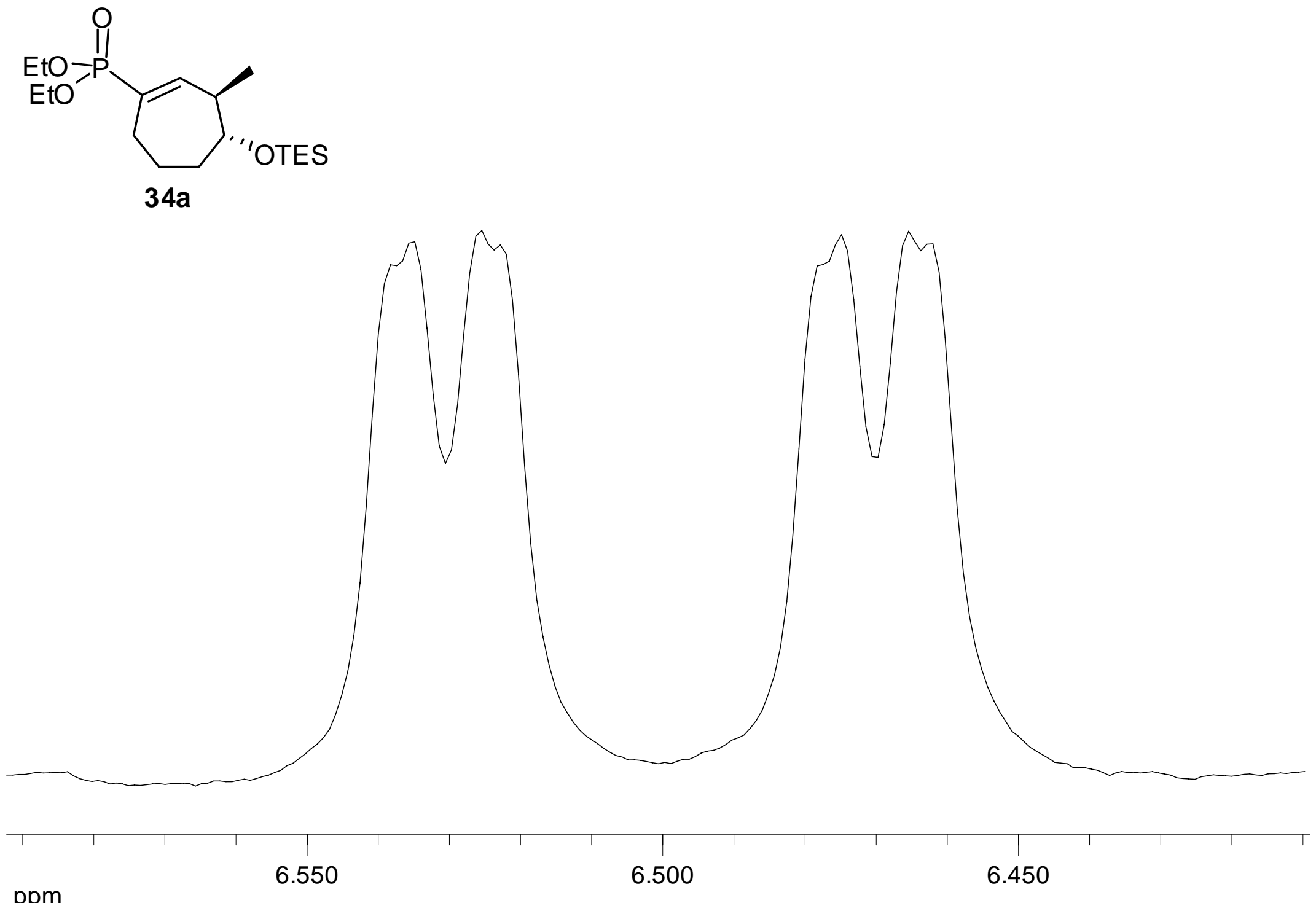

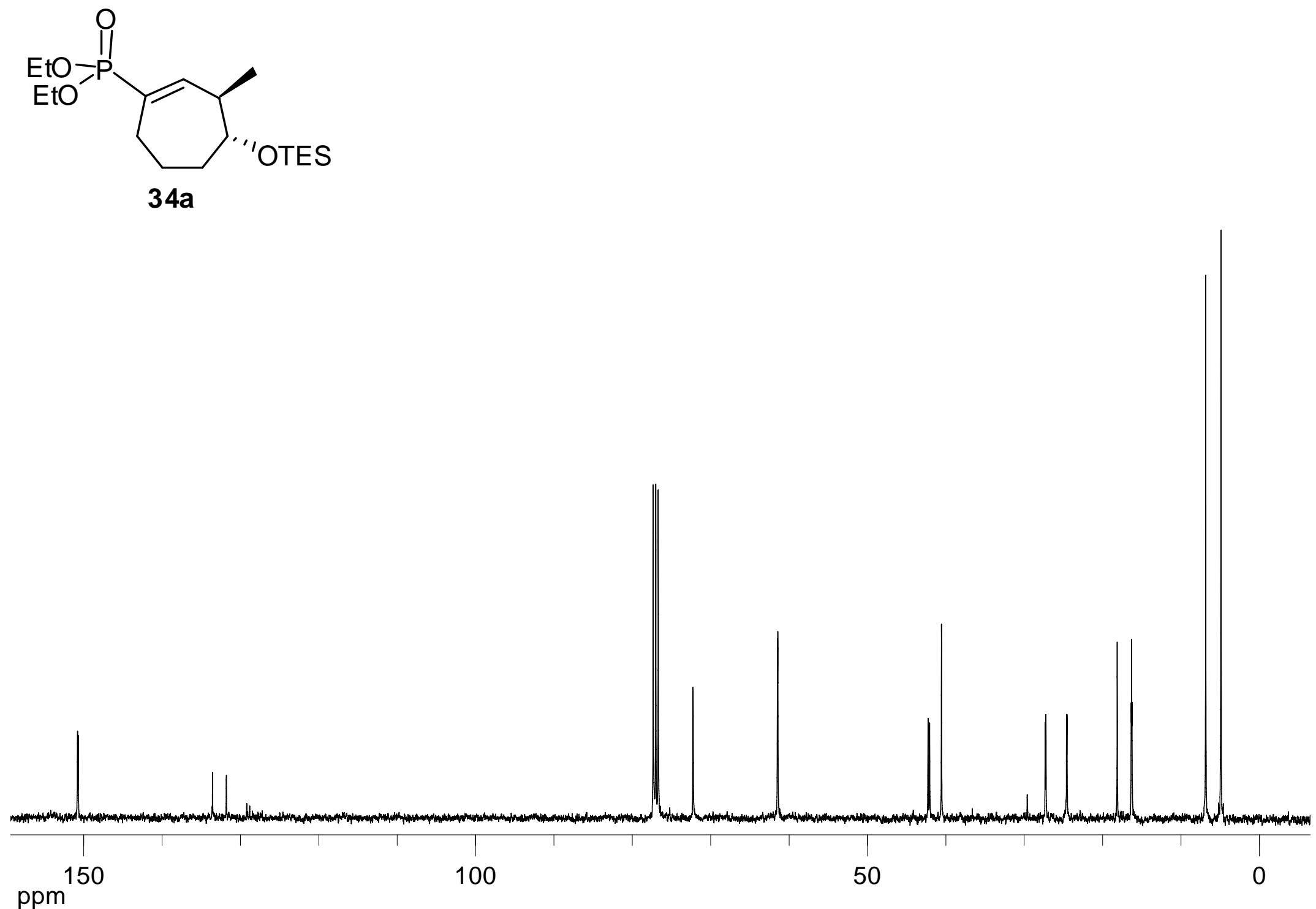

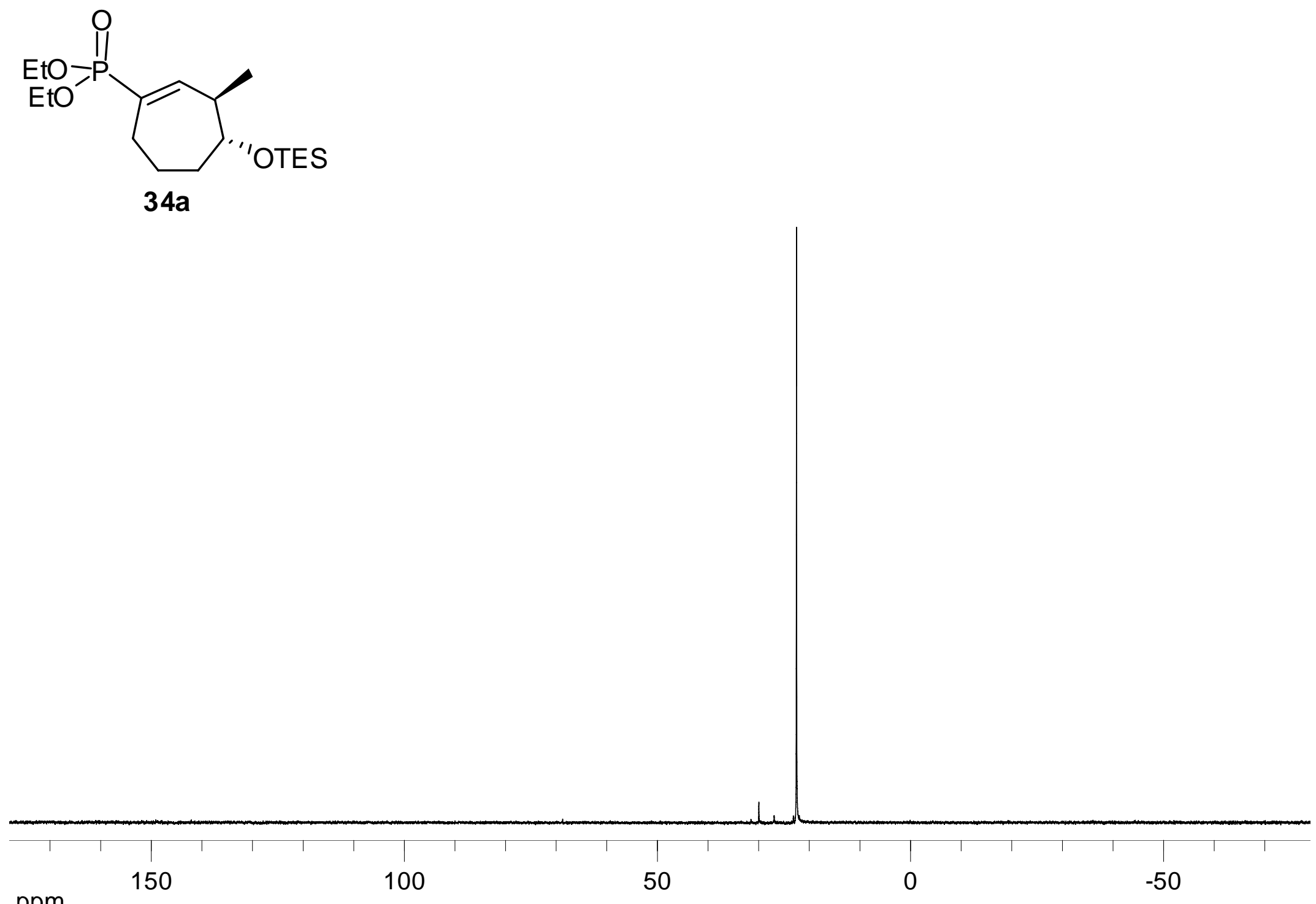

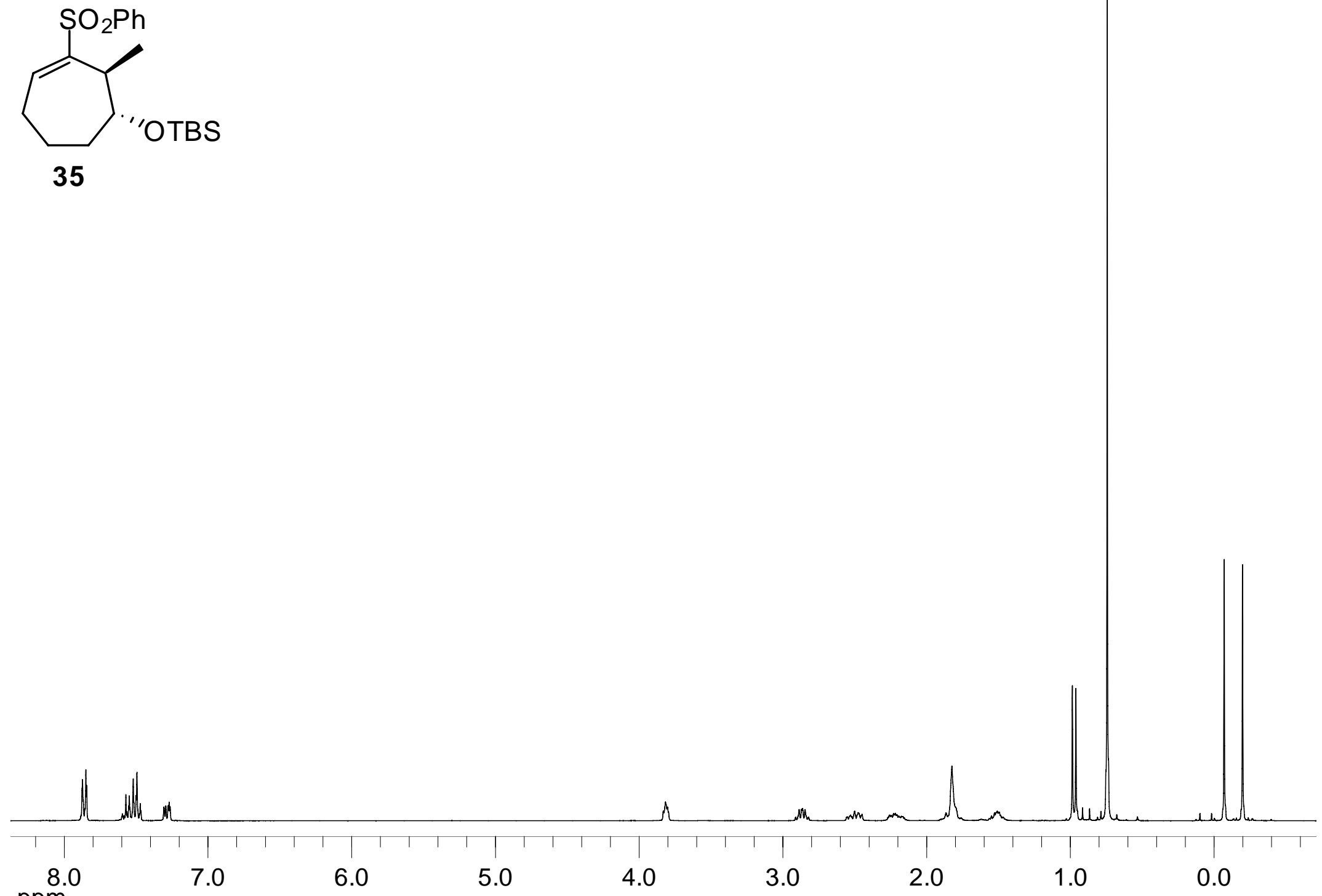


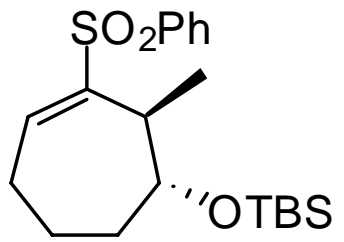

35

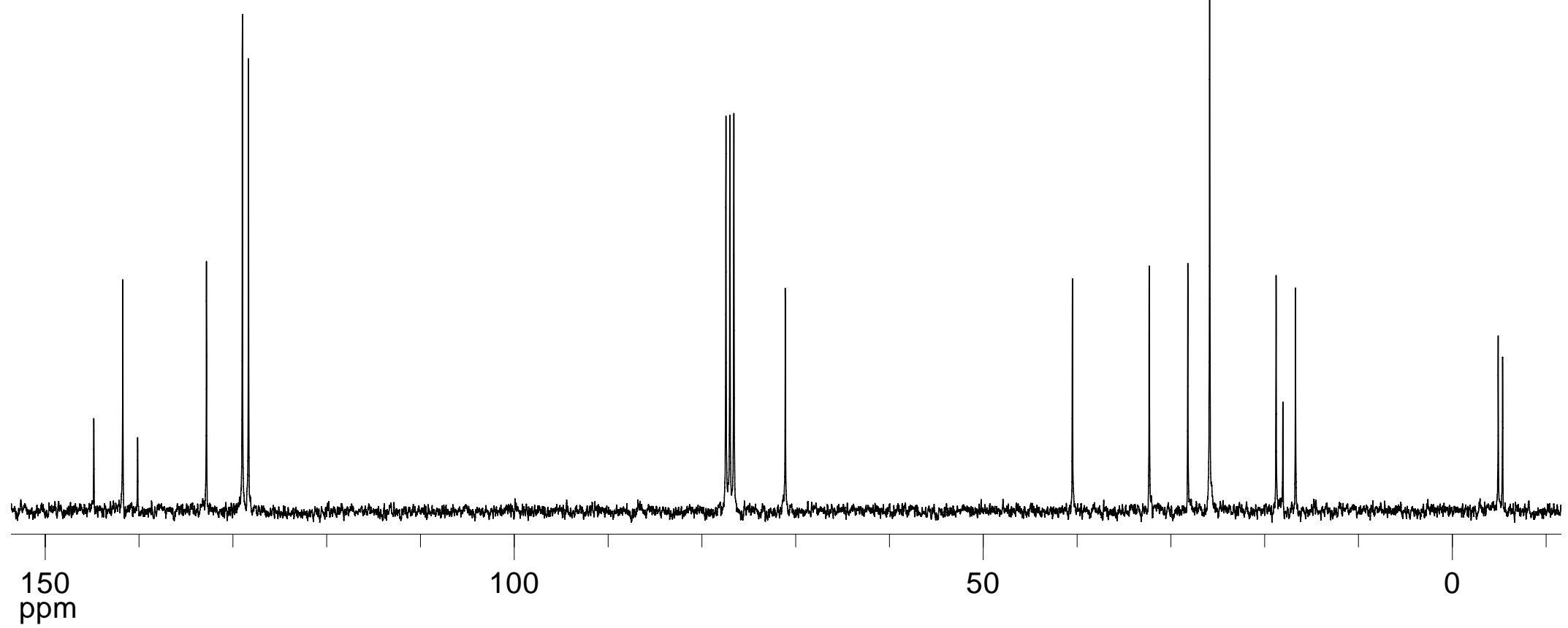




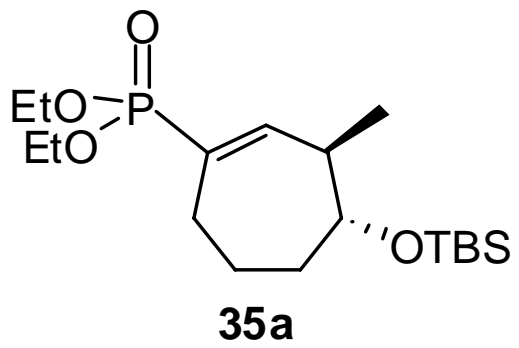



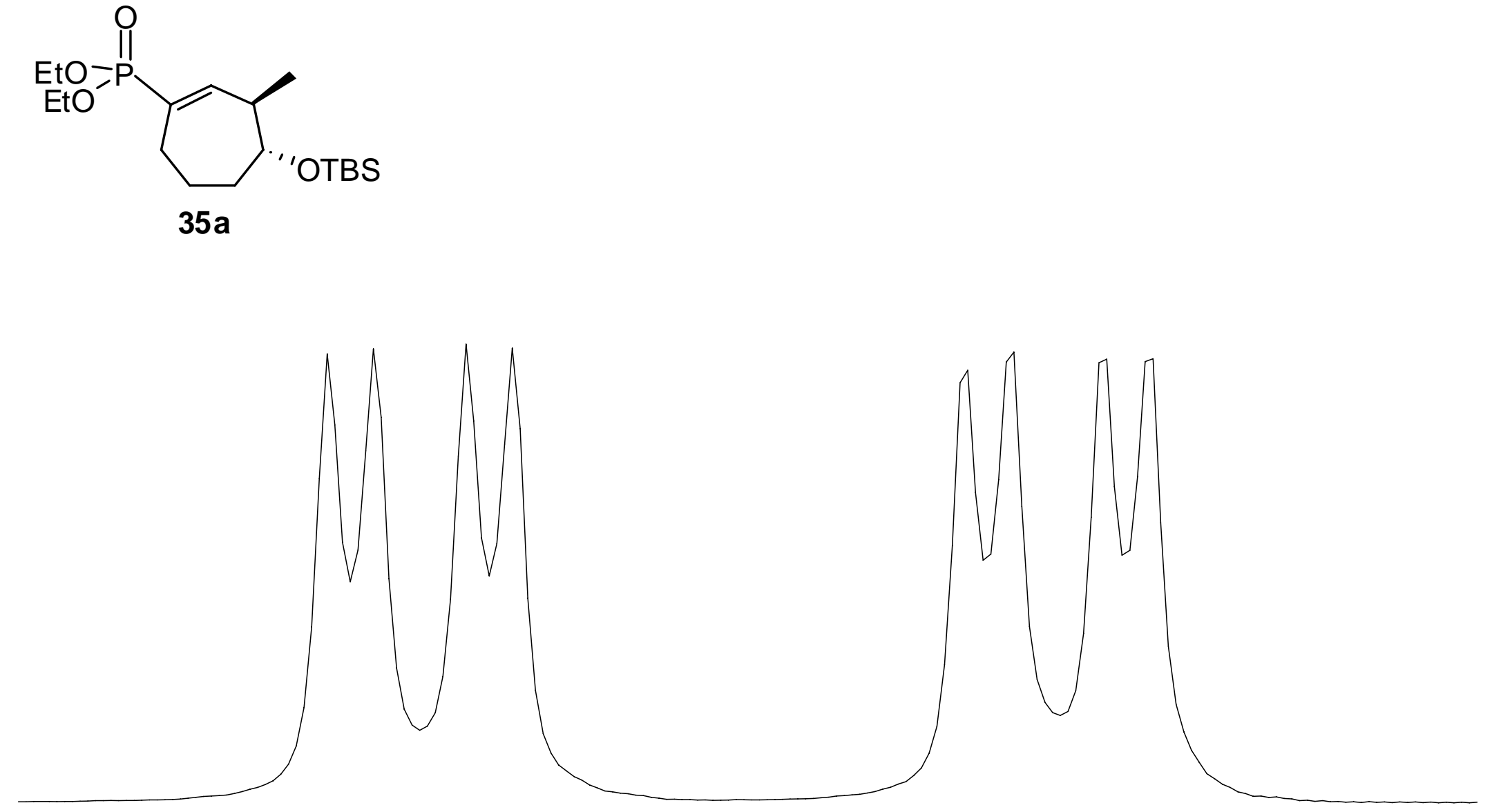

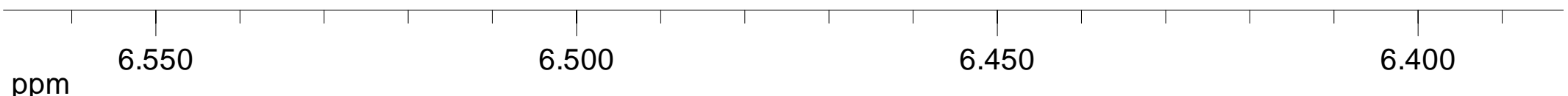




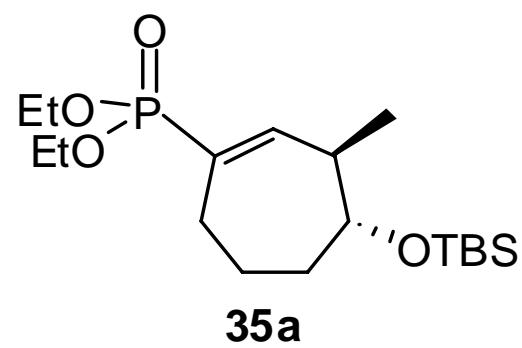

$35 a$

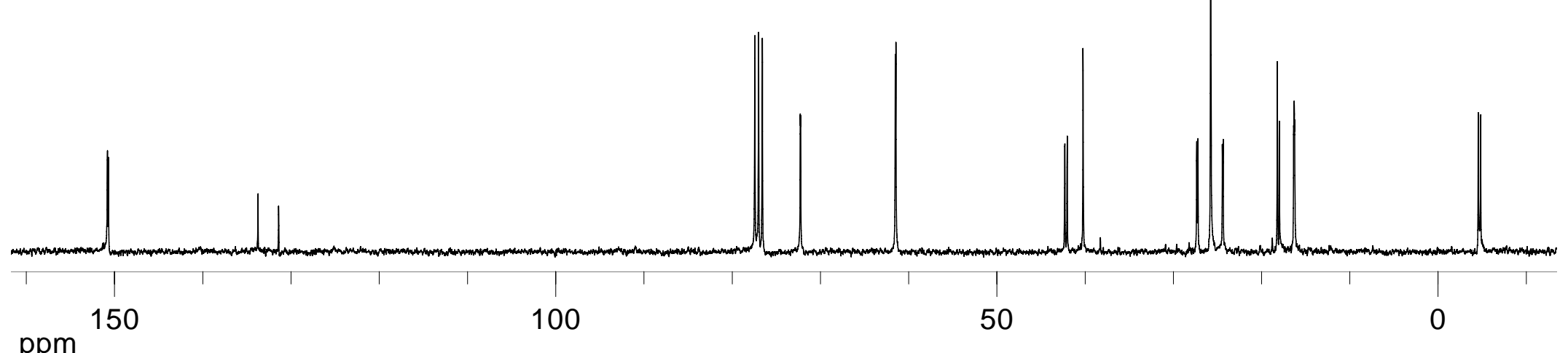




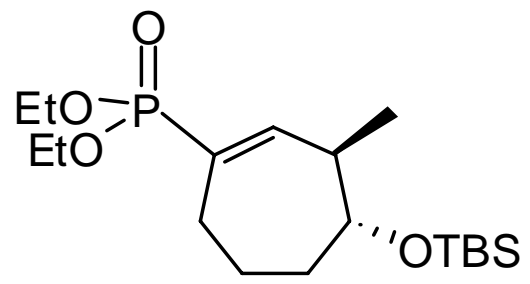

$35 a$

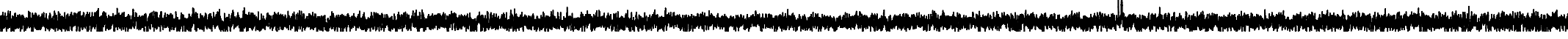

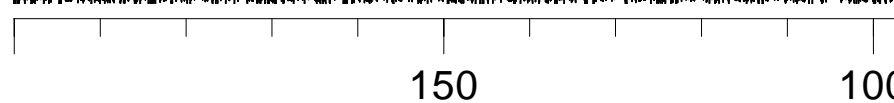

100

50

0

ppm 

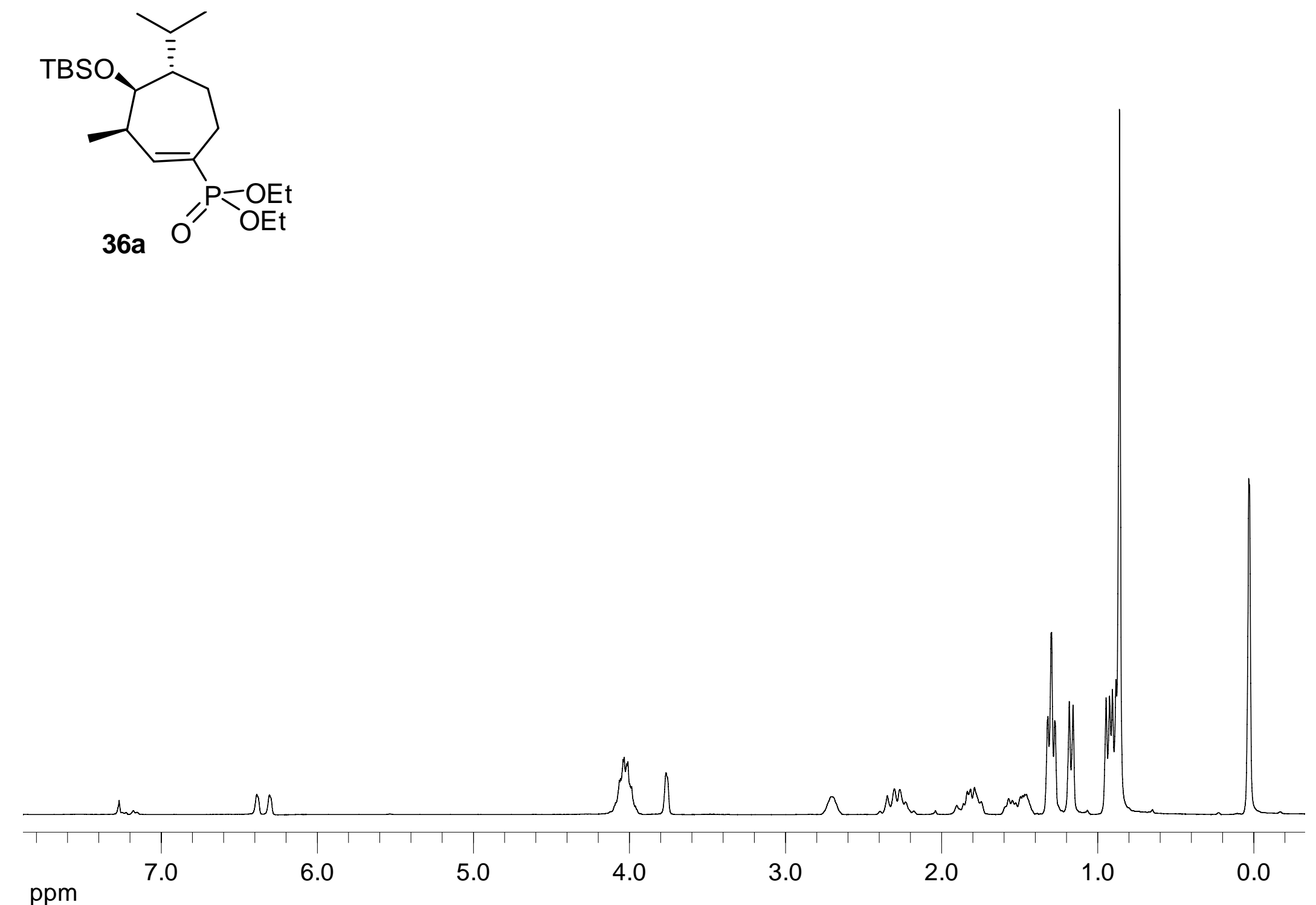


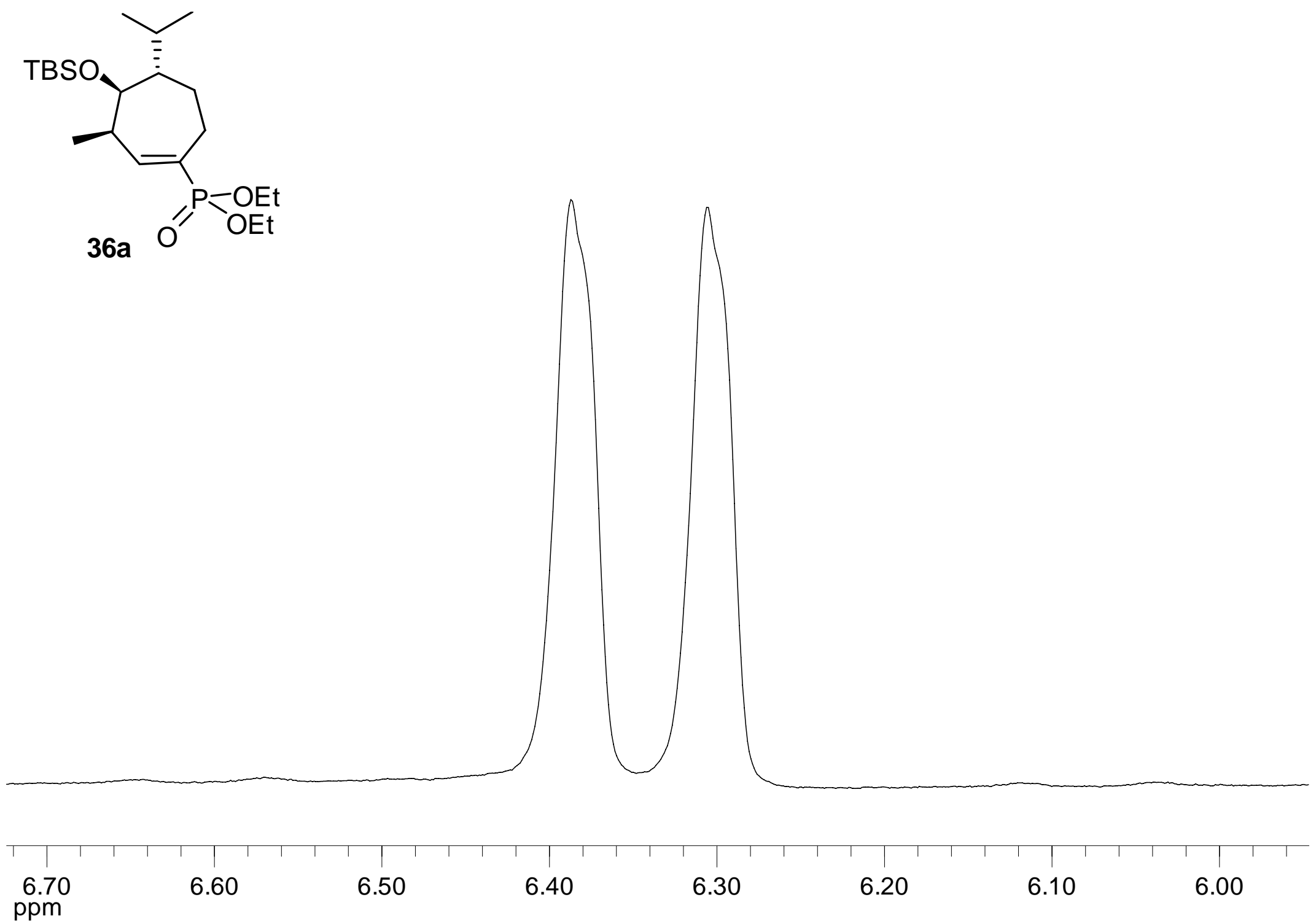



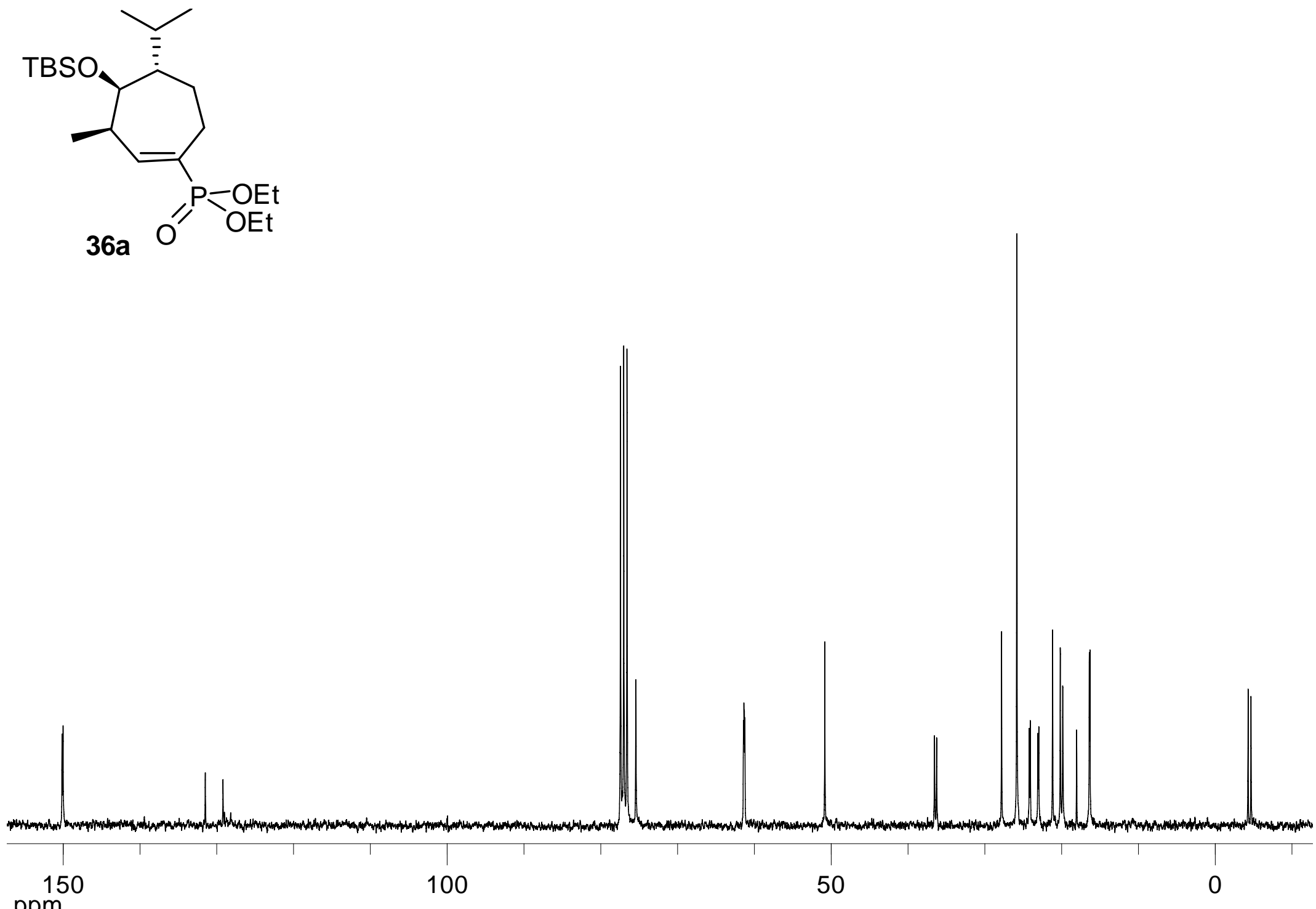
ppm 

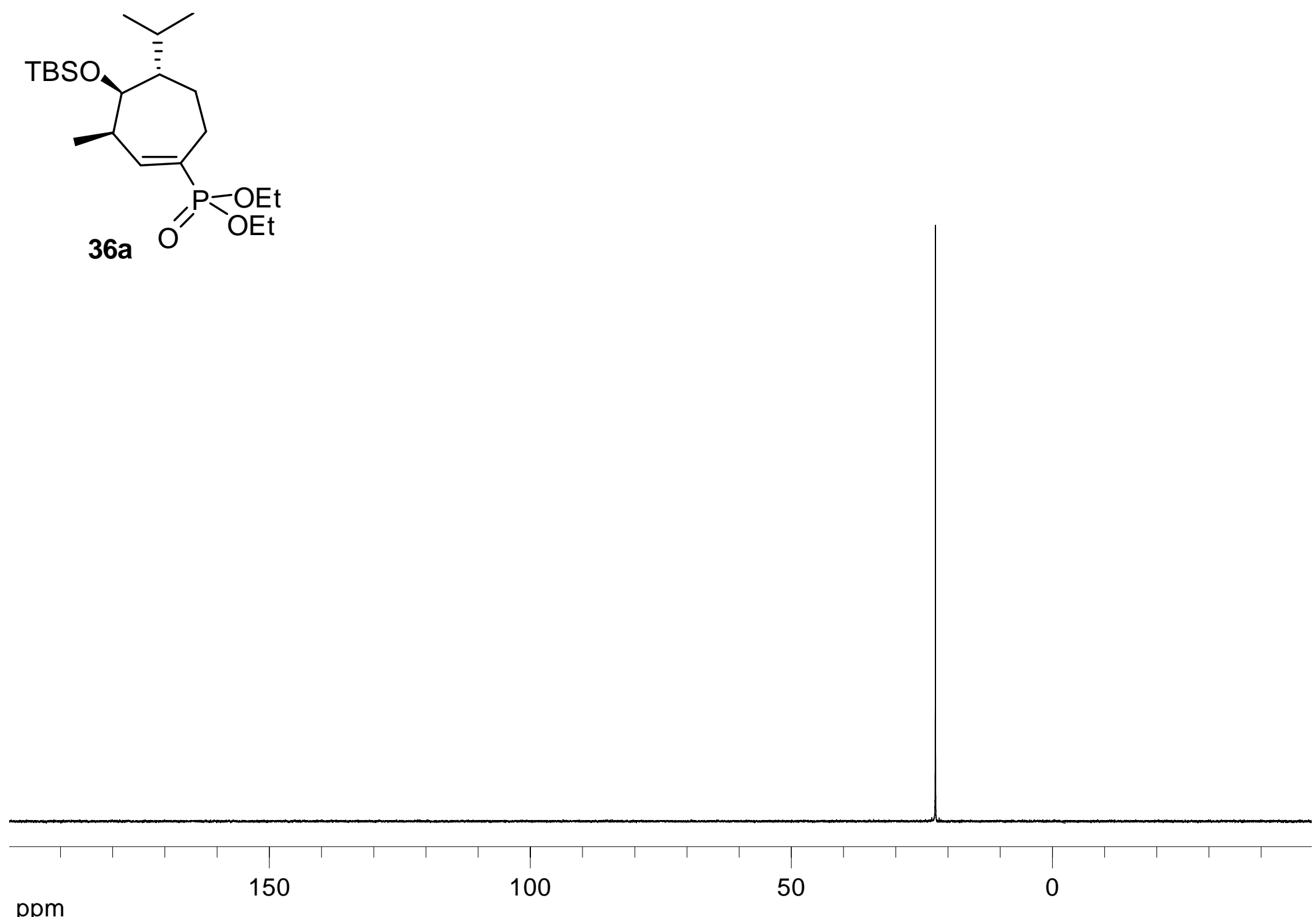

ppm 